\title{
Evolution of neutrino mass-mixing parameters in matter with non-standard interactions
}

\author{
Sanjib Kumar Agarwalla, ${ }^{a, b, c, 1}$ Sudipta Das, ${ }^{a, b, 2}$ Mehedi Masud ${ }^{a, d, 3}$ \\ and Pragyanprasu Swain ${ }^{a, b, 4}$
}

\author{
${ }^{a}$ Institute of Physics, \\ Sachivalaya Marg, Sainik School Post, Bhubaneswar 751005, India \\ ${ }^{b}$ Homi Bhabha National Institute, Training School Complex, \\ Anushakti Nagar, Mumbai 400094, India \\ ${ }^{c}$ International Centre for Theoretical Physics, \\ Strada Costiera 11, 34151 Trieste, Italy \\ ${ }^{d}$ Center for Theoretical Physics of the Universe, Institute for Basic Science (IBS), \\ Daejeon 34126, South Korea \\ E-mail: sanjib@iopb.res.in, sudipta.d@iopb.res.in, masud@ibs.re.kr, \\ pragyanprasu.s@iopb.res.in
}

ABSTRACT: We explore the role of matter effect in the evolution of neutrino oscillation parameters in the presence of lepton-flavor-conserving and lepton-flavor-violating neutralcurrent non-standard interactions (NSI) of the neutrino. We derive simple approximate analytical expressions showing the evolution of mass-mixing parameters in matter with energy in the presence of standard interactions (SI) and SI+NSI (considering both positive and negative values of real NSI parameters). We observe that only the NSI parameters in the $(2,3)$ block, namely $\varepsilon_{\mu \tau}$ and $(\gamma-\beta) \equiv\left(\varepsilon_{\tau \tau}-\varepsilon_{\mu \mu}\right)$ affect the modification of $\theta_{23}$. Though all the NSI parameters influence the evolution of $\theta_{13}, \varepsilon_{e \mu}$ and $\varepsilon_{e \tau}$ show a stronger impact at the energies relevant for DUNE. The solar mixing angle $\theta_{12}$ quickly approaches to $\sim 90^{\circ}$ with increasing energy in both SI and SI+NSI cases. The change in $\Delta m_{21, m}^{2}$ is quite significant as compared to $\Delta m_{31, m}^{2}$ both in SI and SI+NSI frameworks for the energies relevant for DUNE baseline. Flipping the signs of the NSI parameters alters the way in which mass-mixing parameters run with energy. We demonstrate the utility of our approach in addressing several important features related to neutrino oscillation such as: a) unraveling interesting degeneracies between $\theta_{23}$ and NSI parameters, b) estimating the

\footnotetext{
${ }^{1}$ https://orcid.org/0000-0002-9714-8866.

${ }^{2}$ https://orcid.org/0000-0002-5508-7751.

${ }^{3}$ https://orcid.org/0000-0002-7014-3520.

${ }^{4}$ https://orcid.org/0000-0003-3008-480X.
} 
resonance energy in presence of NSI when $\theta_{13}$ in matter becomes maximal, c) figuring out the required baselines and energies to have maximal matter effect in $\nu_{\mu} \rightarrow \nu_{e}$ transition in the presence of different NSI parameters, and d) studying the impact of NSI parameters $\varepsilon_{\mu \tau}$ and $(\gamma-\beta)$ on the $\nu_{\mu} \rightarrow \nu_{\mu}$ survival probability.

Keywords: Beyond Standard Model, Neutrino Physics

ArXiv EPrint: 2103.13431 


\section{Contents}

1 Introduction and motivation 1

2 Theoretical formalism of NSI 4

3 Diagonalization of the effective Hamiltonian in the presence of NSI 6

4 Evolution of mixing angles in the presence of NSI 9

4.1 Evolution of $\theta_{23}^{m} \quad 9$

4.2 Evolution of $\theta_{13}^{m} \quad 13$

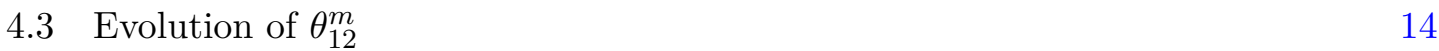

5 Evolution of mass-squared differences in the presence of NSI 16

$6 \quad \theta_{13}$-resonance in the presence of NSI $\quad 19$

7 Impact of NSI in $\nu_{\mu}-\nu_{e}$ appearance channel 20

8 Impact of NSI in $\nu_{\mu} \rightarrow \nu_{\mu}$ disappearance channel $\quad 27$

9 Summary and concluding remarks $\quad 30$

A Comparison between approximate analytical expressions and exact numerical calculations at the probability level 32

B Evolution of mass-mixing parameters with non-zero $\delta_{\mathrm{CP}}$

C Calculation of the oscillation probabilities for neutrinos passing $\begin{array}{ll}\text { through various layers inside the Earth } & 37\end{array}$

\section{Introduction and motivation}

The phenomenon of three-flavor neutrino oscillation is governed by the six fundamental mass-mixing parameters [1]: a) three mixing angles: $\theta_{12}, \theta_{13}, \theta_{23}$, b) two independent masssquared differences: $\Delta m_{21}^{2} \equiv m_{2}^{2}-m_{1}^{2}, \Delta m_{31}^{2} \equiv m_{3}^{2}-m_{1}^{2}$, and c) one Dirac CP phase $\delta_{\mathrm{CP}}$. After the discovery of neutrino oscillation at the Super-Kamiokande (Super-K) experiment in 1998 [2], fantastic data from the world-class accelerator, atmospheric, reactor, and solar neutrino experiments are pouring in day-by-day to commence the era of precision neutrino measurement science [3-6], which will certainly provide crucial insights on the possible origin of neutrino mass and mixing [7-9]. 
Marvelous data from several ongoing experiments such as Super-K [10], IceCubeDeepCore [11], ANTARES [12], Daya Bay [13], RENO [14], Tokai to Kamioka (T2K) [15, 16], and NuMI Off-axis $\nu_{e}$ Appearance $(\mathrm{NO} \nu \mathrm{A})[17]$ have been improving our knowledge about the neutrino oscillation parameters beyond expectations. Because of this fascinating progress, we have been able to build a robust, simple, three-flavor neutrino oscillation paradigm which successfully accommodate most of the data [3-6].

Future high-precision neutrino oscillation experiments such as the Deep Underground Neutrino Experiment (DUNE) [18, 19], Tokai to Hyper-Kamiokande (T2HK) [20], Tokai to Hyper-Kamiokande with a second detector in Korea (T2HKK) [21], European Spallation Source $\nu$ Super Beam (ESS $\nu$ SB) [22], India-based Neutrino Observatory (INO) [23-25], Jiangmen Underground Neutrino Observatory (JUNO) [26], and THEIA [27] aim to determine the oscillation parameters with a precision around a few \%. Therefore, these next generation experiments are potentially sensitive to various sub-leading beyond the Standard Model (BSM) effects [28, 29]. One such interesting BSM scenario is non-standard neutrino interactions (NSI) [30-46] which is the main focus of this paper.

Analytical understanding of neutrino oscillation probabilities over a wide range of energies and baselines becomes non-trivial in the presence of standard interactions (SI). ${ }^{1}$ Now, on top of that if NSI exist in Nature then the task becomes even more complex. Assuming the line-averaged constant Earth matter density for a given baseline, several authors have derived approximate analytical expressions for the neutrino oscillation probabilities ${ }^{2}$ in the presence of SI [50-61] and SI+NSI [62-70].

To obtain a better understanding of the neutrino oscillation probabilities as functions of baseline $L$ and/or neutrino energy $E$ in the presence of SI or SI+NSI, it is quite important in the first place to have a clear knowledge on how various mixing angles and mass-squared differences get modified in matter with energy for a given baseline. Simple approximate analytical expressions showing the evolution of mass-mixing parameters in matter with energy in the presence of SI and SI+NSI allow us to address several important features that show up in neutrino oscillation in a more general and transparent fashion. This simple and more intuitive way to understand the neutrino oscillation phenomena will likely pave a way to disentangle the various non-trivial correlations/degeneracies that may be present among the various oscillation and NSI parameters. This paper addresses several pressing issues along this direction.

There exist several studies in the literature investigating how the presence of SI and NSI affect the evolution of effective neutrino oscillation parameters (the mixing angles, mass-squared differences, and CP-violating phase) in matter with energy, and eventually how they modify the oscillation probabilities [55, 57, 59-61, 68-78]. In refs. [71, 72, 74, 75],

\footnotetext{
${ }^{1}$ They appear into the picture due to the Standard Model (SM) $W$-exchange interactions between the ambient matter electrons and the propagating electron neutrinos, which is popularly known as the 'MSW effect' $[30,47,48]$.

${ }^{2}$ In ref. [49], the authors performed a detailed comparative study between different expansions for neutrino oscillation probabilities in the presence of SI in matter. They also studied the accuracy and computational efficiency of several exact and approximate expressions for neutrino oscillation probabilities in the context of long-baseline (LBL) experiments.
} 
the authors diagonalize analytically the three-flavor propagation Hamiltonian in constantdensity matter to obtain the exact expressions for the modified mass-mixing parameters in the presence of SI. The authors in ref. [73] make use of the Cayley-Hamilton approach with a plane wave approximation to derive the expressions for the modified mass-mixing parameters without performing the actual diagonalization of the Hamiltonian. They also briefly discuss how these oscillation parameters get modified with the strength of SI. In ref. [55], the author diagonalizes the neutrino propagation Hamiltonian in the presence of SI by applying successive rotations and obtain the expressions for the modified mass-mixing parameters. In ref. [75], the authors make use of the relations between the Jarlskog invariants in vacuum and matter (Naumov-Harrison-Scott identities [79-81]) to derive the expressions for modified mass-mixing parameters in the presence of SI in constant-density matter. In ref. [69], the authors adopt a perturbative approach towards the SI and NSI effects and discuss the possible modifications of the mass-mixing parameters. In refs. [76-78], the authors apply the techniques of renormalization-group equations (RGEs) to understand how the neutrino mass-mixing parameters evolve in the presence of SI in constant-density matter. In most of these studies, the authors extract the expressions for modified mass-mixing parameters in order to obtain approximate analytical expressions for the neutrino oscillation probabilities. Using the Jacobi method [82], the authors in ref. [59] show that the matter effect on neutrino oscillation due to SI could be assimilated into the evolution of the effective mixing angles $\theta_{12}$ and $\theta_{13}$, and the effective mass-squared differences in matter as functions of the Wolfenstein matter term $2 \sqrt{2} G_{F} N_{e} E$, while the effective values of $\theta_{23}$ and $\delta_{\mathrm{CP}}$ remain unaltered. Here, $G_{F}$ is the Fermi muon decay constant, $N_{e}$ is the ambient electron number density, and $E$ is the energy of the neutrino. They obtain the approximate neutrino oscillation probabilities by simply replacing the mass-mixing parameters in the expressions for the probabilities in vacuum with their modified counterparts. Similar approach is adopted by the authors in ref. [70] to show the evolution of mass-mixing parameters in the presence of lepton-flavor-conserving, non-universal NSI of the neutrino.

In the present work, we perform successive rotations to almost diagonalize the propagation Hamiltonian in the presence of SI and SI+NSI and derive simple approximate analytical expression for the effective mass-mixing parameters in constant-density matter. While deriving our expressions, we retain the terms of all orders in $\sin \theta_{13}$ and $\alpha$ (the ratio of solar and atmospheric mass-squared differences, $\left.\Delta m_{21}^{2} / \Delta m_{31}^{2}\right)$ which are quite important in light of the large value of $\theta_{13}$. In our study, we also entertain all possible allowed values of $\theta_{23}$ in vacuum. As far as NSI are concerned, we consider all possible lepton-flavor-conserving and lepton-flavor-violating neutral-current (NC) NSI at-a-time in our analysis which affect the propagation of neutrino in matter. We discuss many salient features of the evolution of oscillation parameters with energy for some benchmark choices of baseline and study in detail how these mass-mixing parameters get affected by various combinations of NSI parameters. Our simple analytical expressions enable us to explore the possible degeneracies between $\theta_{23}$ (which still has large uncertainty) and NSI parameters for a given choice of neutrino mass ordering in a simple manner. For the first time, we show how the famous MSW-resonance condition $\left(\theta_{13}\right.$ in matter becomes $\left.45^{\circ}\right)[30,47,48,83]$ gets altered in the presence of NC-NSI. We demonstrate how the simple approximate analytical expressions 
for the modified oscillation parameters in matter help us to estimate the baselines and energies for which we have the maximal matter effect in $\nu_{\mu} \rightarrow \nu_{e}$ oscillation channel in the presence of various NSI parameters. For simplicity, we perform our calculations in a $\mathrm{CP}$-conserving scenario where the standard Dirac CP phase $\delta_{\mathrm{CP}}$ and the phases associated with the lepton-flavor-violating NSI parameters are assumed to be zero. We consider both positive and negative values of real NSI parameters in our analysis.

We plan this paper in the following fashion. We start section 2 with a brief discussion on the theoretical formalism of NSI. This is followed by a short summary of the existing bounds on the NC-NSI. In section 3, we describe our method of approximately diagonalizing the effective neutrino propagation Hamiltonian in the presence of all possible NC-NSI in constant-density matter. Subsequently, we derive the expressions for the modified massmixing parameters. In section 4 , we study the evolution of $\theta_{23}, \theta_{13}$, and $\theta_{12}$ in matter with energy in detail for some benchmark choices of baseline and analyze the role of various NSI parameters on their evolution. We illustrate the impact of SI and various NSI parameters on the variation of two modified mass-squared differences in section 5 . In section 6 , using the expressions for modified mass-mixing parameters, we estimate for the first time a simple and compact expression for the $\theta_{13}$-resonance energy in the presence of all possible NC-NSI parameters and identify the NSI parameters that significantly affect the $\theta_{13}$-resonance energy. We devote section 7 to exhibit the utility of our approach in determining the baselines and energies for which we can achieve the maximal matter effect in $\nu_{\mu} \rightarrow \nu_{e}$ transition in the presence of various NSI parameters. Section 8 describes how the NSI parameters in the $(2,3)$ block affect $\nu_{\mu} \rightarrow \nu_{\mu}$ disappearance channel. Finally, we summarize and draw our conclusions in section 9. In appendix A, we perform a comparison between various oscillation probabilities obtained using our approximate analytical expressions and exact numerical calculations. In appendix B, we derive approximate analytical expressions showing the evolution of the oscillation parameters in matter in the presence of SI and SI+NSI considering non-zero values of Dirac CP phase $\delta_{\mathrm{CP}}$. We also compare $\nu_{\mu} \rightarrow \nu_{e}$ oscillation probabilities obtained using our approximate analytical expressions and full numerical results from the GLoBES software in the presence of NC-NSI parameters $\left(\varepsilon_{e \mu}\right.$, $\left.\varepsilon_{e \tau}\right)$ and non-zero values of $\delta_{\mathrm{CP}}$. In appendix $\mathrm{C}$, we discuss in detail how we estimate various oscillation probabilities considering a four-layered profile of Earth.

\section{Theoretical formalism of NSI}

NSI which arise naturally in most of the neutrino mass models can be of charged-current (CC) or neutral-current (NC) in nature. Both of them can be described with a dimension-six operator in the four-fermion effective Lagrangian [30, 34, 40],

$$
\begin{aligned}
& \mathcal{L}_{\mathrm{NC}-\mathrm{NSI}}=-2 \sqrt{2} G_{F} \sum_{\alpha, \beta, f, C} \varepsilon_{\alpha \beta}^{f C}\left(\bar{\nu}_{\alpha} \gamma^{\mu} P_{L} \nu_{\beta}\right)\left(\bar{f} \gamma_{\mu} P_{C} f\right), \\
& \mathcal{L}_{\mathrm{CC}-\mathrm{NSI}}=-2 \sqrt{2} G_{F} \sum_{\alpha, \beta, f^{\prime}, f, C} \varepsilon_{\alpha \beta}^{f f^{\prime} C}\left(\bar{\nu}_{\alpha} \gamma^{\mu} P_{L} l_{\beta}\right)\left(\bar{f}^{\prime} \gamma_{\mu} P_{C} f\right),
\end{aligned}
$$

where, $P_{C}$ indicates the chiral projection operators $P_{L}$ or $P_{R}$. The dimensionless coefficients $\varepsilon_{\alpha \beta}^{f C}$ in eq. (2.1) denote the strength of NC-NSI between the leptons of flavors $\alpha$ and $\beta$ 
$(\alpha, \beta=e, \mu, \tau)$, and the first generation fermions $f \in\{e, u, d\}$. In eq. (2.2), the dimensionless coefficients $\varepsilon_{\alpha \beta}^{f f^{\prime} C}$ indicate the strength of CC-NSI between the leptons of $\alpha$ and $\beta$ flavors $(\alpha, \beta=e, \mu, \tau)$, and the first generation fermions $f \neq f^{\prime} \in\{u, d\}$. The hermiticity of these interactions imposes the following conditions:

$$
\varepsilon_{\alpha \beta}^{f C}=\left(\varepsilon_{\beta \alpha}^{f C}\right)^{*}, \quad \varepsilon_{\alpha \beta}^{f f^{\prime} C}=\left(\varepsilon_{\beta \alpha}^{f f^{\prime} C}\right)^{*} .
$$

The CC-NSI modify the production and detection of neutrinos and may also lead to charged-lepton flavor violation. The NC-NSI, on the other hand, affect the propagation of neutrinos. Since the coupling strength $\varepsilon_{\alpha \beta}^{f C}$ enters into the Lagrangian only through vector coupling, we can write $\varepsilon_{\alpha \beta}^{f}=\varepsilon_{\alpha \beta}^{f L}+\varepsilon_{\alpha \beta}^{f R}$. It is worthwhile to mention here that models employing scalar mediators [84] or other spin structures [85] are also available in the literature. Beyond a simplified model approach, many UV complete models for NSI have also been explored (see, for instance, [86-90]). For a recent comprehensive review of the NSI, see [43]. Using eqs. (2.1) and (2.2) and the well-known relation $G_{F} / \sqrt{2} \simeq g_{W}^{2} / 8 m_{W}^{2}$, it can be shown that the effective NSI parameters $(\varepsilon)$ are proportional to $m_{W}^{2} / m_{X}^{2}[62,65,91]$, where $g_{W}$ is the coupling constant of the weak interaction, $m_{W}$ is the $\mathrm{W}$ boson mass $(\simeq 80 \mathrm{GeV} \sim 0.1 \mathrm{TeV})$, and $m_{X}$ is the mass scale where NSI are generated. Thus it can easily be observed that for $m_{X} \sim 1 \mathrm{TeV}$, the NSI parameters are of the order of $10^{-2}$.

In the present work, we concentrate on the NC-NSI which appear during neutrino propagation through matter. Here, the effective NSI parameter can be written in the following fashion

$$
\varepsilon_{\alpha \beta} \equiv \sum_{f=e, u, d} \varepsilon_{\alpha \beta}^{f} \frac{N_{f}}{N_{e}} \equiv \sum_{f=e, u, d}\left(\varepsilon_{\alpha \beta}^{f L}+\varepsilon_{\alpha \beta}^{f R}\right) \frac{N_{f}}{N_{e}} .
$$

Here, $N_{f}$ is the first generation $(e, u, d)$ fermion number density in the ambient medium.

The effective Hamiltonian for neutrinos propagating in matter in presence of all the lepton-flavor-conserving and lepton-flavor-violating NC-NSI can be written as

$$
H_{f}=\frac{1}{2 E}\left[U\left(\begin{array}{ccc}
0 & 0 & 0 \\
0 & \Delta m_{21}^{2} & 0 \\
0 & 0 & \Delta m_{31}^{2}
\end{array}\right) U^{\dagger}+2 E V_{C C}\left(\begin{array}{ccc}
1+\varepsilon_{e e} & \varepsilon_{e \mu} & \varepsilon_{e \tau} \\
\varepsilon_{e \mu}^{*} & \varepsilon_{\mu \mu} & \varepsilon_{\mu \tau} \\
\varepsilon_{e \tau}^{*} & \varepsilon_{\mu \tau}^{*} & \varepsilon_{\tau \tau}
\end{array}\right)\right],
$$

where, $\Delta m_{21}^{2}\left(\equiv m_{2}^{2}-m_{1}^{2}\right)$ and $\Delta m_{31}^{2}\left(\equiv m_{3}^{2}-m_{1}^{2}\right)$ are the solar and atmospheric masssquared differences, respectively. $U$ is the $3 \times 3$ unitary Pontecorvo-Maki-Nakagawa-Sakata (PMNS) matrix in vacuum [92-94], which can be parametrized using the three mixing angles: $\theta_{12}, \theta_{23}, \theta_{13}$, and one Dirac-type CP phase $\delta_{\mathrm{CP}}$ (ignoring Majorana phases) in the following fashion

$$
U=R_{23}\left(\theta_{23}, 0\right) R_{13}\left(\theta_{13}, \delta_{\mathrm{CP}}\right) R_{12}\left(\theta_{12}, 0\right) .
$$

In eq. (2.5), $V_{C C}$ is the standard $W$-exchange interaction potential in matter which can be expressed as

$$
V_{C C}=\sqrt{2} G_{F} N_{e} \approx 7.6 \times Y_{e} \times 10^{-14}\left[\frac{\rho}{\mathrm{g} / \mathrm{cm}^{3}}\right] \mathrm{eV},
$$




\begin{tabular}{|c|c|}
\hline NSI parameters & $2 \sigma$ Bounds \\
\hline$\varepsilon_{e \mu}$ & {$[-0.372,+0.301]$} \\
$\varepsilon_{e \tau}$ & {$[-1.657,+0.732]$} \\
$\varepsilon_{\mu \tau}$ & {$[-0.076,+0.058]$} \\
$\beta\left(\varepsilon_{\mu \mu}-\varepsilon_{e e}\right)$ & {$[-2.861,+0.144]$} \\
$\gamma\left(\varepsilon_{\tau \tau}-\varepsilon_{e e}\right)$ & {$[-2.892,+0.836]$} \\
\hline
\end{tabular}

Table 1. Bounds on the effective NC-NSI parameters from the neutrino oscillation experiments at $2 \sigma$ confidence level. Values of $\varepsilon_{\alpha \beta}^{f}$ in eq. (2.9) is taken from the global fit analysis [95].

where $Y_{e}=N_{e} /\left(N_{p}+N_{n}\right)$ is the relative electron number density of the medium and $\rho$ is the Earth matter density. For the Earth matter which is the focus of our paper, it is safe to assume neutral and isoscalar matter, i.e. $N_{n} \approx N_{p}=N_{e}$. Under these assumptions, the relative electron number density inside the Earth turns out to be $Y_{e} \approx 0.5$.

The $(1,1)$ element of the effective Hamiltonian $H_{f}$ (see eq. (2.5)) contains the term $\varepsilon_{e e} V_{C C}$ which gets simply added to the standard matter effect term. Since it can mimic the role of standard interaction, it is a wise choice to subtract a common physical phase $I\left(\equiv \varepsilon_{e e} V_{C C}\right)$ from the right-hand side (r.h.s.) of eq. (2.5). Then, the effective Hamiltonian takes the form

$$
H_{f}=\Delta_{31}\left[U\left(\begin{array}{lll}
0 & 0 & 0 \\
0 & \alpha & 0 \\
0 & 0 & 1
\end{array}\right) U^{\dagger}+\hat{A}\left(\begin{array}{ccc}
1 & \varepsilon_{e \mu} & \varepsilon_{e \tau} \\
\varepsilon_{e \mu}^{*} & \beta & \varepsilon_{\mu \tau} \\
\varepsilon_{e \tau}^{*} & \varepsilon_{\mu \tau}^{*} & \gamma
\end{array}\right)\right]
$$

where, $\Delta_{31} \equiv \Delta m_{31}^{2} / 2 E, \alpha \equiv \Delta m_{21}^{2} / \Delta m_{31}^{2}, \hat{A} \equiv 2 E V_{C C} / \Delta m_{31}^{2}$. We define the effective lepton-flavor-conserving diagonal NC-NSI parameters as $\beta \equiv \varepsilon_{\mu \mu}-\varepsilon_{e e}$ and $\gamma \equiv \varepsilon_{\tau \tau}-\varepsilon_{e e}$.

We now briefly discuss the present constraints on the effective NC-NSI parameters obtained from the global fit of neutrino oscillation data [95]. Using eq. (2.4), we can write,

$$
\begin{aligned}
\varepsilon_{\alpha \beta} & =\varepsilon_{\alpha \beta}^{p}+Y_{n} \varepsilon_{\alpha \beta}^{n} \\
& =\left(2+Y_{n}\right) \varepsilon_{\alpha \beta}^{u}+\left(1+2 Y_{n}\right) \varepsilon_{\alpha \beta}^{d},
\end{aligned}
$$

where, $Y_{n}$ is the average neutron/proton ratio inside the Earth. According to ref. [95], $Y_{n}=1.051$. Here, we have taken into account the fact that $N_{u}=2 N_{p}+N_{n}$ and $N_{d}=$ $N_{p}+2 N_{n}$, which in turn imply that $\varepsilon_{\alpha \beta}^{p}=2 \varepsilon_{\alpha \beta}^{u}+\varepsilon_{\alpha \beta}^{d}$ and $\varepsilon_{\alpha \beta}^{n}=\varepsilon_{\alpha \beta}^{u}+2 \varepsilon_{\alpha \beta}^{d}$. Note that the contribution from $\varepsilon_{\alpha \beta}^{e}$ is not considered in the global $3 \nu$ analysis in the presence of NC-NSI parameters [95]. Now, we use the bounds $(2 \sigma)$ on $\varepsilon_{\alpha \beta}^{u}$ and $\varepsilon_{\alpha \beta}^{d}$ from the global fit analysis [95] and list the subsequent $2 \sigma$ bounds on the effective NSI parameters $\varepsilon_{\alpha \beta}$ in table 1 .

\section{Diagonalization of the effective Hamiltonian in the presence of NSI}

Here, we derive the approximate analytical expressions for the fundamental oscillation parameters in matter considering all possible lepton-flavor-conserving and lepton-flavor- 
violating $\mathrm{NC}^{-\mathrm{NSI}^{3}}$ which are real i.e., all the phases associated with the non-diagonal elements of the NSI matrix are assumed to be zero.

In order to simplify the subsequent calculations, here, we perform our analysis in the CP-conserving scenario i.e., we take the standard Dirac CP phase $\delta_{\mathrm{CP}}$ to be zero. In appendix $\mathrm{B}$, we also perform the same considering the non-zero values of $\delta_{\mathrm{CP}}$. The elements of the effective Hamiltonian $H_{f}$ in eq. (2.8) are then given by,

$$
\begin{aligned}
& \left(H_{f}\right)_{11}=\Delta_{31}\left[\alpha s_{12}^{2} c_{13}^{2}+s_{13}^{2}+\hat{A}\right] \\
& \left(H_{f}\right)_{12}=\frac{\Delta_{31}}{2}\left[\sin 2 \theta_{13} s_{23}\left(1-\alpha s_{12}^{2}\right)+\alpha \sin 2 \theta_{12} c_{13} c_{23}+2 \varepsilon_{e \mu} \hat{A}\right] \\
& \left(H_{f}\right)_{13}=\frac{\Delta_{31}}{2}\left[\sin 2 \theta_{13} c_{23}\left(1-\alpha s_{12}^{2}\right)-\alpha \sin 2 \theta_{12} c_{13} s_{23}+2 \varepsilon_{e \tau} \hat{A}\right] \\
& \left(H_{f}\right)_{22}=\frac{\Delta_{31}}{2}\left[\alpha c_{12}^{2}+c_{13}^{2}+\alpha s_{12}^{2} s_{13}^{2}+\cos 2 \theta_{23}\left(\alpha c_{12}^{2}-\alpha s_{12}^{2} s_{13}^{2}-c_{13}^{2}\right)\right. \\
& \left.-\alpha \sin 2 \theta_{12} s_{13} \sin 2 \theta_{23}+2 \beta \hat{A}\right] \\
& \left(H_{f}\right)_{23}=\frac{\Delta_{31}}{2}\left[\sin 2 \theta_{23}\left(c_{13}^{2}-\alpha c_{12}^{2}+\alpha s_{12}^{2} s_{13}^{2}\right)-\alpha \sin 2 \theta_{12} s_{13} \cos 2 \theta_{23}+2 \varepsilon_{\mu \tau} \hat{A}\right] \\
& \left(H_{f}\right)_{33}=\frac{\Delta_{31}}{2}\left[\alpha c_{12}^{2}+c_{13}^{2}+\alpha s_{12}^{2} s_{13}^{2}+\cos 2 \theta_{23}\left(c_{13}^{2}-\alpha c_{12}^{2}+\alpha s_{12}^{2} s_{13}^{2}\right)\right. \\
& \left.+\alpha \sin 2 \theta_{12} s_{13} \sin 2 \theta_{23}+2 \gamma \hat{A}\right]
\end{aligned}
$$

In the above expressions, we use the abbreviations: $\cos \theta_{i j} \rightarrow c_{i j}, \sin \theta_{i j} \rightarrow s_{i j}$, and retain the terms of all orders in $\sin \theta_{13}$ and $\alpha$ which are quite essential in light of the large value of $\theta_{13}$. To find the effective mixing angles and mass-squared differences in the presence of Earth matter potential $\left(V_{C C}\right)$ and all possible NC-NSI parameters, we need to diagonalize the effective Hamiltonian $H_{f}$ in eq. (2.8). We approximately diagonalize $H_{f}$ by applying three successive rotations $R_{23}\left(\theta_{23}^{m}\right), R_{13}\left(\theta_{13}^{m}\right)$, and $R_{12}\left(\theta_{12}^{m}\right)$, where $R_{i j}\left(\theta_{i j}^{m}\right)$ is the rotation matrix for the $(i, j)$ block with the rotation angle $\theta_{i j}^{m}$. The product of these rotation matrices construct a $3 \times 3$ unitary matrix

$$
\tilde{U} \equiv R_{23}\left(\theta_{23}^{m}\right) R_{13}\left(\theta_{13}^{m}\right) R_{12}\left(\theta_{12}^{m}\right),
$$

such that it can almost diagonalize $H_{f}$

$$
\tilde{U}^{T} H_{f} \tilde{U} \simeq \operatorname{Diag}\left(m_{1, m}^{2} / 2 E, m_{2, m}^{2} / 2 E, m_{3, m}^{2} / 2 E\right)
$$

where, the off-diagonal terms after the final rotation are quite small $\left(\sim 10^{-8}\right)$ and can be safely neglected. ${ }^{4}$

\footnotetext{
${ }^{3}$ The authors in ref. [96] derived similar expressions in the context of a particular beyond the Standard Model (BSM) scenario where they considered the presence of long-range flavor-diagonal NSI appearing due to abelian $L_{e}-L_{\mu}$ symmetry. In the present work, we adopt a model independent approach and introduce all possible NSI parameters at-a-time in the framework. It allows us to study the evolution of mass-mixing parameters in a more generalized scheme considering all possible NSI parameters.

${ }^{4}$ After the final rotation, $(1,3)$ and $(2,3)$ elements of the rotated effective Hamiltonian remain non-zero. Simplifying the expressions of these non-zero elements, we find that these two elements are of the order $O\left(\Delta m_{31}^{2} \times \alpha^{2}\right)$ and $O\left(\Delta m_{31}^{2} \times \alpha s_{13}^{2}\right)$. Compared to these, the diagonal elements are approximately of the order $O\left(\Delta m_{31}^{2}\right)$ at relevant energies. So, it is safe to neglect these two off-diagonal elements and consider that the effective Hamiltonian is approximately diagonal.
} 
Below, we give the expressions for the mixing angles in matter that we derive by equating the small off-diagonal elements to zero after each rotation during the diagonalization process:

$$
\begin{aligned}
& \tan 2 \theta_{23}^{m} \simeq \frac{\left(c_{13}^{2}-\alpha c_{12}^{2}+\alpha s_{12}^{2} s_{13}^{2}\right) \sin 2 \theta_{23}-\alpha s_{13} \sin 2 \theta_{12} \cos 2 \theta_{23}+2 \varepsilon_{\mu \tau} \hat{A}}{\left(c_{13}^{2}-\alpha c_{12}^{2}+\alpha s_{12}^{2} s_{13}^{2}\right) \cos 2 \theta_{23}+\alpha s_{13} \sin 2 \theta_{12} \sin 2 \theta_{23}+(\gamma-\beta) \hat{A}} \\
& \tan 2 \theta_{13}^{m} \simeq \frac{\sin 2 \theta_{13}\left(1-\alpha s_{12}^{2}\right) \cos \Delta \theta_{23}-\alpha \sin 2 \theta_{12} c_{13} \sin \Delta \theta_{23}+2\left(\varepsilon_{e \mu} s_{23}^{m}+\varepsilon_{e \tau} c_{23}^{m}\right) \hat{A}}{\left(\lambda_{3}-\hat{A}-\alpha s_{12}^{2} c_{13}^{2}-s_{13}^{2}\right)}, \\
& \tan 2 \theta_{12}^{m} \simeq \frac{c_{13}^{m}\left[\alpha \sin 2 \theta_{12} c_{13} \cos \Delta \theta_{23}+\sin 2 \theta_{13}\left(1-\alpha s_{12}^{2}\right) \sin \Delta \theta_{23}+2\left(\varepsilon_{e \mu} c_{23}^{m}-\varepsilon_{e \tau} s_{23}^{m}\right) \hat{A}\right]}{\left(\lambda_{2}-\lambda_{1}\right)},
\end{aligned}
$$

where, $\Delta \theta_{23} \equiv \theta_{23}-\theta_{23}^{m}$ is the deviation of the modified mixing angle $\theta_{23}$ from its vacuum value. In the above equations, $\lambda_{1}, \lambda_{2}$, and $\lambda_{3}$ take the following forms:

$$
\begin{aligned}
\lambda_{3}=\frac{1}{2} & {\left[c_{13}^{2}+\alpha c_{12}^{2}+\alpha s_{12}^{2} s_{13}^{2}+(\beta+\gamma) \hat{A}\right.} \\
& \left.+\frac{(\gamma-\beta) \hat{A}+\alpha \sin 2 \theta_{12} s_{13} \sin 2 \theta_{23}+\left(c_{13}^{2}-\alpha c_{12}^{2}+\alpha s_{12}^{2} s_{13}^{2}\right) \cos 2 \theta_{23}}{\cos 2 \theta_{23}^{m}}\right], \\
\lambda_{2}=\frac{1}{2}\left[\alpha c_{12}^{2}+c_{13}^{2}+\alpha s_{12}^{2} s_{13}^{2}+(\beta+\gamma) \hat{A}\right. & \\
& \left.-\frac{(\gamma-\beta) \hat{A}+\alpha \sin 2 \theta_{12} s_{13} \sin 2 \theta_{23}+\left(c_{13}^{2}-\alpha c_{12}^{2}+\alpha s_{12}^{2} s_{13}^{2}\right) \cos 2 \theta_{23}}{\cos 2 \theta_{23}^{m}}\right], \\
\lambda_{1}=\frac{1}{2} & {\left[\lambda_{3}+\hat{A}+s_{13}^{2}+\alpha s_{12}^{2} c_{13}^{2}-\frac{\lambda_{3}-\hat{A}-s_{13}^{2}-\alpha s_{12}^{2} c_{13}^{2}}{\cos 2 \theta_{13}^{m}}\right] . }
\end{aligned}
$$

Note that throughout the entire paper, we consider the propagation of neutrinos inside the Earth and assume normal mass ordering ${ }^{5}$ (NMO). In case of antineutrino propagation, one has to reverse the sign of $V_{C C}$ in the above equations which in turn reverses the sign of $\hat{A}$. Similarly, to get the corresponding expressions for the inverted mass ordering (IMO), one has to flip the sign of $\alpha$ as well as the sign of $\hat{A}$ in eqs. (3.9) to (3.14). For the approximate analytical expressions of the modified mixing angles in non-zero $\delta_{\mathrm{CP}}$ scenario, see eqs. (B.1) to (B.3) in appendix B.

To check the validity of analytical expressions derived in this paper, we use the expressions for modified mixing angles as given in eqs. (3.9) to (3.14) and the expressions for modified mass-squared differences obtained using eqs. (5.1) to (5.3) (derived later in section 5) to calculate the oscillation probabilities analytically in the presence of all possible NC-NSI considering a four-layered ${ }^{6}$ model of Earth. Then, we compare our approximate

\footnotetext{
${ }^{5}$ There are two possible patterns of neutrino masses: a) $m_{3}>m_{2}>m_{1}$, called normal mass ordering (NMO) where $\Delta m_{31}^{2}>0$ and b) $m_{2}>m_{1}>m_{3}$, called inverted mass ordering (IMO) where $\Delta m_{31}^{2}<0$.

${ }^{6}$ In appendix C, we discuss in detail how we calculate various oscillation probabilities using our approximate analytical expressions for a given baseline which passes through different layers of Earth.
} 


\begin{tabular}{|c|c|c|c|c|c|}
\hline$\theta_{23}$ & $\theta_{13}$ & $\theta_{12}$ & $\delta_{\mathrm{CP}}$ & $\Delta m_{21}^{2}\left[\mathrm{eV}^{2}\right]$ & $\Delta m_{31}^{2}\left[\mathrm{eV}^{2}\right]$ \\
\hline $45^{\circ}$, & $8.61^{\circ}$ & $33.8^{\circ}$ & $0^{\circ}$ & $7.39 \times 10^{-5}$ & $2.52 \times 10^{-3}$ \\
\hline
\end{tabular}

Table 2. The values of the oscillation parameters used in our analysis. The values of the other parameters are consistent with the present best-fit values as obtained in various global fit studies [3-6]. We assume normal mass ordering (NMO) throughout the paper.

analytical oscillation probabilities with the exact numerical probabilities obtained from the GLoBES software [97, 98]. We discuss this in detail in appendix A. We observe that the analytical and numerical oscillation probabilities match quite well for wide range of energies and baselines even for NSI strengths as large as 0.3 .

\section{Evolution of mixing angles in the presence of NSI}

In the present section, we study in detail how the effective mixing angles in matter $\theta_{12}^{m}, \theta_{13}^{m}$, and $\theta_{23}^{m}$ (we derive their expressions in section 3) get modified as functions of energy and baseline in the presence of all possible NC-NSI. For this study, we consider the three-flavor vacuum oscillation parameters as given in table 2 . To show our results, we consider two benchmark values of the NSI parameters: 0.2 and -0.2 .

\subsection{Evolution of $\boldsymbol{\theta}_{23}^{m}$}

Approximate analytical expression describing the evolution of the effective mixing angle $\theta_{23}^{m}$ is given in eq. (3.9). We can further simplify this expression by neglecting the small terms which are proportional to $\alpha s_{13} \sim 10^{-3}$ in eq. (3.9), which enable us to extract the useful physics insights related to the evolution of $\theta_{23}^{m}$ in a more concise fashion. With this approximation, the expression showing the evolution of $\theta_{23}$ in matter in the presence of NSI takes the form

$$
\tan 2 \theta_{23}^{m} \simeq \frac{\left(c_{13}^{2}-\alpha c_{12}^{2}\right) \sin 2 \theta_{23}+2 \varepsilon_{\mu \tau} \hat{A}}{\left(c_{13}^{2}-\alpha c_{12}^{2}\right) \cos 2 \theta_{23}+(\gamma-\beta) \hat{A}},
$$

where, $\gamma-\beta=\varepsilon_{\tau \tau}-\varepsilon_{\mu \mu}$. Two important features emerge from this simplified expression.

- Only NSI parameters from the $(2,3)$ block $\left(\varepsilon_{\mu \tau}\right.$ and an effective NSI parameter $\left.\gamma-\beta \equiv \varepsilon_{\tau \tau}-\varepsilon_{\mu \mu}\right)$ of the NSI Hamiltonian contribute to the modification of $\theta_{23}^{m}$.

- In the limiting case of all NSI parameters equal to zero (which in this case removes the standard matter effect $\hat{A}$ also), one would get back the vacuum mixing angle (i.e., $\left.\theta_{23}^{m}=\theta_{23}\right)$ irrespective of energy, baseline, and the octant of $\theta_{23}$. In other words, it implies that $\theta_{23}^{m}$ does not run in the presence of standard matter effect. Note that in the exact expression of $\theta_{23}^{m}$ in eq. (3.9), due to the presence of the tiny terms proportional to $\alpha s_{13}, \theta_{23}^{m}$ slightly deviates from its vacuum value even in the presence of SI.

In figure 1, we show the evolution of $\theta_{23}^{m}$ (using eq. (3.9)) with energy in presence of NSI parameters $(\gamma-\beta), \varepsilon_{\mu \tau}$, taken one-at-a-time for a baseline corresponding to the DUNE 


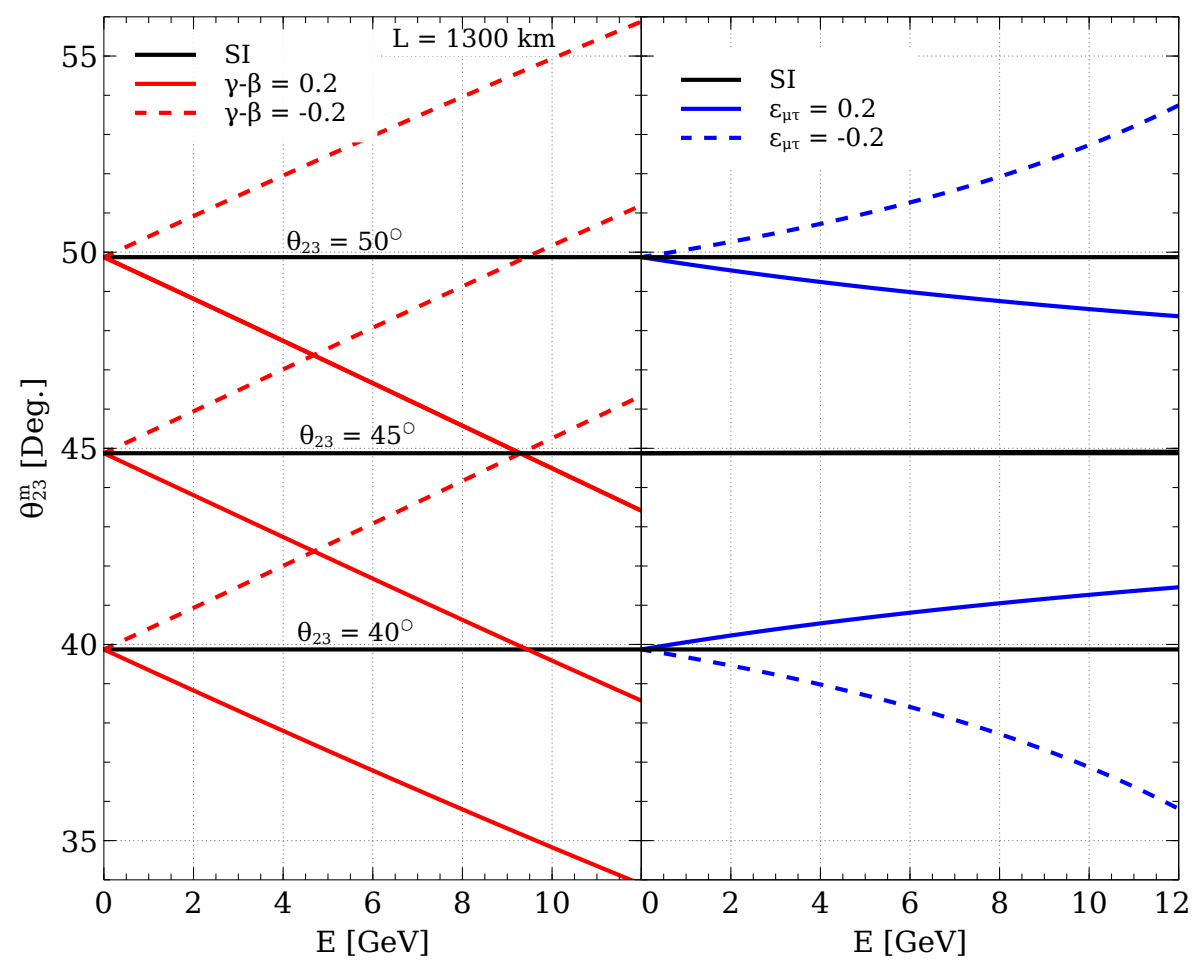

Figure 1. Evolution of $\theta_{23}^{m}$ in matter (given in eq. (3.9)) as a function of neutrino energy in the presence of SI and SI+NSI. Solid black curve in each panel represents the SI case while the other curves correspond to the SI+NSI cases with positive (solid lines) and negative (dashed lines) values of NSI parameters. In the left column, we show the modification in the presence of NSI parameter $(\gamma-\beta)$, while the right column depicts the effect of $\varepsilon_{\mu \tau}$. We consider $L=1300 \mathrm{~km}$ and assume NMO. We present results for three different values of $\theta_{23}$ in vacuum: $40^{\circ}$ (lower octant), $45^{\circ}$ (maximal value), $50^{\circ}$ (upper octant). The values of the other oscillation parameters in vacuum are taken from table 2.

experiment i.e. $1300 \mathrm{~km}$. The left column shows the effect of NSI parameter $(\gamma-\beta)$ while the right column corresponds to the effect of $\varepsilon_{\mu \tau}$. The black curves in each column depict the SI case for three possible values of $\theta_{23}$ in vacuum, namely higher octant $\left(\theta_{23}=50^{\circ}\right)$, maximal mixing $\left(\theta_{23}=45^{\circ}\right)$, and lower octant $\left(\theta_{23}=40^{\circ}\right)$. As discussed above, value of $\theta_{23}^{m}$ in SI case remains almost equal to the value of $\theta_{23}$ in vacuum. Only very small deviations from the vacuum value of $\theta_{23}$ can be observed due to the presence of terms proportional to $\alpha s_{13}$ in eq. (3.9), which are neglected in eq. (4.1). The solid (dashed) red curves in the left column of figure 1 illustrate the presence of $(\gamma-\beta)$ with a benchmark value of 0.2 $(-0.2)$. We observe that for all the three values of $\theta_{23}$ mentioned above, $\theta_{23}^{m}$ monotonically decreases (increases) with energy when $(\gamma-\beta)$ is present with a positive (negative) value. In the right column, the solid (dashed) blue curves depict the case when only $\varepsilon_{\mu \tau}$ is present with a benchmark value of $0.2(-0.2)$. Interestingly in lower (higher) octant, $\theta_{23}^{m}$ increases (decreases) for a positive value of $\varepsilon_{\mu \tau}$. For maximal mixing, the change in $\theta_{23}^{m}$ with energy is negligible in the presence of $\varepsilon_{\mu \tau}$ and remains almost equal to its vacuum value of $45^{\circ}$ (since the denominator of eq. (4.1) vanishes). The dependence of $\theta_{23}^{m}$ modification on the choice of octant of $\theta_{23}$ in vacuum can be understood from the fact that $\cos 2 \theta_{23}$ in the denominator of the r.h.s. of eq. (4.1) changes sign when $\theta_{23}$ lies in different octants. 


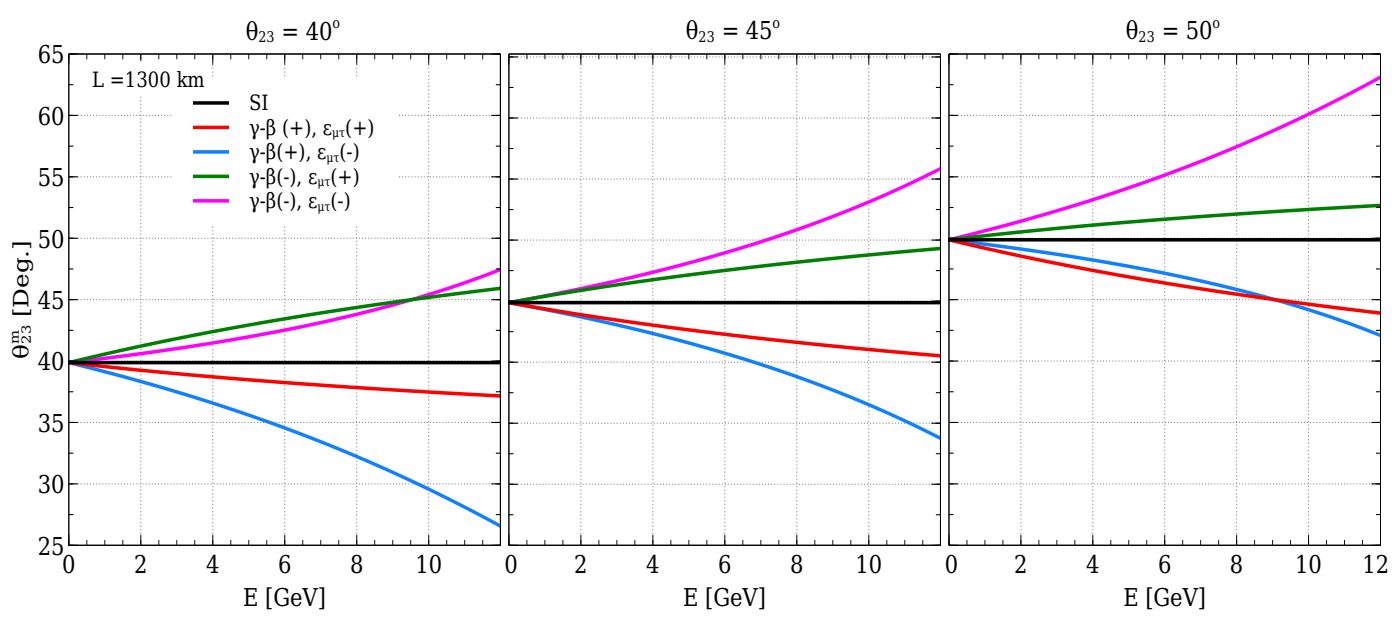

Figure 2. Evolution of $\theta_{23}^{m}$ (given in eq. (3.9)) with neutrino energy in matter with SI and NSI considering both $(\gamma-\beta)$ and $\varepsilon_{\mu \tau}$ non-zero at-a-time. Black curve in each column represents the SI case while the other curves show the cases with four possible combinations of the sign of $(\gamma-\beta)$ and $\varepsilon_{\mu \tau}$ with magnitude 0.2 . The left, middle, and right column correspond to the evolution considering three values of $\theta_{23}$ in vacuum, $40^{\circ}, 45^{\circ}$, and $50^{\circ}$, respectively. We consider $L=1300 \mathrm{~km}$ and assume NMO. Values of the oscillation parameters in vacuum used in this plot are taken from table 2.

Figure 2 shows the evolution of $\theta_{23}^{m}$ when both the NSI parameters $\varepsilon_{\mu \tau}$ and $(\gamma-\beta)$ are non-zero. The four colored curves in each panel illustrate the effect of the four possible sign combinations of $(\gamma-\beta)$ and $\varepsilon_{\mu \tau}$ while the black curve shows the SI (with standard matter effect and no NSI) case, as shown in the legend. As before, three scenarios of the vacuum mixing angle $\theta_{23}$ are considered: higher octant $\left(\theta_{23}=50^{\circ}\right)$, maximal mixing $\left(\theta_{23}=45^{\circ}\right)$, and lower octant $\left(\theta_{23}=40^{\circ}\right)$. We note from figure 2 that in the presence of $(\gamma-\beta)$ with a negative (positive) sign, $\theta_{23}^{m}$ monotonically increases (decreases) with energy irrespective of the sign of $\varepsilon_{\mu \tau}$ and the octant of $\theta_{23}$. We also observe that for lower (higher) octant, the decrease (increase) is the steepest when $(\gamma-\beta)$ is positive (negative) with negative value of $\varepsilon_{\mu \tau}$. For maximal mixing, the modification of $\theta_{23}^{m}$ appears symmetric around the SI case since the term with $\cos 2 \theta_{23}$ in the denominator of eq. (3.9) vanishes.

To show a correlation between NSI strength and the value of $\theta_{23}$ in vacuum, we have shown in figure 3 , the evolution of $\theta_{23}^{m}$ in the plane of $\left[\theta_{23}-\varepsilon_{\mu \tau}\right]$ (top panels) and $\left[\theta_{23}-(\gamma-\beta)\right]$ (bottom panels). We demonstrate the effect of baseline and energy by choosing three different baselines (energies) as $1300 \mathrm{~km}(2.6 \mathrm{GeV}), 5000 \mathrm{~km}(5 \mathrm{GeV})$, and $8000 \mathrm{~km}(8 \mathrm{GeV})$ in the three columns, respectively. For a given baseline, we consider energy closed to the first oscillation maximum for $\nu_{\mu} \rightarrow \nu_{e}$ appearance probability. For each of the baseline, we consider a line-averaged constant passes through the crust and mantle of the Earth. ${ }^{7}$ Earth matter density obtained from the PREM profile [99] of the Earth. For

\footnotetext{
${ }^{7}$ The baselines $1300 \mathrm{~km}\left(\rho_{\text {avg }}=2.88 \mathrm{~g} / \mathrm{cm}^{3}\right)$ and $5000 \mathrm{~km}\left(\rho_{\text {avg }}=3.589 \mathrm{~g} / \mathrm{cm}^{3}\right)$ pass through only crust and the baseline $8000 \mathrm{~km}\left(\rho_{\text {avg }}=4.351 \mathrm{~g} / \mathrm{cm}^{3}\right)$. Since the density of the Earth does not vary much inside the crust and mantle, we can consider line-averaged constant Earth matter densities for these baselines. Note that while calculating oscillation probabilities using our approximate analytical expressions for a given baseline, we always use four-layered model of the Earth as discussed in appendix C.
} 


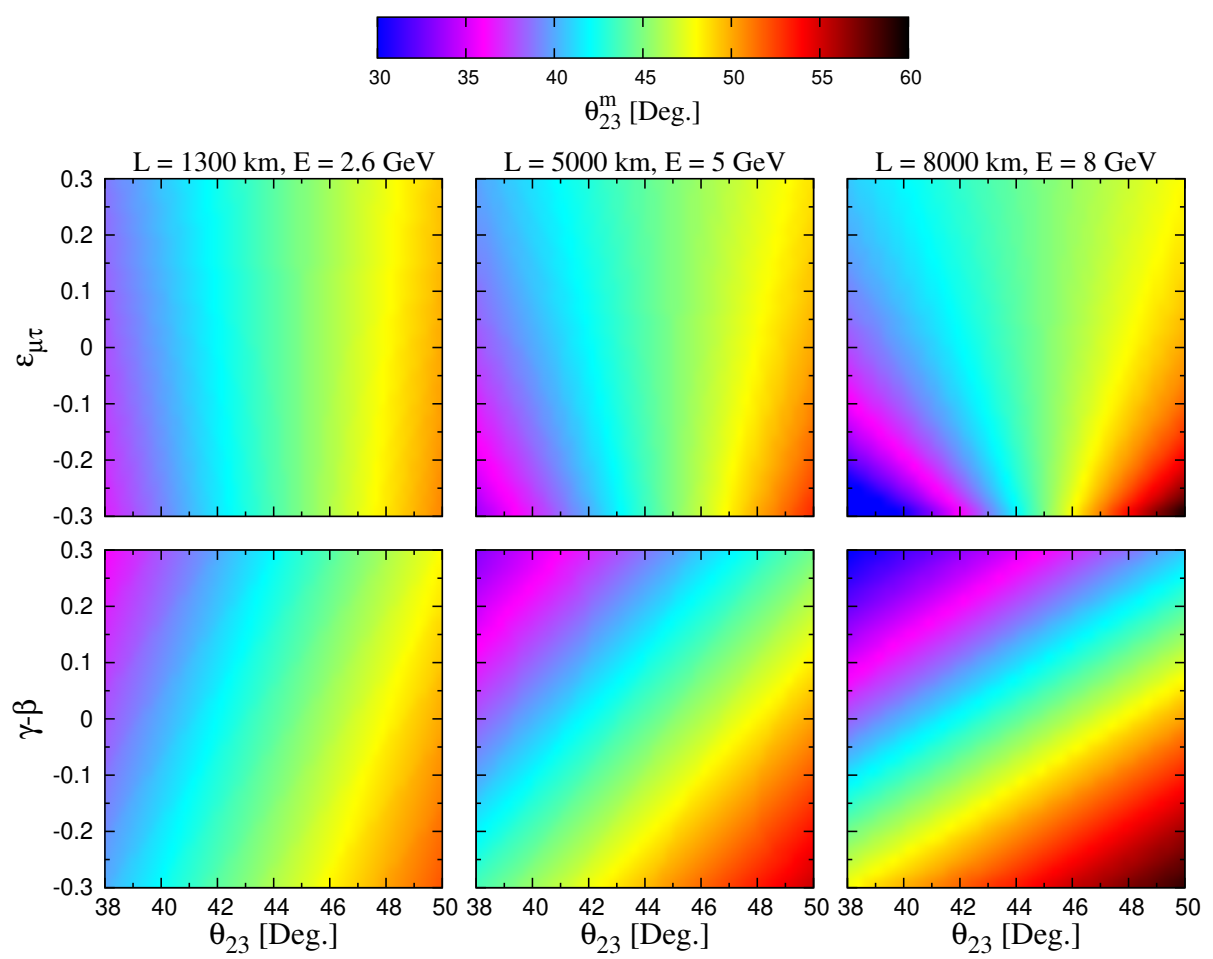

Figure 3. The evolution of $\theta_{23}^{m}$ are shown in the plane of $\left[\theta_{23}-\varepsilon_{\mu \tau}\right]$ (top row) and $\left[\theta_{23}-(\gamma-\beta)\right]$ (bottom row). The left, middle, and right columns correspond to three sets of baseline and neutrino energy, namely $(1300 \mathrm{~km}, 2.6 \mathrm{GeV}),(5000 \mathrm{~km}, 5 \mathrm{GeV})$, and $(8000 \mathrm{~km}, 8 \mathrm{GeV})$, respectively. Values of the oscillation parameters in vacuum used in this plot are taken from table 2 and we assume NMO.

the baseline of $1300 \mathrm{~km}$, we see that $\theta_{23}^{m}$ decreases (increases) from the vacuum value $\left(\theta_{23}\right)$ for a positive (negative) $\varepsilon_{\mu \tau}$ at higher octant. However, an opposite trend can be observed at lower octant. For maximal mixing, $\theta_{23}^{m}$ does not change in presence of $\varepsilon_{\mu \tau}$ only. These features are more pronounced for higher baselines since the NSI effect (proportional to matter density) gets enhanced. In the bottom row, in the presence of positive (negative) value of $(\gamma-\beta), \theta_{23}^{m}$ decreases (increases) from the vacuum value, irrespective of the octant or maximal mixing. Larger baselines manifest it more clearly as evident from the steeper slant of the boundaries between different colors.

As mentioned earlier, we have assumed normal mass ordering (NMO) for our analysis. In case of inverted mass ordering (IMO) with neutrino ( $\nu$, IMO), the effect of each NSI parameters in $\theta_{23}^{m}$ evolution is reversed (i.e., if $\theta_{23}^{m}$ increases with energy in presence of a particular NSI parameter with normal ordering of mass, in case of inverted mass ordering $\theta_{23}^{m}$ will decrease with energy). This happens since the term $\hat{A}$ associated with each NSI parameter changes its sign in case of IMO. Also, in case of antineutrino propagation with inverted mass ordering $\left(\bar{\nu}\right.$, IMO), the change in $\theta_{23}^{m}$ is almost the same as that of neutrino propagation with NMO $(\nu, \mathrm{NMO})$. This is because of the fact that in both cases, sign of $\hat{A}$ is the same. 


\subsection{Evolution of $\theta_{13}^{m}$}

Eq. (3.10) shows the evolution of $\theta_{13}^{m}$ in matter in the presence of NC-NSI parameters. We note that all five NSI parameters as well as the standard matter effect term ${ }^{8}(\hat{A})$ have impact on the evolution of $\theta_{13}^{m}$. It is observed that the value of $\theta_{23}$ in vacuum (when it lies in the range of $40^{\circ}$ to $50^{\circ}$ ) has a very small effect on the modification of $\theta_{13}^{m}$. So, we simplify the expression for our understanding by assuming that the mixing angle $\theta_{23}$ in vacuum is maximal i.e., $45^{\circ}$. The relevant expression for the $\theta_{13}^{m}$ thus becomes,

$$
\tan 2 \theta_{13}^{m} \simeq \frac{\sin 2 \theta_{13}\left(1-\alpha s_{12}^{2}\right)\left(s_{23}^{m}+c_{23}^{m}\right)-\alpha \sin 2 \theta_{12} c_{13}\left(c_{23}^{m}-s_{23}^{m}\right)+2 \sqrt{2}\left(\varepsilon_{e \mu} s_{23}^{m}+\varepsilon_{e \tau} c_{23}^{m}\right) \hat{A}}{\sqrt{2}\left(\lambda_{3}-\hat{A}-\alpha s_{12}^{2} c_{13}^{2}-s_{13}^{2}\right)}
$$

where,

$$
\lambda_{3}=\frac{1}{2}\left[c_{13}^{2}+\alpha c_{12}^{2}+\alpha s_{12}^{2} s_{13}^{2}+(\beta+\gamma) \hat{A}+\frac{(\gamma-\beta) \hat{A}+\alpha \sin 2 \theta_{12} s_{13}}{\cos 2 \theta_{23}^{m}}\right] .
$$

In figure 4, we show the modification of $\theta_{13}^{m}$ with energy (by using eqs. (4.2) and (4.3)) in presence of NSI for a baseline of $1300 \mathrm{~km}$ and $\theta_{23}=45^{\circ}$. The SI case is depicted by the black curve in each panel and the other colored curves indicate the presence of NSI parameters in matter with a benchmark strength of 0.2 and -0.2 . In the top row, we have shown the variation of $\theta_{13}^{m}$ when NSI are positive. The top left panel illustrates the presence of NSI parameters in $(2,3)$ block while the right shows the effect of $\varepsilon_{e \mu}$ and $\varepsilon_{e \tau}$. We note that unlike the case of $\theta_{23}^{m}, \theta_{13}^{m}$ runs even in presence of only SI - its value rapidly rising with energy from the vacuum value of $\theta_{13}=8.5^{\circ}$. This can be understood from the fact that with an increase in energy, the term $\left(\lambda_{3}-\hat{A}\right)$ in the denominator of the r.h.s. in eq. (4.2) becomes smaller. The NSI parameters from $(2,3)$ block suppress the rapid rise to some extent due to the modification in the value of $\lambda_{3}$ (see eq. (4.3)). Moreover, presence of $\beta$ or $\gamma$ only with the same strength, makes $\theta_{13}^{m}$ run in identical manner. ${ }^{9}$ On the other hand, $\varepsilon_{e \mu}$ and/or $\varepsilon_{e \tau}$ increases the magnitude of $\theta_{13}^{m}$ due to the additional contribution in the numerator of the r.h.s. in eq. (4.2). For the case of maximal mixing of $\theta_{23}$, the impact of $\varepsilon_{e \mu}$ is identical to that of $\varepsilon_{e \tau}$ since $\theta_{23}^{m} \simeq \theta_{23}=45^{\circ}$. At lower energy, the gap between the curves showing the evolution in the presence of $\varepsilon_{e \mu} / \varepsilon_{e \tau}$ and the SI case increase with energy. However, as the value of $\theta_{13}^{m}$ approaches $45^{\circ}$, the gap becomes narrower and at $\theta_{13}^{m}=45^{\circ}$, these three curves intersect. It happens because, around value of $\theta_{13}^{m} \approx 45^{\circ}$ denominator of r.h.s. in eq. (4.2) becomes so small that the effect from the numerator which have $\varepsilon_{e \mu} / \varepsilon_{e \tau}$ is insignificant. In the bottom row, we have shown $\theta_{13}^{m}$ evolution for the negative values of the NSI parameters. It is clear from the bottom left panel that variation of $\theta_{13}^{m}$ is enhanced when NSI from $(2,3)$ block is present with negative strength. This happens since the presence of these negative NSI parameters decrease the value of $\lambda_{3}$, thereby decreasing the overall value of the denominator of r.h.s. in eq. (4.2). In the bottom right panel, some non-trivial effects are observed. We see that negative $\varepsilon_{e \mu}$ or $\varepsilon_{e \tau}$ highly suppresses the modification of

\footnotetext{
${ }^{8}$ Note that the standard matter effect term $\hat{A}$ alone does not affect the variation of $\theta_{23}^{m}$ (see eq. (4.1)).

${ }^{9}$ From the discussion of subsection 4.1 , we know that $\cos 2 \theta_{23}^{m}$ is consistently positive (negative with the same magnitude) in presence of a positive $\beta(\gamma)$ throughout $E>0$. Thus $\lambda_{3}$ in eq. (4.3) remains the same in presence of $\beta$ or $\gamma$ with the same strength.
} 


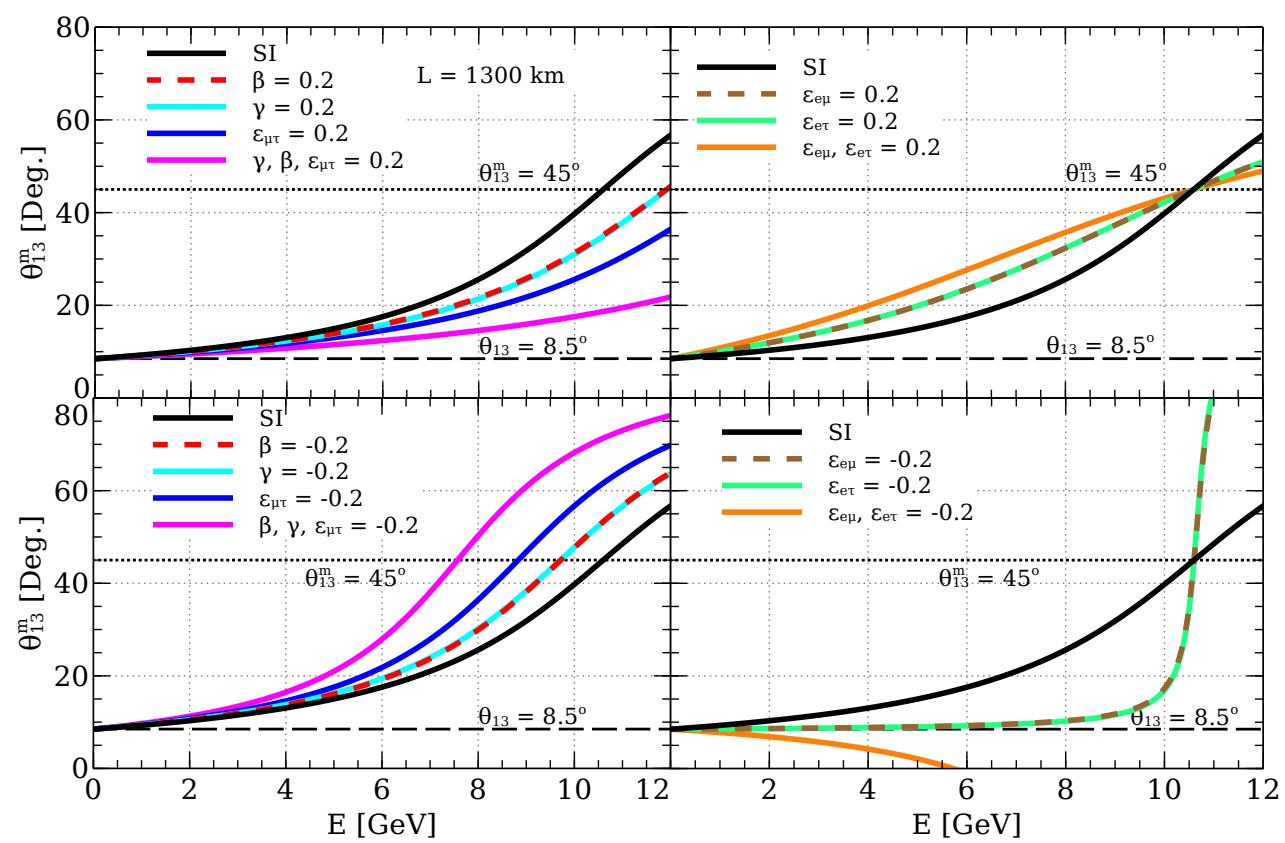

Figure 4. Evolution of $\theta_{13}^{m}$ (given in eq. (4.2)) with energy in presence of SI and NSI in matter. The solid black curve in each panel shows the SI case while the other curves correspond to the variation in the presence of SI+NSI. In the top (bottom) row, the NSI have been considered with a benchmark value of $0.2(-0.2)$. The left column depicts the presence of NSI parameters in $(2,3)$ block while the right column shows the effect of $\varepsilon_{e \mu}$ and $\varepsilon_{e \tau}$. We have used $L=1300 \mathrm{~km}$ and assumed NMO in the plot. Values of oscillation parameters in vacuum are given in table 2 with $\theta_{23}=45^{\circ}$.

$\theta_{13}^{m}$ such that at lower energy $(E \lesssim 6 \mathrm{GeV}$ ), it is almost constant when only one of them is present. It can be explained by the fact that both numerator and denominator of r.h.s. in eq. (4.2) decreases with energy when $\varepsilon_{e \mu}$ and/or $\varepsilon_{e \tau}$ are negative, such that the overall value of $\theta_{13}^{m}$ remains almost constant at that energy range. However, at higher energy $(\sim$ $10 \mathrm{GeV}$ ) value of the denominator is so small that the overall effect led to the rapid increase in the magnitude of $\theta_{13}^{m}$ with energy. As we can see from eq. (4.2), in presence of both $\varepsilon_{e \mu}$ and $\varepsilon_{e \tau}$ with a negative sign, the numerator decreases faster with energy compared to the previous case due to the additive effect of two NSI parameters. Consequently, value of $\theta_{13}^{m}$ decreases with energy from its vacuum value, and becomes negative (at $E \gtrsim 6 \mathrm{GeV}$ ) when the numerator becomes negative.

In the case of IMO, the behavior of $\theta_{13}^{m}$ in SI as well as in SI+NSI case is significantly different from the NMO case for neutrino. It can be understood from the $\left(\lambda_{3}-\hat{A}\right)$ term in the denominator of eq. (4.2). Since $\hat{A}$ changes its sign, the denominator increases with energy, consequently the value of the $\theta_{13}^{m}$ decreases from its vacuum value. However, in case of antineutrino $(\bar{\nu})$ propagation and inverted mass ordering $(\bar{\nu}$, IMO), since $\hat{A}$ does not change its sign, variation of $\theta_{13}^{m}$ is almost similar to neutrino $(\nu, \mathrm{NMO})$ case.

\subsection{Evolution of $\theta_{12}^{m}$}

Similar to the case of $\theta_{13}^{m}$, value of $\theta_{23}$ in vacuum (when it is between $40^{\circ}$ and $50^{\circ}$ ) also has very small impact in the modification of $\theta_{12}^{m}$. With the assumption of maximal mixing of 
$\theta_{23}$, the relevant expression for $\theta_{12}^{m}$ in eq. (3.11) takes the form

$$
\tan 2 \theta_{12}^{m} \simeq \frac{c_{13}^{m}\left[\alpha \sin 2 \theta_{12} c_{13}\left(c_{23}^{m}+s_{23}^{m}\right)+\sin 2 \theta_{13}\left(1-\alpha s_{12}^{2}\right)\left(c_{23}^{m}-s_{23}^{m}\right)+2 \sqrt{2}\left(\varepsilon_{e \mu} c_{23}^{m}-\varepsilon_{e \tau} s_{23}^{m}\right) \hat{A}\right]}{\sqrt{2}\left(\lambda_{2}-\lambda_{1}\right)},
$$

where,

$$
\begin{aligned}
& \lambda_{2}=\frac{1}{2}\left[\alpha c_{12}^{2}+c_{13}^{2}+\alpha s_{12}^{2} s_{13}^{2}+(\beta+\gamma) \hat{A}-\frac{(\gamma-\beta) \hat{A}+\alpha \sin 2 \theta_{12} s_{13}}{\cos 2 \theta_{23}^{m}}\right], \\
& \lambda_{1}=\frac{1}{2}\left[\lambda_{3}+\hat{A}+s_{13}^{2}+\alpha s_{12}^{2} c_{13}^{2}-\frac{\lambda_{3}-\hat{A}-s_{13}^{2}-\alpha s_{12}^{2} c_{13}^{2}}{\cos 2 \theta_{13}^{m}}\right] .
\end{aligned}
$$

In figure 5 , the evolution of $\theta_{12}^{m}$ with energy is shown both for SI (black curve) and for SI+NSI parameters (other curves) for a baseline of $1300 \mathrm{~km}$ and $\theta_{23}=45^{\circ}$. The left column shows the effect of the NSI parameters in $(2,3)$ block, while the right column depicts the case of $\varepsilon_{e \mu}$ and $\varepsilon_{e \tau}$ with a strength of 0.2 or -0.2 . For SI, at small energies $(E \lesssim 1.5-2 \mathrm{GeV}), \lambda_{1}$ being close to $\lambda_{2}, \theta_{12}^{m}$ shows a very steep increase and then quickly saturates and approaches to $90^{\circ}$ approximately. ${ }^{10}$ Saturation occurs due to the following two reasons.

1. With increase in energy, $\lambda_{1}$ moves away from $\lambda_{2}$, resulting in a large denominator in the r.h.s. of eq. (4.4).

2. $\theta_{13}^{m}$ rises with energy (see figure 4 and the relevant discussions in subsection 4.2) and so the overall factor $c_{13}^{m}$ in eq. (4.4) decreases.

In the presence of NSI parameters in $(2,3)$ block, $\lambda_{1}, \lambda_{2}$ and $c_{13}^{m}$ undergo mild change, retaining almost the same features as that of SI. The presence of $\varepsilon_{e \mu}\left(\varepsilon_{e \tau}\right)$ however, adds up to the numerator of eq. (4.4) ${ }^{11}$ and the value of $\theta_{12}^{m}$ at which it saturates, shifts down (up). When both $\varepsilon_{e \mu}$ and $\varepsilon_{e \tau}$ are present, they cancel their effect due to the relative sign between them and the evolution of $\theta_{12}^{m}$ almost coincides with SI scenario. In the bottom row, we have shown the modification of $\theta_{12}^{m}$ for the NSI with negative strength. Since $\theta_{12}^{m}$ very mildly depend on NSI parameters from the $(2,3)$ sector (bottom left panel), the sign of these NSI parameters do not have any significant effect. In the bottom right panel, we see that role of $\varepsilon_{e \mu}$ and $\varepsilon_{e \tau}$ is reversed when the sign of the NSI parameter is changed. Interestingly, at energies around $10 \mathrm{GeV}$, sudden decrease (increase) of $\theta_{12}^{m}$ can be observed in the presence of NSI parameter $\varepsilon_{e \mu}\left(\varepsilon_{e \tau}\right)$ with negative strength. It happens due to the presence of the term $\cos \theta_{13}^{m}$ in the numerator of the r.h.s. of eq. (4.4), which reduces rapidly to a very small value around that energy (see figure 4 and related discussion in subsection 4.2).

Unlike $\theta_{13}^{m}$, the $\theta_{12}^{m}$ shows similar behavior in SI as well as in SI+NSI cases for neutrino propagation with IMO $(\nu$, IMO). Also, it shows completely different behavior in case of

\footnotetext{
${ }^{10}$ Note that here we assume modified mass eigenstates $m_{2, m}$ is always greater than the $m_{1, m}$ to ensure that the evolution of masses are continuous as a function of the matter potential and $\theta_{12}^{m}$ can also approach $90^{\circ}$ in matter.

${ }^{11}$ With increase in energy, $\lambda_{2}-\lambda_{1}$ in the denominator of eq. (4.4) becomes negative. So a positive (negative) contribution to the numerator by $\varepsilon_{e \mu}\left(\varepsilon_{e \tau}\right)$ decreases (increases) the magnitude of $\theta_{12}^{m}$.
} 


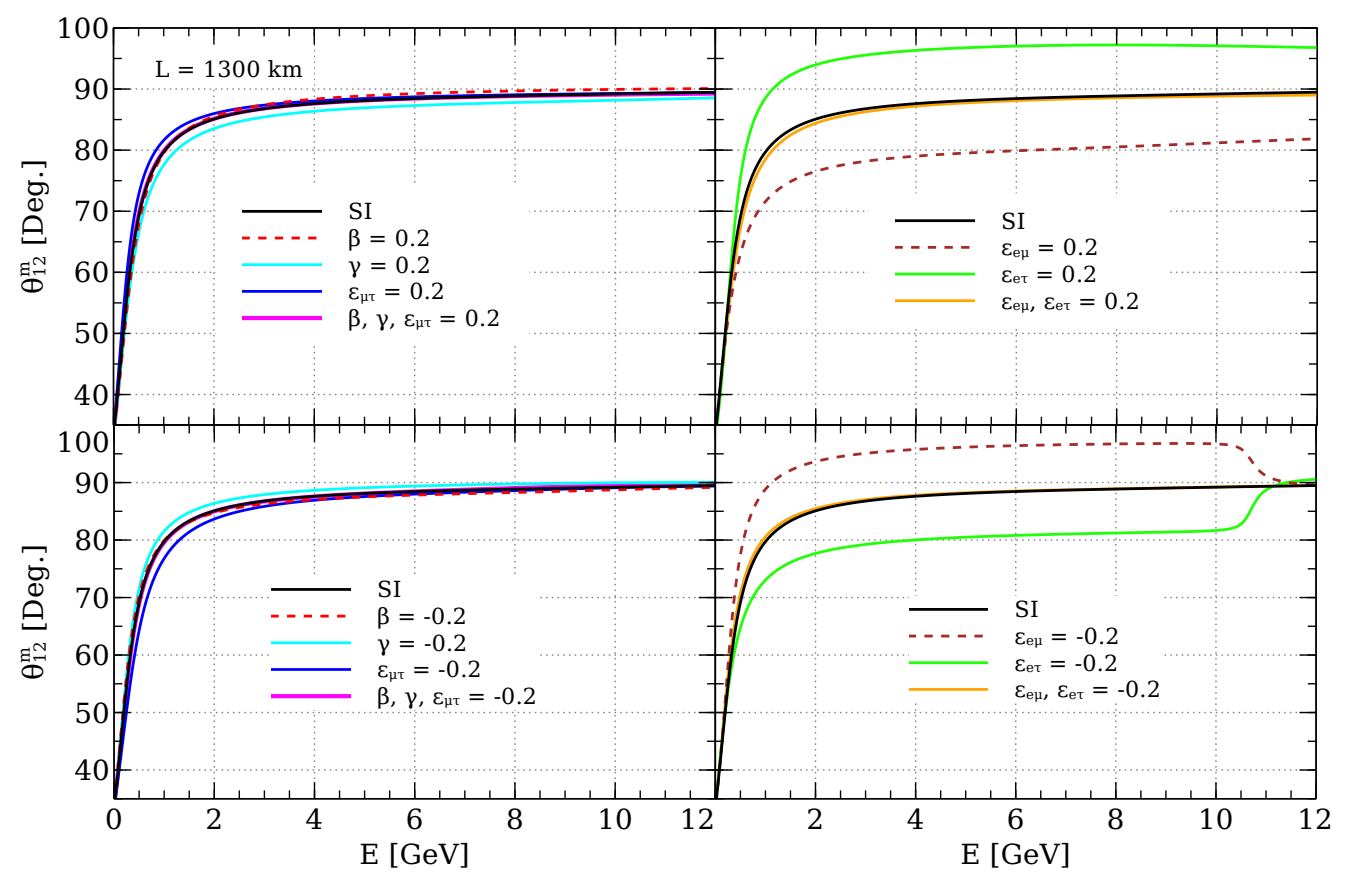

Figure 5. Evolution of $\theta_{12}^{m}$ (given in eq. (4.4)) with energy in presence of SI and NSI in matter. The solid black curve in each panel shows the SI case while the other curves correspond to the variation in the presence of SI+NSI. In the top (bottom) row, the positive (negative) values of the NSI are considered with a benchmark value of $0.2(-0.2)$. The left column depicts the presence of NSI parameters in $(2,3)$ block while the right shows the effect of $\varepsilon_{e \mu}$ and $\varepsilon_{e \tau}$. We consider $L=1300 \mathrm{~km}$ and assume NMO to prepare this plot. Values of oscillation parameters in vacuum used in this plot are taken from table 2 with $\theta_{23}=45^{\circ}$.

antineutrino propagation with inverted mass ordering $(\bar{\nu}$, IMO). It can be understood from the fact that in case of IMO, sign of first and third terms in the numerator of eq. (4.4) gets flipped, and in the denominator, the sign of $\lambda_{1}$ gets changed. Since the effect from other remaining terms are very small, both numerator and denominator change their sign, and as a result, $\theta_{12}^{m}$ remains the same as in the case of $(\nu, \mathrm{NMO})$. In case of $(\bar{\nu}$, IMO), only first term in the numerator changes its sign, $\lambda_{1}$ in the denominator remains the same as in case of $(\nu, \mathrm{NMO})$. As a result, we see a completely different behavior of $\theta_{12}^{m}$.

\section{Evolution of mass-squared differences in the presence of NSI}

After the diagonalization of the effective propagation Hamiltonian $H_{f}$ in section 2, we obtain the expressions for the eigenvalues $m_{i, m}^{2} / 2 E(i=1,2,3)$ :

$$
\begin{aligned}
\frac{m_{3, m}^{2}}{2 E} & \simeq \frac{\Delta_{31}}{2}\left[\lambda_{3}+\hat{A}+s_{13}^{2}+\alpha s_{12}^{2} c_{13}^{2}+\frac{\lambda_{3}-\hat{A}-s_{13}^{2}-\alpha s_{12}^{2} c_{13}^{2}}{\cos 2 \theta_{13}^{m}}\right], \\
\frac{m_{2, m}^{2}}{2 E} & \simeq \frac{\Delta_{31}}{2}\left[\lambda_{1}+\lambda_{2}-\frac{\lambda_{1}-\lambda_{2}}{\cos 2 \theta_{12}^{m}}\right], \\
\frac{m_{1, m}^{2}}{2 E} & \simeq \frac{\Delta_{31}}{2}\left[\lambda_{1}+\lambda_{2}+\frac{\lambda_{1}-\lambda_{2}}{\cos 2 \theta_{12}^{m}}\right],
\end{aligned}
$$




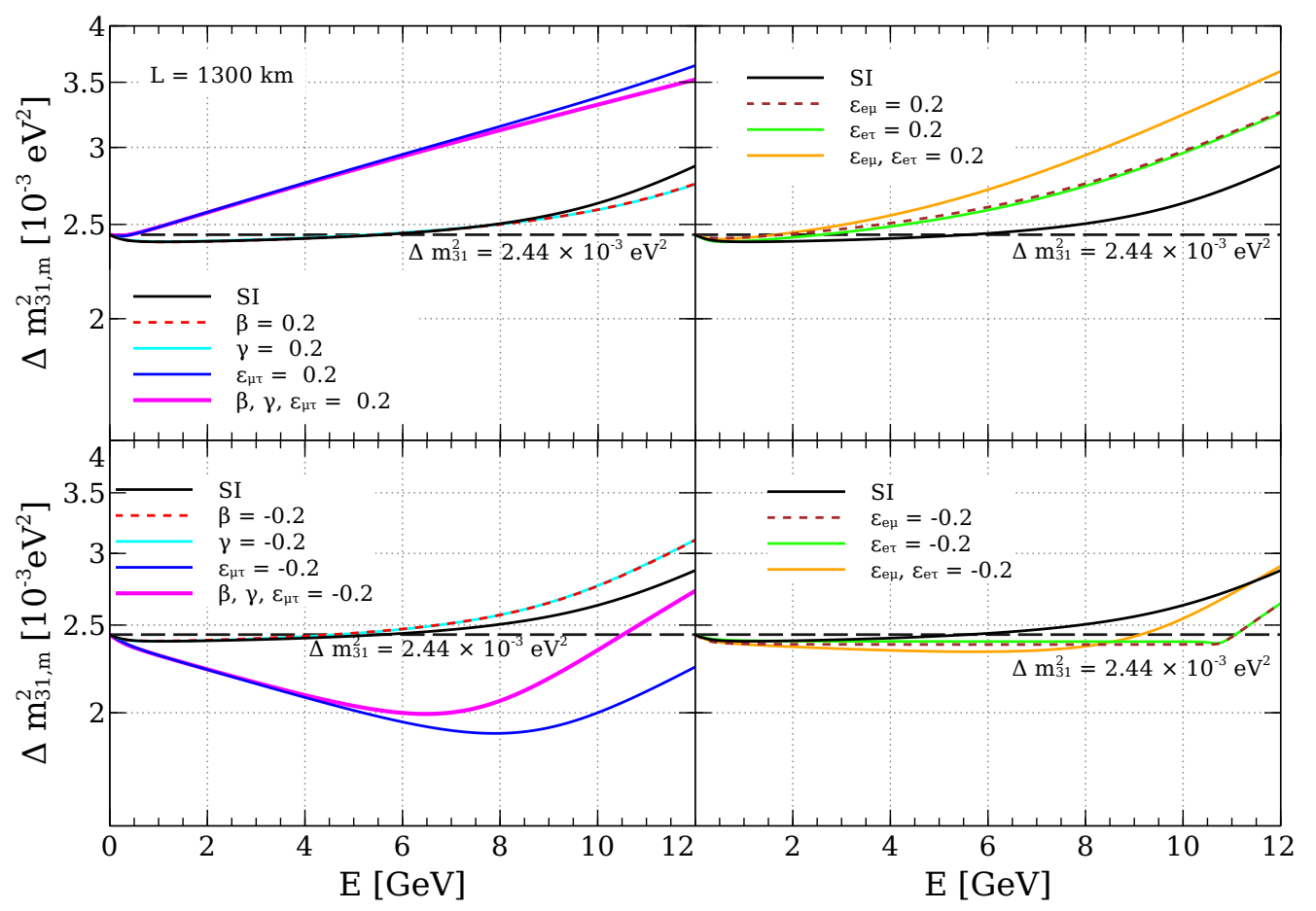

Figure 6. Variation of $\Delta m_{31, m}^{2}\left(\equiv m_{3, m}^{2}-m_{1, m}^{2}\right)$ as obtained from eqs. (5.1)-(5.3) is shown with energy in SI case and SI+NSI cases. Top (bottom) row corresponds to the positive (negative) NSI with strength 0.2. The solid black curve in each panel shows the SI case while the other curves show the modification in presence of NSI. The left column depicts the presence of various NSI parameters in $(2,3)$ block while the right shows the effect of $\varepsilon_{e \mu}$ and $\varepsilon_{e \tau}$. We consider $L=1300 \mathrm{~km}$ and the values of the oscillation parameters used in this plot are taken from table 2 . We assume $\theta_{23}=45^{\circ}$ and NMO.

where, we assume $\theta_{23}=45^{\circ}$ and we are already familiar with the expressions of $\theta_{i j}^{m}$ and $\lambda_{i}$. Using the above equations, we can obtain the approximate analytical expressions for the modified mass-squared differences $\Delta m_{31, m}^{2} \equiv m_{3, m}^{2}-m_{1, m}^{2}$ and $\Delta m_{21, m}^{2} \equiv m_{2, m}^{2}-m_{1, m}^{2}$. The behavior of $\Delta m_{31, m}^{2}\left(\Delta m_{21, m}^{2}\right)$ is mainly governed by $m_{3, m}^{2}\left(m_{2, m}^{2}\right)$. This is due to the fact that in the approximation $\theta_{12}^{m}$ saturating to $90^{\circ}$ (see subsection 4.3 ), $m_{2, m}^{2} \approx \lambda_{1} \Delta_{31}$ and $m_{1, m}^{2} \approx \lambda_{2} \Delta_{31}$. Therefore, $\lambda_{2}$ being very small, $m_{1, m}^{2}$ is insignificant. As we have already mentioned, we assume that $m_{2, m}$ is always greater than $m_{1, m}$ in matter such that the evolution of masses are continuous as we vary the matter potential.

In figure 6 , we show the evolution of $\Delta m_{31, m}^{2}$ both for SI (black curve) and SI+NSI (other colored curves) scenarios. The top (bottom) row corresponds to the variation in the presence of positive (negative) NSI with strength 0.2. The left column depicts the presence of various NSI parameters in $(2,3)$ block while the right shows the effect of $\varepsilon_{e \mu}$ and $\varepsilon_{e \tau}$. A baseline of $1300 \mathrm{~km}$ and a maximal mixing for $\theta_{23}$ is considered. For the SI case, $\Delta m_{31, m}^{2}$ first increases very slowly with energy and then with a relatively steeper rate (around $E \gtrsim 9 \mathrm{GeV}$ ). This is due to the additive contribution of the last term in eq. (5.1) when $\theta_{13}^{m}$ increases rapidly with energy. In the top left panel, presence of $\beta$ or $\gamma$ shows a similar change in $\Delta m_{31, m}^{2}$ while the introduction of $\varepsilon_{\mu \tau}$ shows a steady and almost linear increase 


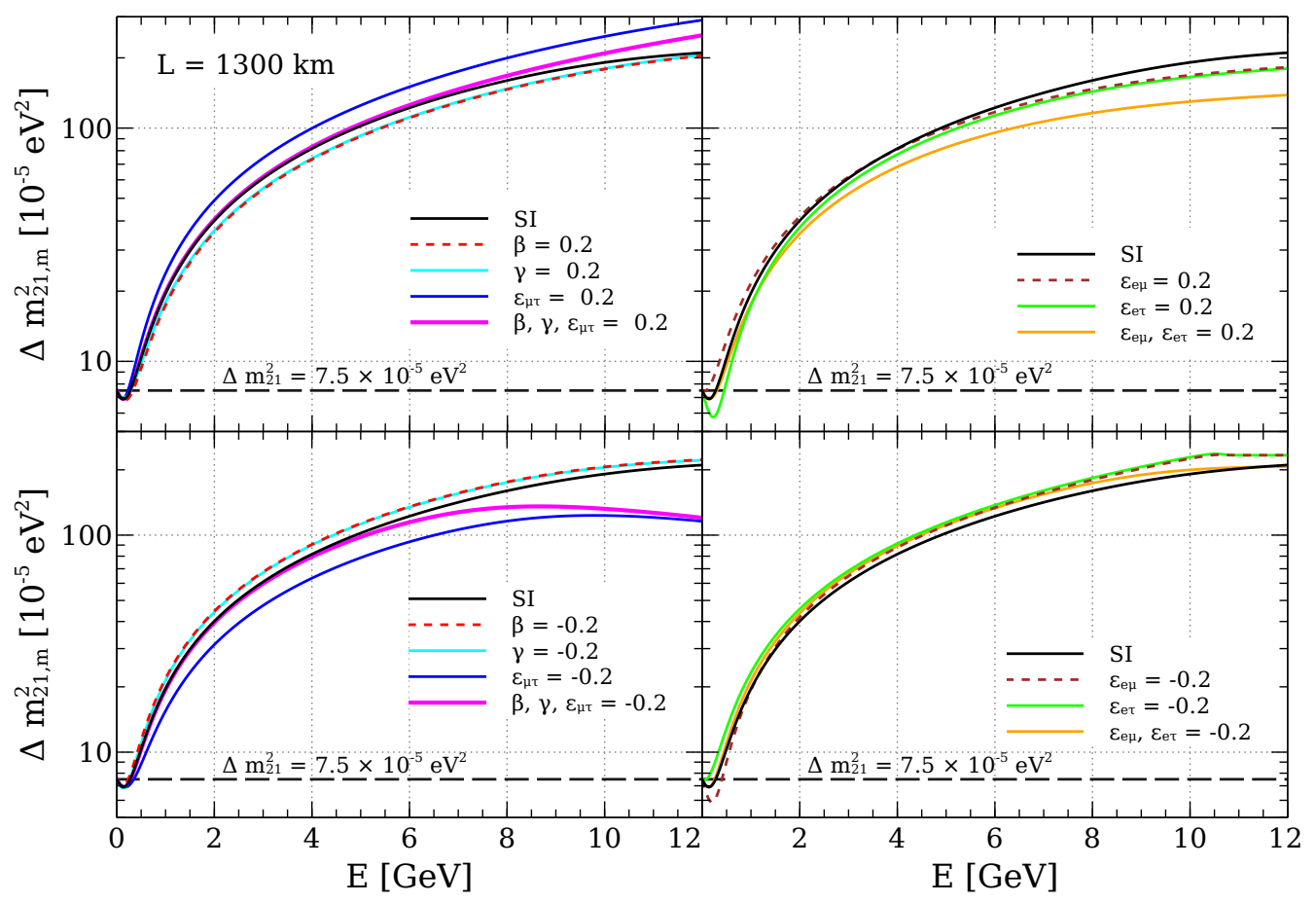

Figure 7. Variation of $\Delta m_{21, m}^{2}\left(\equiv m_{2, m}^{2}-m_{1, m}^{2}\right)$ as obtained from eqs. (5.1)-(5.3) is shown with energy in SI case and SI+NSI cases. Top (bottom) row corresponds to the positive (negative) NSI with strength 0.2. The solid black curve in each panel shows the SI case while the other curves show the modification in presence of NSI. The left column depicts the presence of various NSI parameters in $(2,3)$ block while the right shows the effect of $\varepsilon_{e \mu}$ and $\varepsilon_{e \tau}$. We consider $L=1300 \mathrm{~km}$ and the values of the oscillation parameters used in this plot are taken from table 2 . We assume $\theta_{23}=45^{\circ}$ and NMO.

with energy due to the increase of $\lambda_{3}$ appearing in r.h.s. of eq. (5.1). In the top right panel, the presence of $\varepsilon_{e \mu}$ or $\varepsilon_{e \tau}$ shows identical effects and makes $\Delta m_{31, m}^{2}$ rise with a steeper rate. Both $\varepsilon_{e \mu}$ and $\varepsilon_{e \tau}$ when present together generate an additive effect and further elevates the steepness of $\Delta m_{31, m}^{2}$. In the bottom row, we show the modification in the presence of negative NSI with strength 0.2. Presence of $\beta$ or $\gamma$ with flipped signs reverse the behavior of $\Delta m_{31, m}^{2}$. In presence of negative $\varepsilon_{\mu \tau}$, initially, there is a steady decrease in the value of $\Delta m_{31, m}^{2}$ because of the decreasing behavior of $\lambda_{3}$. However, at higher energy $(E \gtrsim 7 \mathrm{GeV})$, we see sudden growth in the evolution of $\Delta m_{31, m}^{2}$ due to increase in the value of $\theta_{13}^{m}$ at a faster rate which in turn increase the value of $m_{3, m}^{2}$. In the bottom right panel, we show the evolution in the presence of $\varepsilon_{e \mu}$ and/or $\varepsilon_{e \tau}$ with negative strength. In the presence of negative $\varepsilon_{e \mu}$ or $\varepsilon_{e \tau}$, the value of $\Delta m_{31, m}^{2}$ becomes almost constant initially $(E \lesssim 10.5 \mathrm{GeV}$ ), which can be understood from the variation of $\theta_{13}^{m}$ in the presence of negative NSI (bottom right panel of figure 4) and the fact that $\lambda_{3}$ is constant in the presence of $\varepsilon_{e \mu}$ or $\varepsilon_{e \tau}$. At $E \gtrsim$ $10 \mathrm{GeV}$, a sudden increase in the value of $\theta_{13}^{m}$ leads to the increasing behavior of $\Delta m_{31, m}^{2}$ around that energy.

In figure 7, we have shown the evolution of $\Delta m_{21, m}^{2}$ with energy for the baseline $1300 \mathrm{~km}$ and $\theta_{23}=45^{\circ}$. The black curve in each panel corresponds to the SI case while other curves 
show the evolution in presence of NSI. Top (Bottom) row corresponds to the modification in the presence of positive (negative) NSI with strength 0.2. SI case shows steady increase with energy, - reaching a value of an order as high as $\gtrsim 10^{-3} \mathrm{eV}^{2}$ from its vacuum order of magnitude $10^{-5} \mathrm{eV}^{2}$. In other words, the variation of $\Delta m_{21, m}^{2}$ can make itself comparable in magnitude with that of $\Delta m_{31, m}^{2}$. In the presence of positive (top row) or negative (bottom row) NSI (except for negative $\varepsilon_{\mu \tau}$ or taking negative $\beta, \gamma, \varepsilon_{\mu \tau}$ together), the behavior of $\Delta m_{21, m}^{2}$ does not show significant deviation in magnitude from SI case. But interestingly, depending on the sign of NSI parameter, the magnitude of $\Delta m_{21, m}^{2}$ in presence of NSI is slightly higher or lower than in presence of SI. In presence of negative $\varepsilon_{\mu \tau}$ (when present singly or together with negative $\beta$ and $\gamma$ ), we see a deviation from SI case at higher energy which can be understood from the variation of $\lambda_{1}$ with energy. With negative $\varepsilon_{e \mu}$ or $\varepsilon_{e \tau}$, however, at $E \gtrsim 10.5 \mathrm{GeV} \Delta m_{21, m}^{2}$ becomes almost constant. It happens due to a sudden increase in the value of $\theta_{13}^{m}$ around that energy which results in saturation of the value of $\lambda_{1}$.

In the case of IMO, evolution of $\Delta m_{21, m}^{2}$ is almost the same as ( $\left.\nu, \mathrm{NMO}\right)$ case for both neutrino and antineutrino propagation. However, IMO leads to a significant change in the evolution of $\Delta m_{31, m}^{2}$ for both neutrino and antineutrino propagation which is obvious because the vacuum value $\Delta m_{31}^{2}$ changes its sign.

\section{$6 \quad \theta_{13}$-resonance in the presence of NSI}

From the evolution of $\theta_{13}^{m}$ (eq. (4.2)), we see that interestingly there exists a resonance such that

$$
\hat{A}=\lambda_{3}-\alpha s_{12}^{2} c_{13}^{2}-s_{13}^{2} .
$$

Consequently, the denominator of the r.h.s. of eq. (4.2) becomes close to zero and $\theta_{13}^{m}$ becomes maximal $\left(45^{\circ}\right)$. We note that this resonance is independent of the value of $\varepsilon_{e \mu}$ or $\varepsilon_{e \tau}$ (as evident from the right panels of figure 4) but depends upon NSI parameters in the $(2,3)$ block. We know that for the SI case, under the one mass scale dominance (OMSD) approximation $\left(\Delta m_{31}^{2} L / 4 E \gg \Delta m_{21}^{2} L / 4 E\right)$, the resonance occurs at an energy $E_{\text {res }}$ such that [57],

$$
\left[E_{\mathrm{res}}^{\mathrm{SI}}\right]_{\mathrm{OMSD}}=\frac{\Delta m_{31}^{2} \cos 2 \theta_{13}}{2 V_{C C}},
$$

where, $V_{C C}$ is the standard $W$-exchange interaction potential in matter (eq. (2.7)). In presence of NSI, we seek to find out the modifications in eq. (6.2) considering $\theta_{23}=45^{\circ}$. After replacing $\cos 2 \theta_{23}^{m}$ from eq. (3.9) in the expression for $\lambda_{3}$ (eq. (3.12)), we obtain,

$$
\begin{aligned}
\lambda_{3} \simeq \frac{1}{2} & {\left[c_{13}^{2}+\alpha c_{12}^{2}+\alpha s_{12}^{2} s_{13}^{2}+(\beta+\gamma) \hat{A}\right.} \\
& \left.+\sqrt{\left\{\alpha s_{13} \sin 2 \theta_{12}+(\gamma-\beta) \hat{A}\right\}^{2}+\left\{c_{13}^{2}-\alpha c_{12}^{2}+\alpha s_{12}^{2} s_{13}^{2}+2 \varepsilon_{\mu \tau} \hat{A}\right\}^{2}}\right] .
\end{aligned}
$$

In the above equation, we neglect the small terms proportional to $\alpha s_{13}^{2},(\gamma-\beta)^{2} \hat{A}^{2}$ and the cross-term proportional to $\alpha \hat{A}(\gamma-\beta) s_{13}$. Finally, we get the following simpler expression 
for $\lambda_{3}$,

$$
\lambda_{3} \simeq c_{13}^{2}+\frac{1}{2}\left(\beta+\gamma+2 \varepsilon_{\mu \tau}\right) \hat{A} .
$$

It is noteworthy to mention that for SI case, we get $\lambda_{3} \simeq c_{13}^{2}$. Putting this back in eq. (6.1) and using OMSD approximation, we easily obtain the well-known expression for resonance in eq. (6.2). Equating eqs. (6.1) and (6.4), we obtain the following final expression for the resonance energy,

$$
E_{\mathrm{res}}^{\mathrm{NSI}} \simeq \frac{\Delta m_{31}^{2} \cos 2 \theta_{13}}{2 V_{C C}}\left[\frac{1-\left(\alpha s_{12}^{2} c_{13}^{2} / \cos 2 \theta_{13}\right)}{1-\frac{1}{2}\left(\beta+\gamma+2 \varepsilon_{\mu \tau}\right)}\right]=\left[E_{\mathrm{res}}^{\mathrm{SI}}\right]_{\mathrm{OMSD}}\left[\frac{1-\left(\alpha s_{12}^{2} c_{13}^{2} / \cos 2 \theta_{13}\right)}{1-\frac{1}{2}\left(\beta+\gamma+2 \varepsilon_{\mu \tau}\right)}\right] .
$$

The term in the square bracket in the r.h.s. of eq. (6.5) is the correction over eq. (6.2). The term $\frac{1}{2}\left(\beta+\gamma+2 \varepsilon_{\mu \tau}\right)$ is the correction induced by the presence of NSI, while $\alpha s_{12}^{2} c_{13}^{2} / \cos 2 \theta_{13}$ is the modification induced by relaxing the OMSD approximation. Thus it is now also clear analytically that $\theta_{13}^{m}$-resonance gets affected only by the NSI parameters in the $(2,3)$ block and not by $\varepsilon_{e \mu}$ or $\varepsilon_{e \tau}$.

In figure 8 , we plot the $\theta_{13}$-resonance energy as a function of the matter density $(\rho)$ inside the Earth. The matter density $(\rho)$ inside the Earth typically varies in the range of $2 \mathrm{~g} / \mathrm{cm}^{3}$ to $14 \mathrm{~g} / \mathrm{cm}^{3}$ as we move towards the center of Earth from the surface. The four grey shaded regions with varying intensity in the figure show the four different layers inside the Earth considered in this work, namely, crust, mantle, outer core, and inner core. The densities in these layers are taken from the so-called PREM profile of the Earth [99]. From eq. (6.5), we observe that for each value of the matter density, ${ }^{12}$ there will be a different value of the $\theta_{13}$-resonance energy in SI case as well as in the presence of NSI. The solid black curve shows the SI case, for which the value of the $\theta_{13}$-resonance energy decreases with the increase in the matter density. In other words, resonance energy decreases as neutrino travels deep inside the Earth. Note that there is a sudden decrease in the $\theta_{13}$-resonance energy at the boundary of mantle and outer core. Also, we observe a slight decrease in resonance energy as neutrinos enter into the inner core from the outer core. It happens due to a sudden jump in the matter density at the boundaries of these layers. Similar trends are observed in SI+NSI cases as shown by other colored curves (considered one NSI parameter at-a-time as shown in the legend). However, the value of the $\theta_{13}$-resonance energy increases (decreases) compared to the SI case at a given matter density for the positive (negative) value of the NSI parameter, as shown by the dot-dashed (dashed) curve.

\section{Impact of NSI in $\nu_{\mu}-\nu_{e}$ appearance channel}

One of the most important oscillation channel that is probed in LBL experiment is $\nu_{\mu} \rightarrow \nu_{e}$ appearance channel. This channel will play a significant role in determining the value of the CP phase, neutrino mass ordering, octant of $\theta_{23}$ from various upcoming neutrino oscillation experiments. So, in this section, we are interested in studying the effect of NSI on

\footnotetext{
${ }^{12}$ Earth matter potential $\left(V_{C C}\right)$ and matter density $(\rho)$ are connected through eq. (2.7).
} 


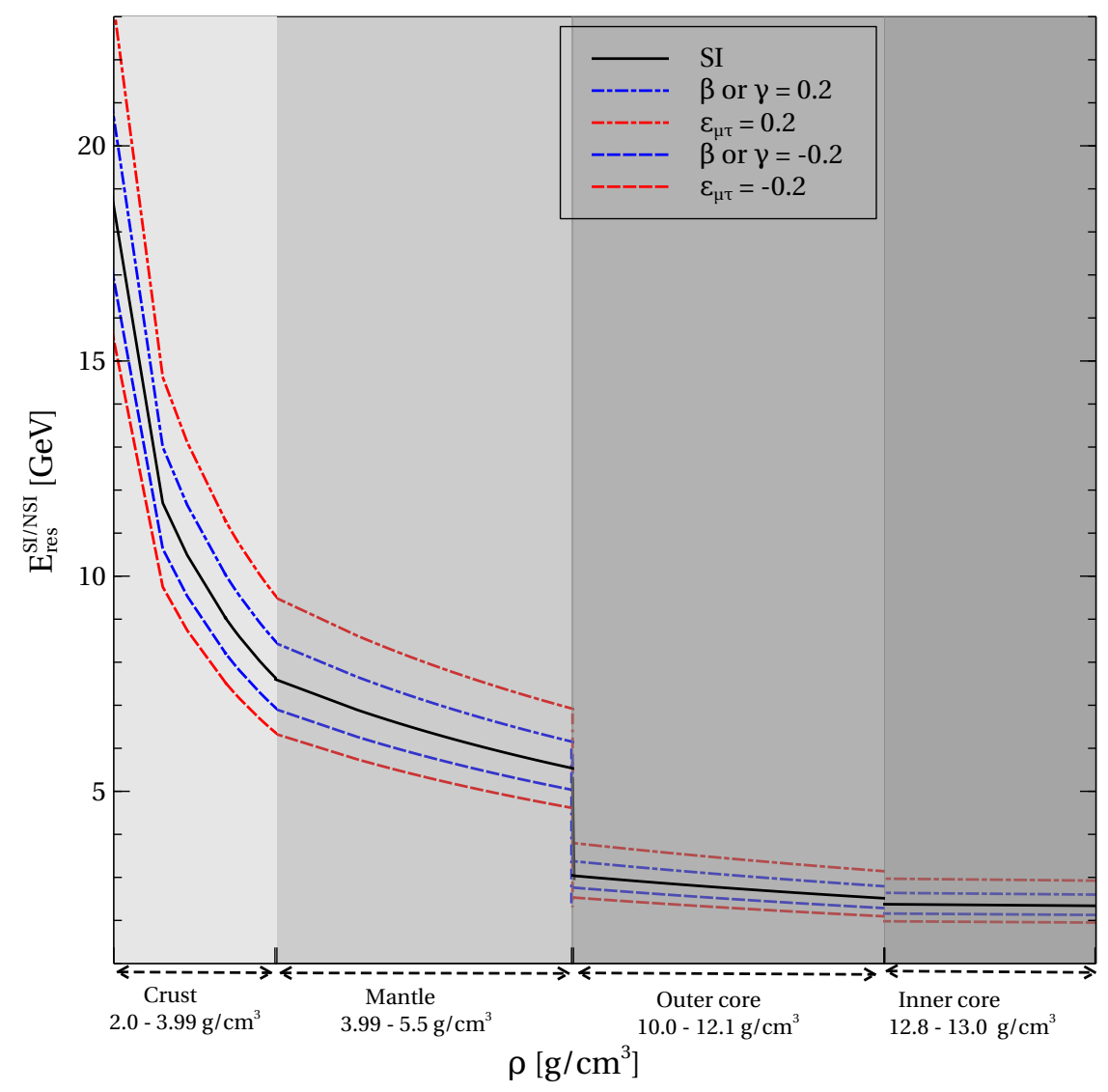

Figure 8. $\theta_{13}$-resonance energy (see eq. (6.5)) as a function of the matter density $\rho$ inside the Earth. The solid black line shows the SI case. The dot-dashed (dashed) lines correspond positive (negative) values of the NSI parameters considering one NSI parameter at-a-time, as shown in the legend. The four grey shaded regions with varying intensity show the four different layers (crust, mantle, outer core, and inner core) and their corresponding densities inside the Earth [99]. The values of the benchmark oscillation parameters used in this plot are taken from table 2 . We assume $\theta_{23}=45^{\circ}$ and $\mathrm{NMO}$.

$\nu_{\mu} \rightarrow \nu_{e}$ transition probability maxima at various baselines $(L)$ through the Earth-matter with neutrino beam having energy $(E)$ in the $\mathrm{GeV}$ range. In order to study this, in figure 9 , we plot the $\nu_{\mu} \rightarrow \nu_{e}$ transition probability in $(E-L)$ plane in SI case and SI+NSI cases considering a benchmark value of 0.2 for the strength of the NSI parameters. We calculate the oscillation probabilities for various baselines considering the well-known four-layered profile of the Earth, namely, crust, mantle, outer core, and inner core [100], which takes care of all the important features of Earth. We evaluate the transition amplitude matrices separately for each part of a given baseline passing through various layers inside the Earth. Then, finally, we calculate the $\nu_{\mu} \rightarrow \nu_{e}$ transition probabilities using the resultant amplitude as discussed in detail in appendix C. We check that figure 9 shows very good agreement in both SI and SI+NSI cases with the exact three-flavor oscillation probabilities which are calculated numerically using the GLoBES software [97, 98]. Top left panel shows the SI case where no NSI are taken into account. Here, it is observed that the region of maximum 


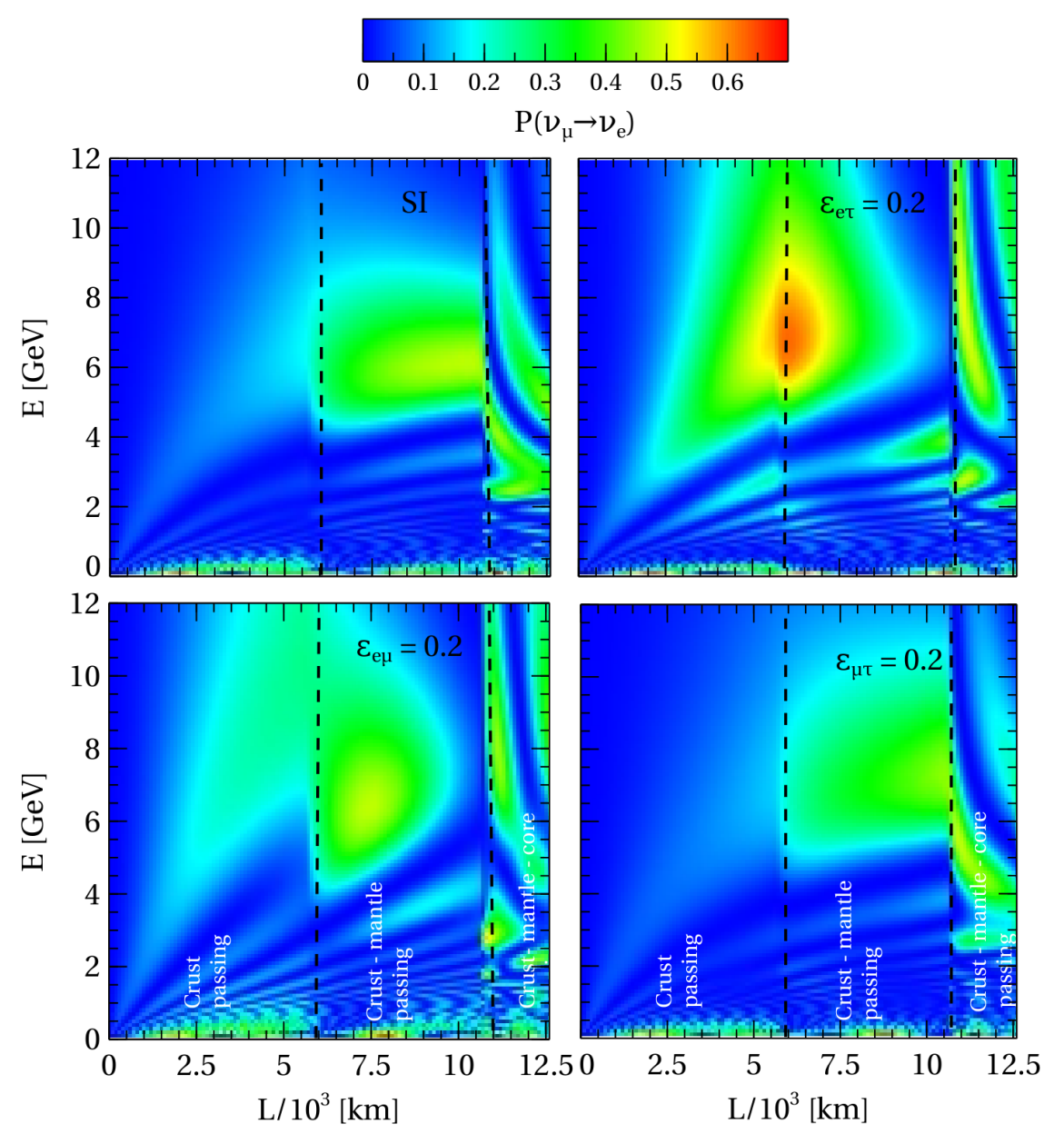

Figure 9. Oscillograms of $\nu_{\mu} \rightarrow \nu_{e}$ transition probability as a function of baseline $L$ and energy $E$. Top left panel corresponds to SI case and other three panels correspond to the cases in presence of non-zero positive NSI parameters (taken one-at-a-time with a strength of 0.2 as shown in the legends). The values of the oscillation parameters used in this plot are taken from table 2 with $\theta_{23}=45^{\circ}$ and $\mathrm{NMO}$.

appearance probability occurs for the baseline almost passing through the core and the mantle boundary. However, in presence of $\varepsilon_{e \mu}$ (bottom left panel) or $\varepsilon_{e \tau}$ (top right panel) this region shifts towards lower baselines. In case of $\varepsilon_{\mu \tau}$ (bottom right panel), this region remains almost the same as in the SI case. To show the effect of NSI with negative strength, we similarly plot the oscillograms in figure 10 in SI case and in the presence of negative NSI with strength 0.2 . Huge differences in the oscillation patterns can be observed in case of $\varepsilon_{e \mu}$ and $\varepsilon_{e \tau}$. Unlike figure 9 , there is no such region of the maximum transition probability in figure 10.

In figure 9 , we notice that for positive values of $\varepsilon_{e \mu}$ and $\varepsilon_{e \tau}$, a significant enhancement in the $\nu_{\mu} \rightarrow \nu_{e}$ transition probability as compared to SI case, for some choices of $L$ and $E$ where we have large matter effects. On the other hand, in figure 10, for negative choices of $\varepsilon_{e \mu}$ and $\varepsilon_{e \tau}$, we see a large depletion in $\nu_{\mu} \rightarrow \nu_{e}$ transition probability for some choices 


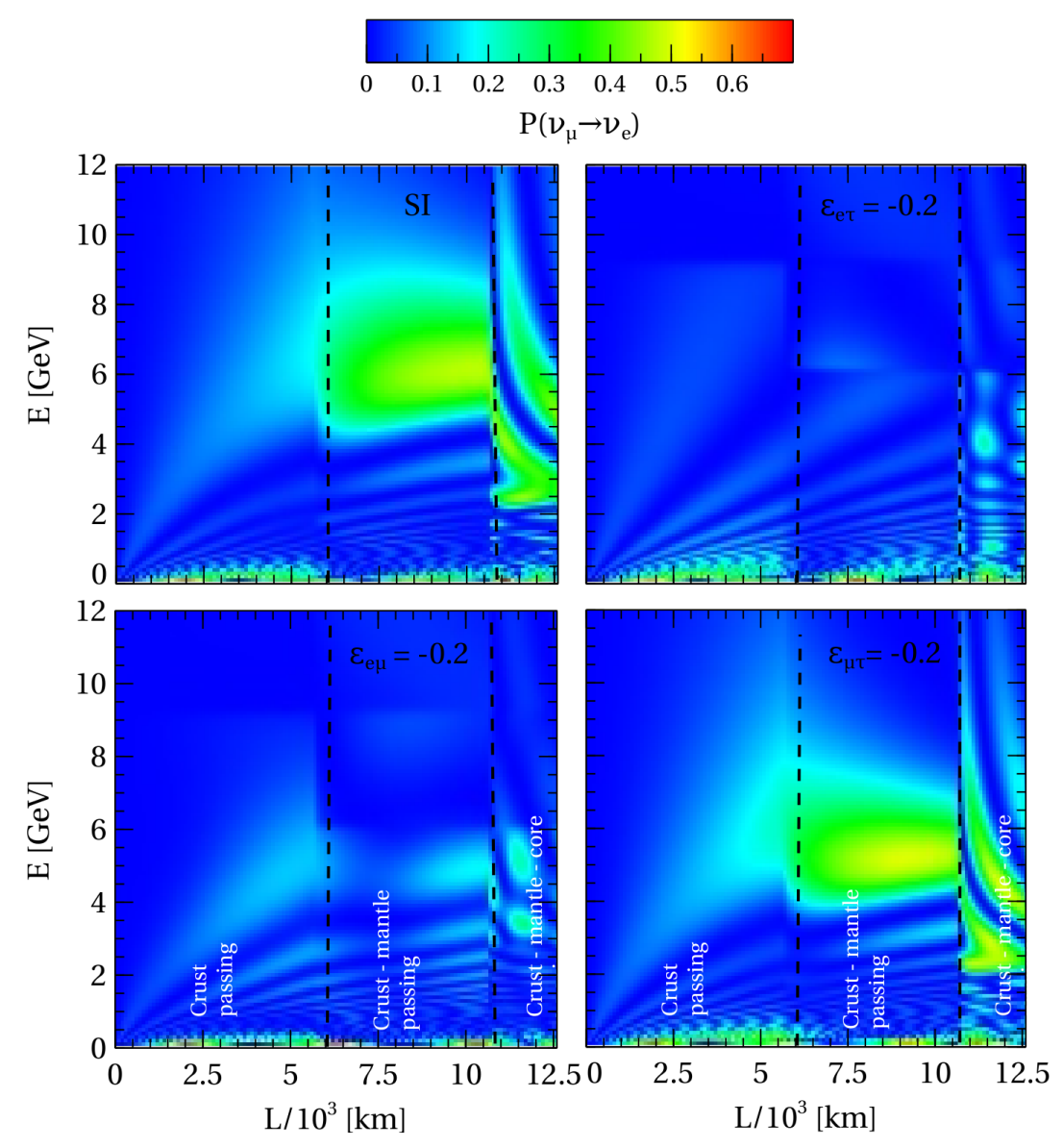

Figure 10. Oscillogram of $\nu_{\mu} \rightarrow \nu_{e}$ transition probability as a function of baseline $L$ and energy $E$. Top left panel corresponds to SI case and other three panels correspond to the cases in presence of non-zero negative NSI parameters (taken one-at-a-time with a strength of 0.2 as shown in the legends). The values of the oscillation parameters used in this plot are taken from table 2 with $\theta_{23}=45^{\circ}$ and $\mathrm{NMO}$.

of $L$ and $E$ where matter effect is suppressed. Now, we make an attempt to understand these features with the help of approximate analytical expressions. After replacing the vacuum oscillation parameters with their modified counterparts in the $\nu_{\mu} \rightarrow \nu_{e}$ transition probability as mentioned above, we simplify it further by using the approximation that $\theta_{12}^{m}$ almost saturates to $\pi / 2$ (see figure 5 and the related discussion in subsection 4.3). As a result, we obtain the following simplified expression that helps us to explain the broad features observed in figures 9 and 10 ,

$$
P_{\nu_{\mu} \rightarrow \nu_{e}}^{m}=\underbrace{\sin ^{2} \theta_{23}^{m}}_{T_{1}} \underbrace{\sin ^{2} 2 \theta_{13}^{m}}_{T_{2}} \underbrace{\sin ^{2}\left[\frac{1.27 \times \Delta m_{32, m}^{2} L}{E}\right]}_{T_{3}} .
$$

In figure 11, we plot eq. (7.1) with energy and also the contribution from each term $T_{1}$, $T_{2}$, and $T_{3}$, separately considering a baseline of $5000 \mathrm{~km}$ for which $\rho_{\text {avg }}=3.589 \mathrm{~g} / \mathrm{cm}^{3}$. It is clear from the figure that the variation of $T_{1}$ with energy is very less compared to the 

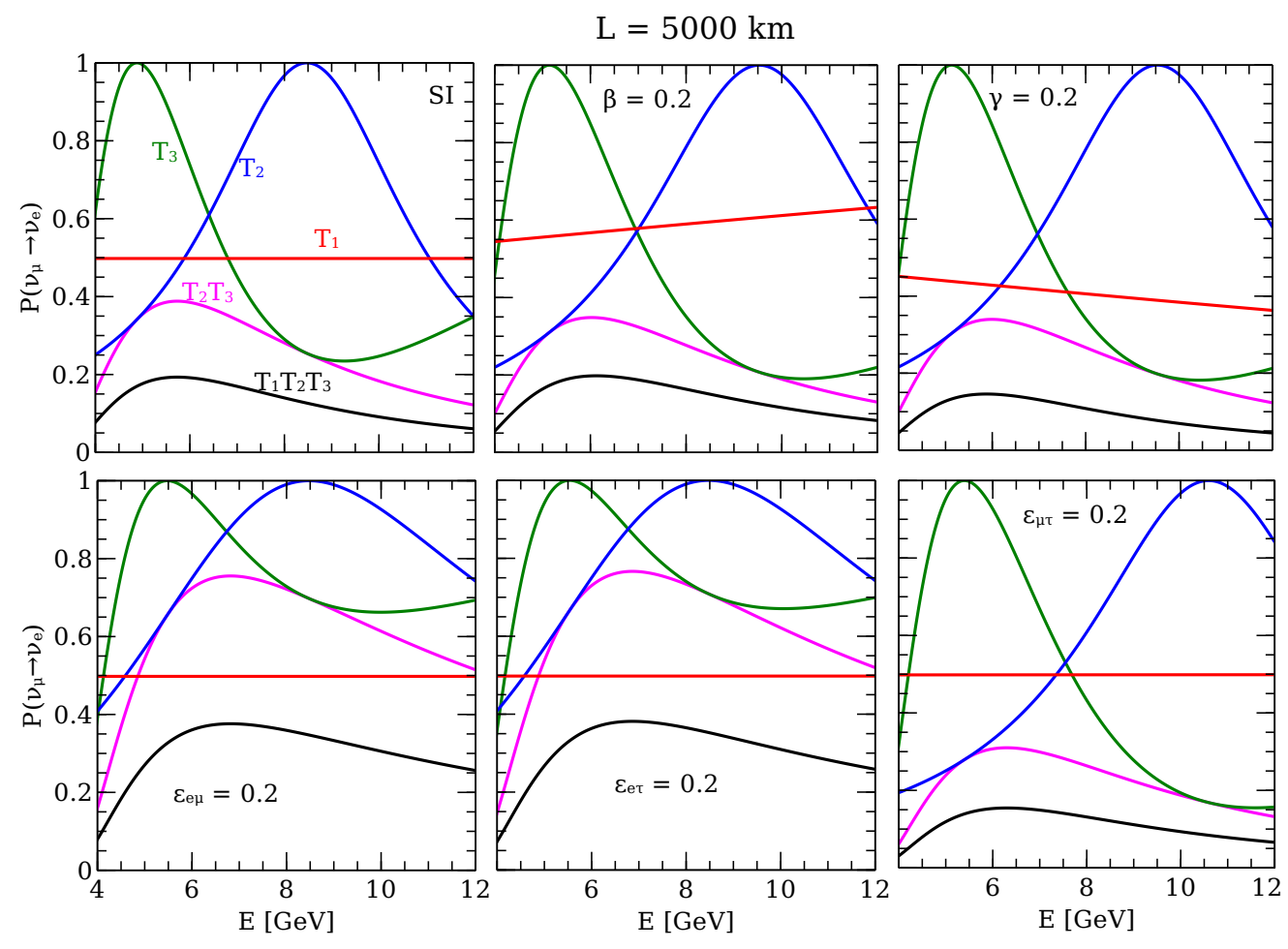

Figure 11. Variation of $\nu_{\mu} \rightarrow \nu_{e}$ transition probability (eq. (7.1)) with energy under the approximation $\theta_{12}^{m} \rightarrow 90^{\circ}$ for a baseline of $L=5000 \mathrm{~km}$. $T_{1}$ (red curve), $T_{2}$ (blue curve), and $T_{3}$ (green curve) are the three terms defined in eq. (7.1). Various panels represent the SI case and SI+NSI cases as shown in the labels. To prepare this plot, the values of the three-flavor oscillation parameters are taken from table 2. We assume $\theta_{23}=45^{\circ}$ and NMO.

variation of $T_{2}$ and $T_{3}$. Thus, the energy at which maximum of $T_{1} T_{2} T_{3}$ occurs is the same as that of $T_{2} T_{3}$. In other words, the maximum of $P_{\nu_{\mu} \rightarrow \nu_{e}}^{m}$ occurs at an energy determined by $T_{2}$ and $T_{3}$, not $T_{1}$. This feature is also valid for any other baselines. So, $P_{\nu_{\mu} \rightarrow \nu_{e}}^{m}$ is maximum when both the following two conditions are satisfied simultaneously.

- $T_{2} \equiv \sin ^{2} \theta_{13}^{m}=1$ i.e., $\theta_{13}^{m}=45^{\circ}\left(\theta_{13}\right.$-resonance condition $)$. This condition is achieved in SI case when $E=E_{\text {res }}=\frac{\Delta m_{31}^{2} \cos 2 \theta_{13}}{2 V_{C C}}$ (see eq. (6.2)) with the OMSD approximation.

- $T_{3} \equiv \sin ^{2}\left[\frac{1.27 \times \Delta m_{32, m}^{2} L}{E}\right]=1$ for some energy $E=E_{\max }^{m}$, such that

$$
E_{\max }^{m}=\frac{1.27 \times \Delta m_{32, m}^{2} L}{(2 n+1) \pi / 2} \quad \text { with } n=0,1,2 \ldots
$$

Thus, the maximum matter effect is obtained when the condition $E_{\mathrm{res}}=E_{\max }^{m}$ is satisfied [101-103].

In order to simplify the expression of $E_{\max }^{m}$ in the presence of SI only, we use eq. (5.1) and eq. (5.2) to calculate $\Delta m_{32, m}^{2}$ considering all the NSI parameters to be zero. Applying the OMSD approximation and $\theta_{23}=45^{\circ}$, we obtain

$$
\Delta m_{32, m}^{2}=\Delta m_{31}^{2} \sqrt{\left(\lambda_{3}-\hat{A}-s_{13}^{2}\right)+\sin ^{2} 2 \theta_{13}} .
$$


Now, using eq. (7.3) in the expression of $E_{\max }^{m}$ in eq. (7.2), the condition for the maximum matter effect $E_{\text {res }}=E_{\max }^{m}$ gets further simplified. Ultimately, we obtain a simple and compact relation between the baseline $(L)$ and the corresponding matter density $(\rho)$ to have the maximum $\nu_{\mu} \rightarrow \nu_{e}$ transition probability in matter

$$
(\rho \times L)_{\mathrm{SI}}=\frac{(2 n+1) \times \pi \times 5.18 \times 10^{3}}{\tan 2 \theta_{13}} \mathrm{~km} \mathrm{~g} / \mathrm{cm}^{3} .
$$

Note that under the OMSD approximation, the resonance energy condition in eq. (6.1) takes a very simple form: $\left(\lambda_{3}-\hat{A}-s_{13}^{2}\right)=0$ and we make use of this expression in eq. (7.3) to obtain eq. (7.4), which exactly matches with the expression derived by the authors in ref. [102].

Now, we analyze how eq. (7.4) gets modified in the presence of NC-NSI. First, we use eqs. (3.12)-(3.14) and eqs. (5.1)-(5.3) to derive the following two expressions for $m_{3, m}^{2}$ and $m_{2, m}^{2}$ under the OMSD approximation and assuming $\theta_{23}=45^{\circ}$

$$
\begin{aligned}
& m_{3, m}^{2}=\frac{\Delta m_{31}^{2}}{2}\left[\lambda_{3}+\hat{A}+s_{13}^{2}+T\right], \\
& m_{2, m}^{2}=\frac{\Delta m_{31}^{2}}{2}\left[\lambda_{3}+\hat{A}+s_{13}^{2}-T\right],
\end{aligned}
$$

where,

$$
T=\frac{1}{\sqrt{2}} \sqrt{2\left[\lambda_{3}-\hat{A}-s_{13}^{2}\right]^{2}+\left[\sin 2 \theta_{13}\left(c_{23}^{m}+s_{23}^{m}\right)+2 \sqrt{2}\left(\varepsilon_{e \mu} s_{23}^{m}+\varepsilon_{e \tau} c_{23}^{m}\right) \hat{A}\right]^{2}} .
$$

Using the resonance energy condition (eq. (6.1)), we now have

$$
\Delta m_{32, m}^{2}=m_{3, m}^{2}-m_{2, m}^{2}=\Delta m_{31}^{2}\left[\frac{1}{\sqrt{2}} \sin 2 \theta_{13}\left(c_{23}^{m}+s_{23}^{m}\right)+2\left(\varepsilon_{e \mu} s_{23}^{m}+\varepsilon_{e \tau} c_{23}^{m}\right) \hat{A}\right] .
$$

Replacing $\Delta m_{32, m}^{2}$ in eq. (7.2), we finally have the following condition for the maximal $\nu_{\mu} \rightarrow \nu_{e}$ transition probability in the presence of NC-NSI.

$$
\begin{aligned}
& (\rho \times L)_{\mathrm{NSI}} \\
& \simeq \frac{(2 n+1) \pi \times 5.18 \times 10^{3}}{\tan 2 \theta_{13}\left[\left\{1-\frac{1}{2}\left(\beta+\gamma+2 \varepsilon_{\mu \tau}\right)\right\}\left(\frac{c_{23}^{m}+s_{23}^{m}}{\sqrt{2}}\right)+\left\{2\left(\varepsilon_{e \mu} s_{23}^{m}+\varepsilon_{e \tau} c_{23}^{m}\right) / \tan 2 \theta_{13}\right\}\right]} \mathrm{km} \mathrm{g/ \textrm {cm } ^ { 3 }} \\
& =(\rho \times L)_{\mathrm{SI}}\left[\frac{1}{\left\{1-\frac{1}{2}\left(\beta+\gamma+2 \varepsilon_{\mu \tau}\right)\right\}\left(\frac{c_{23}^{m}+s_{23}^{m}}{\sqrt{2}}\right)+\left\{2\left(\varepsilon_{e \mu} s_{23}^{m}+\varepsilon_{e \tau} c_{23}^{m}\right) / \tan 2 \theta_{13}\right\}}\right] \mathrm{km} \mathrm{g} / \mathrm{cm}^{3} .
\end{aligned}
$$

The second factor in the r.h.s. of eq. (7.8) is the correction introduced by the NSI parameters. As shown in figure 11, the modified $\theta_{23}^{m}$ does not run significantly and is also close to $45^{\circ}$ for maximal mixing of $\theta_{23}$. Using the approximation $\theta_{23}^{m} \simeq 45^{\circ}$, we simplify eq. (7.8) to the following

$$
(\rho \times L)_{\mathrm{NSI}}^{\max } \simeq(\rho \times L)_{\mathrm{SI}}\left[\frac{1}{1-\left(\beta+\gamma+2 \varepsilon_{\mu \tau}\right) / 2+\sqrt{2}\left(\varepsilon_{e \mu}+\varepsilon_{e \tau}\right) / \tan 2 \theta_{13}}\right] \mathrm{km} \mathrm{g} / \mathrm{cm}^{3} .
$$




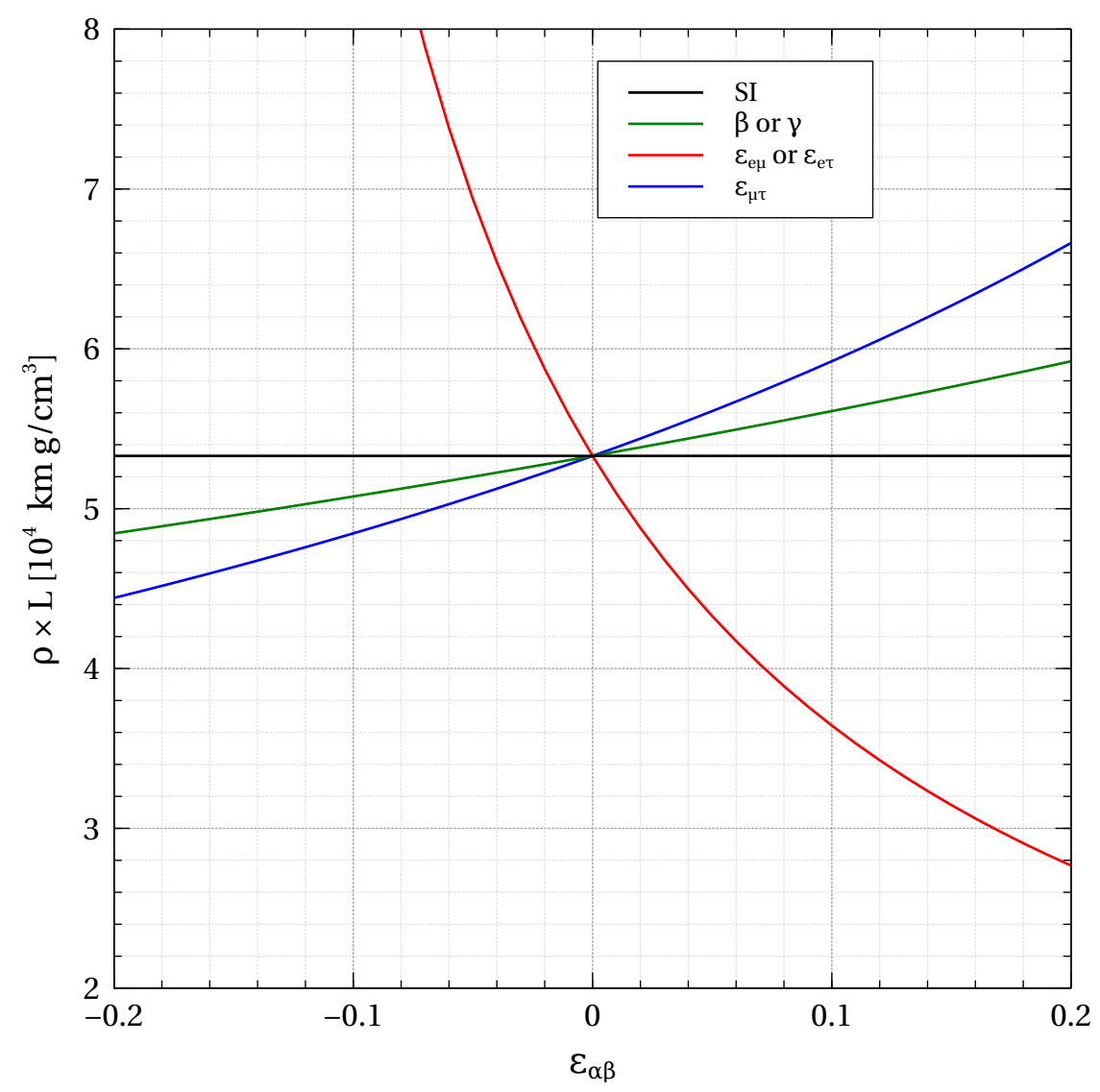

Figure 12. The product of the baseline $(L)$ and the corresponding matter density $(\rho)$ for which $\nu_{\mu} \rightarrow \nu_{e}$ transition probability attains the maximum value (see eq. (7.10)) as a function of various NSI parameters having strength in the range $[-0.2: 0.2]$. The solid black curve shows the SI case while the other colored curves correspond to the cases considering one NSI parameter at-a-time, as shown in the legend. The three-flavor oscillation parameters in vacuum are taken from table 2 with a choice of $\theta_{23}=45^{\circ}$ and $\mathrm{NMO}$.

In figure 12, we plot above equation as a function of the strength of various NSI parameters in the range of -0.2 to 0.2 . In the y-axis, we show the value of $(\rho \times L)$ for which $\nu_{\mu} \rightarrow \nu_{e}$ transition probability attains the maximum value as given in eq. (7.10). The horizontal solid black line indicates the value of the $(\rho \times L)$ for which $\nu_{\mu} \rightarrow \nu_{e}$ transition probability is maximum in the SI case. Other colored curves correspond to the SI+NSI cases considering one NSI parameter at-a-time. The green and blue curves correspond to the NSI parameters from the $(2,3)$ block, namely, $\beta$ (or $\gamma$ ) and $\varepsilon_{\mu \tau}$, respectively. In the presence of these NSI parameters, we observe a small increase or decrease in the value of $(\rho \times L)$ as compared to the SI case depending on the sign of the given NSI parameter. However, in the presence of $\varepsilon_{e \mu}$ or $\varepsilon_{e \tau}$ (as shown by the red curve), there is a significant change in the required value of $(\rho \times L)$ to attain the maximum $\nu_{\mu} \rightarrow \nu_{e}$ transition probability as compared to the SI case. When $\varepsilon_{e \mu}$ or $\varepsilon_{e \tau}$ is positive, the required value of $(\rho \times L)$ is much smaller as compared to the SI case. In other words, when $\varepsilon_{e \mu}$ or $\varepsilon_{e \tau}$ are positive, baseline length and corresponding matter density required to achieve maximum $\nu_{\mu} \rightarrow \nu_{e}$ transition 
is much smaller than the corresponding values in SI case. We observe similar feature in figure 9 , where we plot the exact oscillation probabilities in $L$ and $E$ plane. Figure 9 clearly shows that in the presence of positive $\varepsilon_{e \mu}$ or $\varepsilon_{e \tau}$, maximum $\nu_{\mu} \rightarrow \nu_{e}$ transition regions shift towards the shorter baseline as compared to the SI case. Similarly, when the strength of $\varepsilon_{e \mu}$ or $\varepsilon_{e \tau}$ is negative, the required value of $(\rho \times L)$ is much larger than that of the SI case. When the value of $\varepsilon_{e \mu}$ (or $\varepsilon_{e \tau}$ ) is around -0.2 , it is possible that the required value of $L$ and corresponding $\rho$ to obtain maximum $\nu_{\mu} \rightarrow \nu_{e}$ transition is so large that it is not attainable inside the Earth. For this reason, as shown in figure 10, we do not observe any maximum $\nu_{\mu} \rightarrow \nu_{e}$ transition inside the Earth when the value of $\varepsilon_{e \mu}$ or $\varepsilon_{e \tau}$ is -0.2 .

It is evident from eq. (7.10) that since the role of $\varepsilon_{e \mu}$ and $\varepsilon_{e \tau}$ are on the same footing, the presence of $\varepsilon_{e \mu}$ induces an effect identical to that of $\varepsilon_{e \tau}$ with the same magnitude. But the oscillograms (figures 9 and 10) for $\varepsilon_{e \mu}$ and $\varepsilon_{e \tau}$ look quite different. This is because of the fact that $\theta_{12}^{m}$ saturates to a value higher or lower than $90^{\circ}$ in the presence of $\varepsilon_{e \mu}$ or $\varepsilon_{e \tau}$ (see figure 5). Since $\theta_{12}^{m}$ is not exactly $90^{\circ}$, we have non-zero contributions from some other terms in $\nu_{\mu} \rightarrow \nu_{e}$ oscillation probability expression, which affect the oscillograms in the presence of $\varepsilon_{e \mu}$ and $\varepsilon_{e \tau}$ in a different fashion. In the presence of negative $\varepsilon_{e \mu}$ and $\varepsilon_{e \tau}$, we observe from figure 10 that we no longer achieve the maximum transition in $\nu_{\mu} \rightarrow \nu_{e}$ oscillation channel. It is because of the fact that in this case, the baseline length required for the maximum $\nu_{\mu} \rightarrow \nu_{e}$ appearance probability turns out to be longer than the Earth's diameter (see eq. (7.10)). Therefore, it is not possible to attain the maximum $\nu_{\mu} \rightarrow \nu_{e}$ transition inside the Earth for negative values of $\varepsilon_{e \mu}$ and $\varepsilon_{e \tau}$ as evident from figure 10. We observe from figure 9 and figure 10 that in the presence of non-zero $\varepsilon_{\mu \tau}$, there are slight changes in $L$ and $E$ as compared to SI case for which we obtain maximum possible $\nu_{\mu} \rightarrow \nu_{e}$ transition.

\section{Impact of NSI in $\nu_{\mu} \rightarrow \nu_{\mu}$ disappearance channel}

So far, we have focused on $\nu_{\mu} \rightarrow \nu_{e}$ appearance channel which is one of the most important channel probed in LBL experiments. However, another crucial channel, $\nu_{\mu} \rightarrow \nu_{\mu}$ disappearance channel can be probed in LBL and atmospheric neutrino experiments. This channel can play an important role in precision measurement of the atmospheric oscillation parameters. In this section, we discuss the effect of NSI in $\nu_{\mu} \rightarrow \nu_{\mu}$ survival probability. Since NSI parameters from the $(2,3)$ block have significant impact on this channel $[68,104]$, only these NSI parameters have been considered. To get the broad feature, we simplify the analysis by assuming $\Delta_{21} \simeq 0$ and $\theta_{13} \simeq 0$. Under these approximations, $\nu_{\mu} \rightarrow \nu_{\mu}$ disappearance probability expression reduces to $[105,106]$

$$
P_{\nu_{\mu} \rightarrow \nu_{\mu}}=1-\sin ^{2} 2 \theta_{23} \sin ^{2}\left[\frac{\Delta m_{31}^{2} L}{4 E}\right] .
$$

Now, we replace the vacuum oscillation parameters by the corresponding modified parameters in the presence of SI and NC-NSI assuming the line-averaged constant Earth matter density. 
Thus, eq. (8.1) takes the form:

$$
P_{\nu_{\mu} \rightarrow \nu_{\mu}}^{m}=1-\sin ^{2} 2 \theta_{23}^{m} \sin ^{2}\left[\frac{\Delta m_{31, m}^{2} L}{4 E}\right] .
$$

Using OMSD approximation $\left(\Delta m_{31}^{2} L / 4 E \gg \Delta m_{21}^{2} L / 4 E\right)$ and $\theta_{13} \simeq 0$ in eq. (3.9), also implementing $\theta_{23}=45^{\circ}$, we get

$$
\sin ^{2} 2 \theta_{23}^{m}=\frac{\left(1+2 \varepsilon_{\mu \tau} \hat{A}\right)^{2}}{[(\gamma-\beta) \hat{A}]^{2}+\left[1+2 \varepsilon_{\mu \tau} \hat{A}\right]^{2}} \simeq\left[1-\frac{(\gamma-\beta)^{2} \hat{A}^{2}}{\left(1+2 \varepsilon_{\mu \tau} \hat{A}\right)^{2}}\right] .
$$

To calculate $\Delta m_{31, m}^{2}\left(=m_{3, m}^{2}-m_{1, m}^{2}\right)$ in the last term of eq. (8.2), we use eq. (5.1) and eq. (5.3) and implement all the approximations. After simplification, we obtain,

$$
\Delta m_{31, m}^{2}=\Delta m_{31}^{2}\left[\lambda_{3}-\lambda_{2}\right] \simeq \Delta m_{31}^{2}\left[1+2 \varepsilon_{\mu \tau} \hat{A}+\frac{1}{2} \frac{(\gamma-\beta)^{2} \hat{A}^{2}}{\left(1+2 \varepsilon_{\mu \tau} \hat{A}\right)}\right]
$$

where, we use the approximation $\theta_{12}^{m} \rightarrow \pi / 2$ in the expression of $m_{1, m}^{2}$ in eq. (5.3). So, using eqs. (8.3) and (8.4), $\nu_{\mu} \rightarrow \nu_{\mu}$ disappearance probability in presence of NSI parameters from $(2,3)$ sector can be written as,

$$
\begin{aligned}
& P_{\nu_{\mu} \rightarrow \nu_{\mu}}^{m}=1- {\left[1-\frac{(\gamma-\beta)^{2} \hat{A}^{2}}{\left(1+2 \varepsilon_{\mu \tau} \hat{A}\right)^{2}}\right] \times \sin ^{2}\left[\left\{1+2 \varepsilon_{\mu \tau} \hat{A}+\frac{1}{2} \frac{(\gamma-\beta)^{2} \hat{A}^{2}}{\left(1+2 \varepsilon_{\mu \tau} \hat{A}\right)}\right\} \frac{\Delta m_{31}^{2} L}{4 E}\right] } \\
&=\cos ^{2}\left[\left\{1+2 \varepsilon_{\mu \tau} \hat{A}+\frac{1}{2} \frac{(\gamma-\beta)^{2} \hat{A}^{2}}{\left(1+2 \varepsilon_{\mu \tau} \hat{A}\right)}\right\} \frac{\Delta m_{31}^{2} L}{4 E}\right] \\
&+\frac{(\gamma-\beta)^{2} \hat{A}^{2}}{\left(1+2 \varepsilon_{\mu \tau}\right)^{2}} \times \sin ^{2}\left[\left(1+2 \varepsilon_{\mu \tau} \hat{A}\right) \frac{\Delta m_{31}^{2} L}{4 E}\right] .
\end{aligned}
$$

If we only consider the off-diagonal NSI parameter $\varepsilon_{\mu \tau}$, the expression boils down to the simplified expression already derived in [105]. From the approximate expression in eq. (8.5), some broad features about the impact of NSI on the $\nu_{\mu} \rightarrow \nu_{\mu}$ survival channel can be observed. We see that the parameter $(\gamma-\beta)$ always appears in second order in eq. (8.5), while other NSI parameter $\varepsilon_{\mu \tau}$ has a linear dependence. For the same reason, the sign of $(\gamma-\beta)$, unlike the sign of $\varepsilon_{\mu \tau}$, does not affect the disappearance probability. Since the strength of NSI parameters are not very large, it is expected that the impact of $(\gamma-\beta)$ will be always small compared to $\varepsilon_{\mu \tau}$.

To find out whether these features remain intact even if we assume non-zero $\theta_{13}$ and finite $\Delta m_{21}^{2}$, in figure 13, we plot $\nu_{\mu} \rightarrow \nu_{\mu}$ survival probability as a function of baseline (x-axis) and energy (y-axis) commonly known as oscillogram plot. We first consider the full three-flavor vacuum expression of $\nu_{\mu} \rightarrow \nu_{\mu}$ survival probability without any approximation [59] and replace the vacuum parameters with their modified expressions in matter with NSI which we derive in this work. As mentioned earlier, we calculate the oscillation probabilities for various baselines considering the well-known four-layered profile of the Earth, namely, crust, mantle, outer core, and inner core [100], which takes care of all the essential features of Earth. We evaluate the transition amplitude matrices separately for each part of a given baseline 

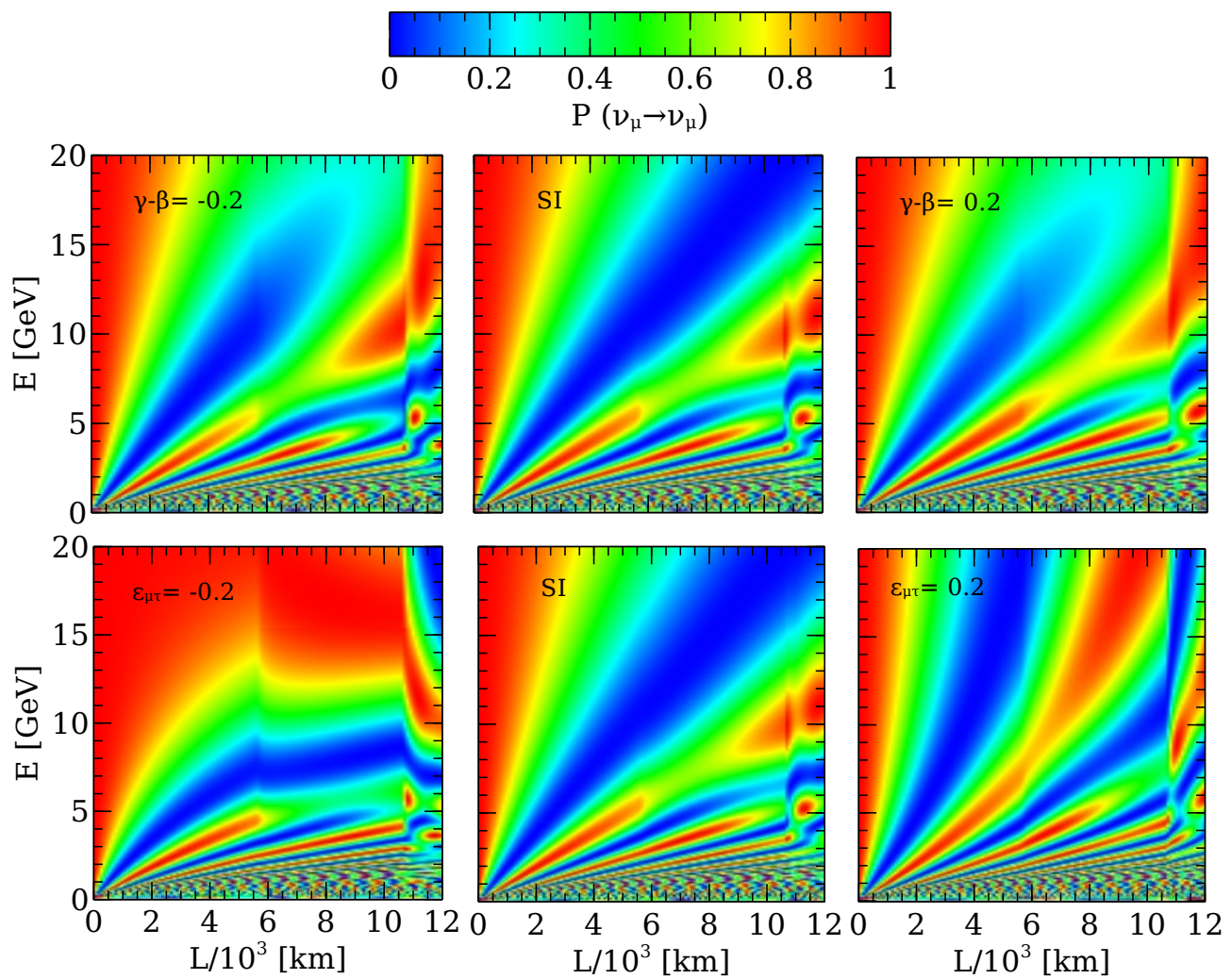

Figure 13. Oscillogram of $\nu_{\mu} \rightarrow \nu_{\mu}$ survival probability as a function of baseline $L$ and energy $E$. Top and bottom rows correspond to the oscillogram in presence of NSI parameter $(\gamma-\beta)$ and $\varepsilon_{\mu \tau}$, respectively. The middle column in both the rows shows the SI case. The first (third) column depicts the presence of negative (positive) NSI with a strength of 0.2 . Values of the oscillation parameters are taken from table 2 . We assume $\theta_{23}=45^{\circ}$ and NMO.

passing through various layers inside the Earth. Then, finally, we calculate the $\nu_{\mu} \rightarrow \nu_{\mu}$ survival probabilities using the resultant amplitude as discussed in detail in appendix C. As before, $\theta_{23}$ in vacuum is assumed to be $45^{\circ}$. In the middle panels of figure 13 , we plot $\nu_{\mu}$ survival probabilities in the SI case. Next, we compare these probabilities in the SI case with probabilities in the presence of the effective NSI parameter $(\gamma-\beta)$ considering its negative (top left panel) and positive (top right panel) values. The oscillation valley denoted by the diagonal blue shaded region (where $\nu_{\mu} \rightarrow \nu_{\tau}$ transition probability in vacuum is maximum and because of that $\nu_{\mu} \rightarrow \nu_{\mu}$ survival probability is minimum) gets diminished when effective NSI parameter $(\gamma-\beta)$ is finite we and see an enhancement in the $\nu_{\mu} \rightarrow \nu_{\mu}$ survival probabilities as compared to the SI case at higher energies and baselines. Eq. (8.5) reveals that $\nu_{\mu} \rightarrow \nu_{\mu}$ survival probability should not depend on the sign of $(\gamma-\beta)$. The top left and right panels of figure 13 confirm this fact, except for some small differences, which appear due to non-zero $\theta_{13}$, that brings the matter effect into the picture and finite value of $\Delta_{21}$ causes some differences.

In the bottom row of figure 13 , we plot the same but in presence of $\varepsilon_{\mu \tau}$ with negative (positive) value in the extreme left (right) panel and show the results for SI case in the 
middle panel. It is observed that the presence of $\varepsilon_{\mu \tau}$ can lead to significant differences in the pattern of $\nu_{\mu} \rightarrow \nu_{\mu}$ disappearance probability as compared to the SI case. When $\varepsilon_{\mu \tau}$ is positive (negative), one can observe a significant shift in the oscillation valley from the SI case towards higher (lower) energies in figure 13. This feature can be explained from the approximate expression in eq. (8.5). In that expression, the value of $P_{\nu_{\mu} \rightarrow \nu_{\mu}}^{m}$ is mainly determined by the first term in r.h.s. since second term is suppressed by the NSI parameters appearing quadratically. At the first term in r.h.s. of eq. (8.5), minimum occurs at higher (lower) energy compared to the SI case for a given baseline $L$ when $\varepsilon_{\mu \tau}$ is present with positive (negative) strength. ${ }^{13}$ Depending on the sign of $\varepsilon_{\mu \tau}$, the regions representing the oscillation dip tend to bend upward or downward with increase in baseline length compared to the SI case.

\section{$9 \quad$ Summary and concluding remarks}

In this work, we derive the expressions for the evolution of the fundamental mass-mixing parameters in the presence of SI and SI+NSI considering all possible lepton-flavor-conserving and lepton-favor-violating NC-NSI. In order to derive these expressions, we use a method of approximate diagonalization of the effective Hamiltonian by performing successive rotations in $(2,3),(1,3)$, and $(1,2)$ blocks. In our study, we present the results for the benchmark value of the DUNE baseline of $1300 \mathrm{~km}$ and also discuss the results for few other baselines. We consider both positive and negative values of real NSI parameters with benchmark values of \pm 0.2 .

In the presence of SI only, the 2-3 mixing angle in matter $\left(\theta_{23}^{m}\right)$ receives a tiny correction which is independent of energy and the strength of the matter potential. It is observed that only the NSI parameters in the $(2,3)$ block, namely $\varepsilon_{\mu \tau}$ and $(\gamma-\beta) \equiv\left(\varepsilon_{\tau \tau}-\varepsilon_{\mu \mu}\right)$ influence the evolution of $\theta_{23}^{m}$. In the presence of negative (positive) value of $(\gamma-\beta), \theta_{23}^{m}$ increases (decreases) with energy. For the maximal value of $\theta_{23}$ in vacuum, the change in $\theta_{23}^{m}$ is negligible in the presence of $\varepsilon_{\mu \tau}$. If $\theta_{23}$ belongs to the upper octant then $\theta_{23}^{m}$ increases (decreases) for negative (positive) choices of $\varepsilon_{\mu \tau}$. We notice a completely opposite behavior if $\theta_{23}$ lies in the lower octant. We also study the modification in $\theta_{23}^{m}$ as a function of energy when both the NSI parameters $\varepsilon_{\mu \tau}$ and $(\gamma-\beta)$ are present in the scenario with their all possible sign combinations. We unravel interesting degeneracies in $\left[\theta_{23}-(\gamma-\beta)\right]$ and $\left[\theta_{23}-\varepsilon_{\mu \tau}\right]$ planes for three different combination of $L$ and $E$ and discuss how our simple approximate analytical expression showing the evolution of $\theta_{23}^{m}$ plays an important role to understand these complicated degeneracy patterns.

In contrast to $\theta_{23}^{m}, \theta_{13}^{m}$ is more sensitive in matter in the presence of SI and SI+NSI. Therefore, an accurate understanding of the evolution of $\theta_{13}$ in matter is crucial to correctly assess the outcome of the oscillation experiments in the presence of NC-NSI. $\theta_{13}^{m}$ goes through an appreciable change even in SI case depending on the choice of mass ordering and whether we are dealing with neutrinos or antineutrinos. Compared to SI case, the relative change in $\theta_{13}^{m}$ for $(\nu, \mathrm{NMO})$ is somewhat suppressed (enhanced) in the presence of positive

\footnotetext{
${ }^{13}$ At oscillation dip, the argument of the cosine term in eq. (8.5) should be approximately equal to $(2 n+1) \pi / 2$ where $n=0,1,2 \ldots$ This roughly implies that $\left(1+2 \varepsilon_{\mu \tau} \hat{A}\right) \frac{\Delta m_{31}^{2} L}{4 E} \simeq \pi / 2$.
} 
(negative) NSI parameters in the $(2,3)$ block, namely $\gamma \equiv\left(\varepsilon_{\tau \tau}-\varepsilon_{e e}\right), \beta \equiv\left(\varepsilon_{\mu \mu}-\varepsilon_{e e}\right)$, and $\varepsilon_{\mu \tau}$. For positive $\varepsilon_{e \mu}$ and/or $\varepsilon_{e \tau}, \theta_{13}^{m}$ for $(\nu, \mathrm{NMO})$ approaches the resonance $\left(\theta_{13}^{m}=45^{\circ}\right)$ faster than SI case, but after crossing the resonance energy, SI takes over. For negative $\varepsilon_{e \mu}$ or $\varepsilon_{e \tau}$, modification of $\theta_{13}^{m}$ is suppressed almost up to the resonance energy and then, it increases very steeply compared to SI case.

As far as the solar mixing angle is concerned, $\theta_{12}^{m}$ approaches to $90^{\circ}\left(\sin \theta_{12}^{m} \rightarrow 1\right.$, $\left.\cos \theta_{12}^{m} \rightarrow 0\right)$ very quickly as we increase the neutrino energy in SI case. While the NSI parameters in the $(2,3)$ block $\left(\gamma, \beta, \varepsilon_{\mu \tau}\right)$ have minimal impact on the evolution of $\theta_{12}^{m}, \varepsilon_{e \mu}$ and $\varepsilon_{e \tau}$ affect the evolution of $\theta_{12}^{m}$ substantially. In the presence of positive (negative) $\varepsilon_{e \mu}$, the change in $\theta_{12}^{m}$ with energy qualitatively remains the same with the saturation value turns out to be around $80^{\circ}\left(100^{\circ}\right)$. In the presence of positive (negative) $\varepsilon_{e \tau}$, the saturation happens around $100^{\circ}\left(80^{\circ}\right)$.

Out of the two mass-squared differences, the evolution of the solar $\Delta m_{21, m}^{2}$ is quite dramatic as compared to that of the atmospheric $\Delta m_{31, m}^{2}$ in matter. Both in SI and SI+NSI cases, as we increase the energy and go beyond $10 \mathrm{GeV}$, the value of $\Delta m_{21, m}^{2}$ increases to almost 20 times as compared to its vacuum value for $1300 \mathrm{~km}$ baseline. At the same time, the value of $\Delta m_{31, m}^{2}$ does not change much compared to its vacuum value.

We demonstrate the utility of our approach in addressing some interesting features that we observe in neutrino oscillation in presence of matter. It is well known that $\theta_{13}^{m}$ can attain the value of $45^{\circ}$ (MSW-resonance condition) for some choices of $L$ and $E$ in the presence of standard matter effect. Now, in this work, for the first time, we show how the $\theta_{13}$-resonance energy gets modified in the presence of NC-NSI with the help of simple, compact, approximate analytical expressions. We observe that only the NSI parameters in the $(2,3)$ block affects the $\theta_{13}$-resonance energy.

We study in detail how the NC-NSI parameters affect $\nu_{\mu} \rightarrow \nu_{e}$ oscillation probability which plays an important role to address the remaining unknown issues, namely $\mathrm{CP}$ violation, mass ordering, and the precision measurement of oscillation parameters. In this paper, for the first time, we derive a simple approximate analytical expression for $E, L$ and its corresponding $\rho$ to have the maximal matter effect which in turn gives rise to maximum $\nu_{\mu} \rightarrow \nu_{e}$ transition in the presence of all possible NC-NSI parameters. This analytical expression (see eq. (7.10)) reveals that in SI case, maximum $\nu_{\mu} \rightarrow \nu_{e}$ transition occurs when the value of $(\rho \times L)$ is around $5.33 \times 10^{4} \mathrm{~km} \mathrm{~g} / \mathrm{cm}^{3}$ which may be satisfy for neutrinos passing through core of the Earth under the assumption that the $\theta_{13}$-resonance energy coincides with the energy that corresponds to the first oscillation maximum. However, in the presence of positive $\varepsilon_{e \mu}$ or $\varepsilon_{e \tau}$ in matter, it is observed that the required value of $(\rho \times L)$ to obtain the maximum $\nu_{\mu} \rightarrow \nu_{e}$ transition probability is much smaller as compared to the SI case (see figure 12). for an example, if the value of $\varepsilon_{e \mu} / \varepsilon_{e \tau}$ is equal to 0.1 , then one can achieve the maximum $\nu_{\mu} \rightarrow \nu_{e}$ transition for the value of $(\rho \times L)$ around $3.64 \times 10^{4} \mathrm{~km}$ $\mathrm{g} / \mathrm{cm}^{3}$, which may be realized in nature if neutrinos pass through mantle. On the other hand, if we consider negative values of $\varepsilon_{e \mu} / \varepsilon_{e \tau}$ with higher strength, the required values of $(\rho \times L)$ to attain maximum $\nu_{\mu} \rightarrow \nu_{e}$ transition may not be satisfied inside the Earth.

We also study in detail how the NSI parameters in the $(2,3)$ block affect $\nu_{\mu} \rightarrow \nu_{\mu}$ disappearance channel which plays an important role in atmospheric neutrino experiments. 
We observe that the off-diagonal NSI parameter $\varepsilon_{\mu \tau}$ has the dominant effect as compared to the diagonal NSI parameter $(\gamma-\beta)$. It happens because $(\gamma-\beta)$ appears in the second-order in the approximate $\nu_{\mu} \rightarrow \nu_{\mu}$ oscillation probability expression, whereas $\varepsilon_{\mu \tau}$ shows a liner dependence. Also, the sign of $\varepsilon_{\mu \tau}$ has a significant impact on $\nu_{\mu} \rightarrow \nu_{\mu}$ disappearance channel. However, this oscillation channel is not sensitive to the sign of the NSI parameter $(\gamma-\beta)$. We hope that the analysis performed in this paper will take our understanding of the evolution of the oscillation parameters in the presence of all possible NC-NSI a step forward.

\section{Acknowledgments}

We would like to thank T. Takeuchi, P. Denton, and E. Esteban, A. Kumar for useful discussions. S.D. is grateful to the organizers of the Neutrino 2020 Online Conference at Fermilab, Chicago, U.S.A. during 22nd June to 2nd July, 2020 for giving an opportunity to present a poster based on this work. S.D. would also like to thank the organizers of the XXIV DAE-BRNS High Energy Physics Online Symposium at NISER, Bhubaneswar, India during 14th to 18th December, 2020 for providing him an opportunity to give a talk based on this study. We thank the Department of Atomic Energy (DAE), Govt. of India for financial support. S.K.A. is supported by the INSPIRE Faculty Research Grant [IFA-PH-12] from the Department of Science and Technology (DST), Govt. of India. S.K.A. acknowledges the financial support from the Swarnajayanti Fellowship Research Grant (No. DST/SJF/PSA-05/2019-20) provided by the DST, Govt. of India and the Research Grant (File no. SB/SJF/2020-21/21) from the Science and Engineering Research Board (SERB) under the Swarnajayanti Fellowship by the DST, Govt. of India. S.K.A. and M.M. acknowledge the financial support from the Indian National Science Academy (INSA) Young Scientist Project [INSA/SP/YS/2019/269]. M.M. acknowledges the support of IBS under the project code IBS-R018-D1.

\section{A Comparison between approximate analytical expressions and exact numerical calculations at the probability level}

In section 3, we derive the expressions for the three modified mixing angles which are given in eqs. (3.9)-(3.14). Expressions for the modified mass-squared differences are shown in eqs. (5.1)-(5.3). Now one can calculate the oscillation probabilities in the presence of NC-NSI parameters in matter by replacing the vacuum oscillation parameters in the probability expressions with the corresponding modified approximate analytical expressions in matter. In this appendix, we perform a neck-to-neck comparison between various oscillation probabilities obtained using our approximate analytical expressions and full numerical results obtained from the publicly available software GLoBES. This study enables us to judge how accurate our analytical expressions are, in comparison to the exact numerical calculations at the probability level.

In figure 14, we show the comparison between the approximate analytical expressions (red curves) and exact numerical calculations (black curves) for $\nu_{\mu} \rightarrow \nu_{e}$ channel considering three different baselines $(1300 \mathrm{~km}, 5000 \mathrm{~km}$, and $8700 \mathrm{~km})$ and two different flavor-violating 

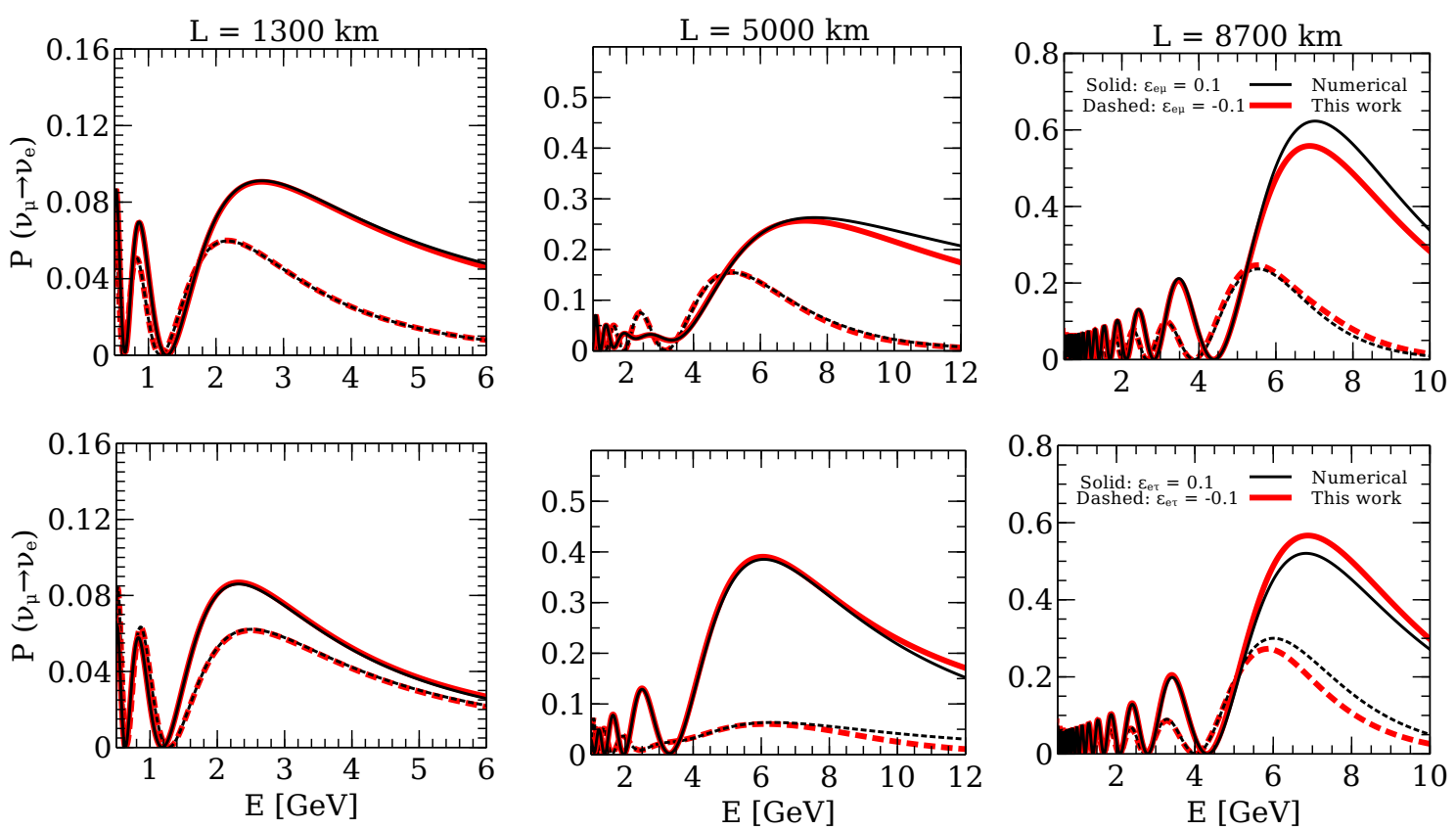

Figure 14. Comparison of $\nu_{\mu} \rightarrow \nu_{e}$ appearance probability estimated from our approximate analytical expressions (red curves), and the numerical calculations obtained from GLoBES (black curves) in the presence of NC-NSI parameters $\varepsilon_{e \mu}$ (upper panels) and $\varepsilon_{e \tau}$ (lower panels) one-at-atime. The three different columns correspond to the three different baselines: $1300 \mathrm{~km}$ (left panels), $5000 \mathrm{~km}$ (middle panels), and $8700 \mathrm{~km}$ (right panels). The solid (dashed) curves are obtained using a value of $0.1(-0.1)$ for the NSI parameters. Values of the three-flavor oscillation parameters are taken from table 2 . We assume $\theta_{23}=45^{\circ}$ and NMO.

NC-NSI parameters $\varepsilon_{e \mu}$ and $\varepsilon_{e \tau}$ (one-at-a-time). The upper (lower) panels show the results for $\varepsilon_{e \mu}\left(\varepsilon_{e \tau}\right)$. We consider both positive (solid lines) and negative (dashed lines). This plot shows that we have a very good agreement between analytical and numerical calculations.

In figure 15, we perform the same study for $\nu_{\mu} \rightarrow \nu_{\mu}$ survival channel considering the NC-NSI parameters from the $(2,3)$ block, namely, $(\gamma-\beta)$ and $\varepsilon_{\mu \tau}$ one-at-a-time. The upper panels are for $(\gamma-\beta)$ and in the lower panels we consider $\varepsilon_{\mu \tau}$. This figure also depicts a very good agreement between analytical and numerical calculations.

\section{B Evolution of mass-mixing parameters with non-zero $\delta_{\mathrm{CP}}$}

Recent global fit study of the neutrino oscillation data containing information from the solar, atmospheric, reactor, accelerator, and short-baseline experiments hint that Dirac CP-violating phase $\delta_{\mathrm{CP}}$ is non-zero in Nature with a best-fit value around $220^{\circ}$ assuming normal mass ordering [107]. Therefore, it is important to consider the non-zero values of $\delta_{\mathrm{CP}}$ in our analysis.

When $\delta_{\mathrm{CP}}$ is non-zero, some elements of the effective neutrino propagation Hamiltonian (see eq. (2.8)) matrix contain imaginary terms. In order to diagonalize the effective Hamiltonian, we apply three complex rotations: $R_{23}\left(\theta_{23}^{m}, 0\right), R_{13}\left(\theta_{13}^{m}, \delta_{\mathrm{CP}}^{m}\right)$, and $R_{12}\left(\theta_{12}^{m}, 0\right)$ successively, where $R_{i j}\left(\theta_{i j}^{m}, \phi^{m}\right)$ is the complex rotation matrix in the $(i, j)$ plane with 

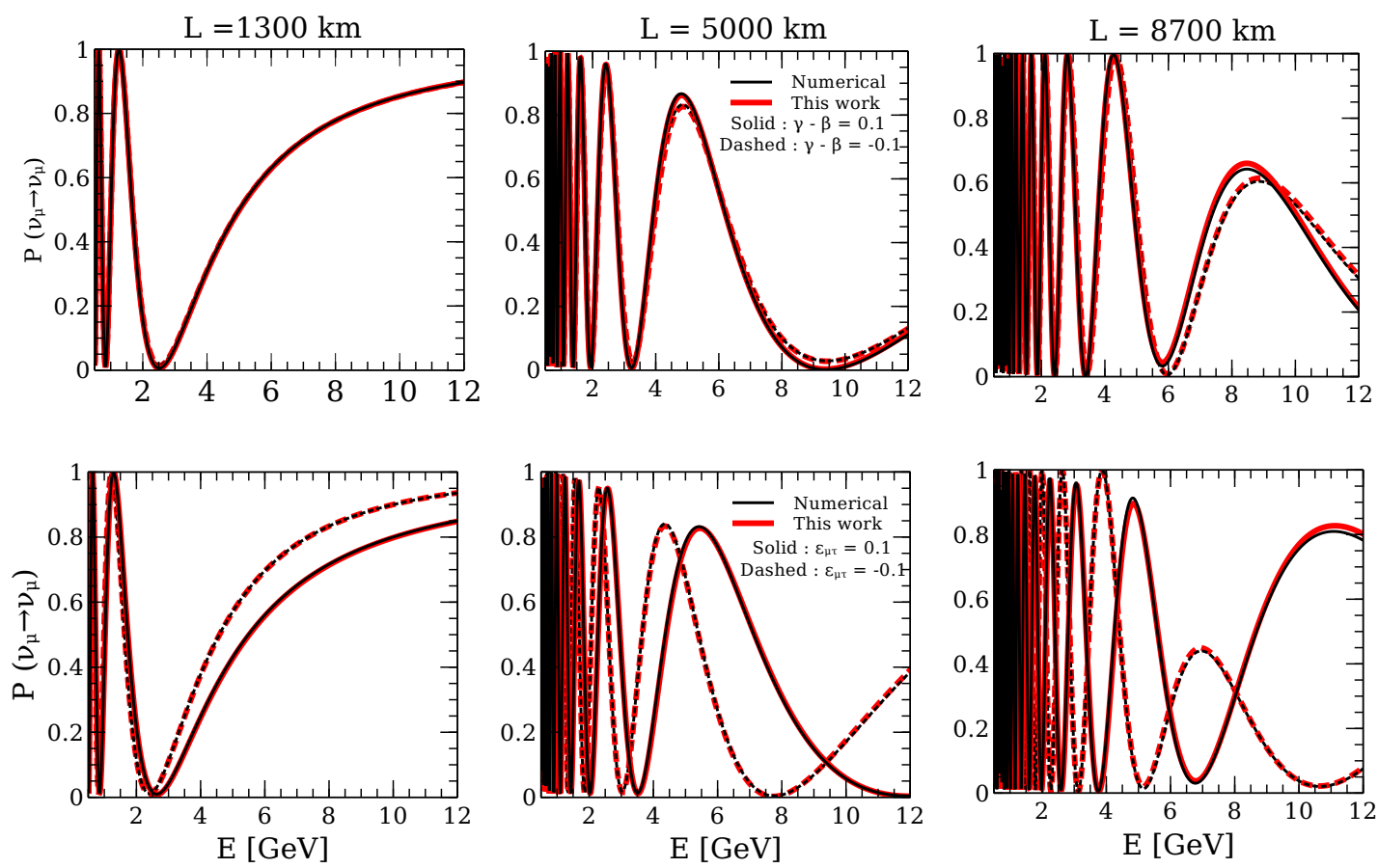

Figure 15. Comparison of $\nu_{\mu} \rightarrow \nu_{\mu}$ disappearance probability estimated from our approximate analytical expressions (red curves), and the numerical calculations obtained from GLoBES (black curves) in the presence of NC-NSI parameters $(\gamma-\beta)$ and $\varepsilon_{\mu \tau}$ one-at-a-time. In the upper panels, we consider the presence of $(\gamma-\beta)$ and lower panels are for $\varepsilon_{\mu \tau}$. The three different columns correspond to the three different baselines: $1300 \mathrm{~km}$ (left panels), $5000 \mathrm{~km}$ (middle panels), and $8700 \mathrm{~km}$ (right panels). The solid (dashed) curves are obtained using a value of $0.1(-0.1)$ for the NSI parameters. Values of the three-flavor oscillation parameters are taken from table 2. We assume $\theta_{23}=45^{\circ}$ and $\mathrm{NMO}$.

angle $\theta_{i j}^{m}$ and phase $\phi^{m}$. After performing the rotation in each plane, we separately equate the real and imaginary parts of the off-diagonal elements of that plane to zero and derive the expressions for the corresponding modified mixing angle $\left(\theta_{i j}^{m}\right)$ and phase $\left(\phi^{m}\right)$. While doing this, we neglect all the terms which are of the order $\alpha s_{13}\left(\sim 10^{-3}\right)$ or higher. ${ }^{14}$ The expressions for the modified mixing angles and CP phase are as follows

$$
\begin{aligned}
\tan 2 \theta_{23}^{m}= & \frac{c_{13}^{2}-\alpha c_{13}^{2}+2 \varepsilon_{\mu \tau} \hat{A}}{(\gamma-\beta) \hat{A}}, \\
\tan 2 \theta_{13}^{m}= & \frac{1}{\sqrt{2}\left(\lambda_{3}-\hat{A}-s_{13}^{2}-\alpha s_{12}^{2} c_{13}^{2}\right)}\left[\left\{\sin 2 \theta_{13}\left(1-\alpha s_{12}^{2}\right) \cos \delta_{\mathrm{CP}}\left(c_{23}^{m}+s_{23}^{m}\right)\right.\right. \\
& \left.-\alpha \sin 2 \theta_{12} c_{13}\left(c_{23}^{m}-s_{23}^{m}\right)+2 \sqrt{2}\left(\varepsilon_{e \mu} s_{23}^{m}+\varepsilon_{e \tau} c_{23}^{m}\right) \hat{A}\right\}^{2} \\
& \left.+\left\{\sin 2 \theta_{13}\left(1-\alpha s_{12}^{2}\right) \sin \delta_{\mathrm{CP}}\left(c_{23}^{m}+s_{23}^{m}\right)\right\}^{2}\right]^{1 / 2},
\end{aligned}
$$

\footnotetext{
${ }^{14}$ After performing the rotations in $(2,3)$ and $(1,2)$ blocks, the imaginary parts of the off-diagonal elements appear to be proportional to $\alpha s_{13}$, which is of the order of $10^{-3}$, we can safely neglect these imaginary parts of the off-diagonal elements. Note that we make this assumptions while obtaining the expressions for modified mass-mixing parameters with non-zero $\delta_{\mathrm{CP}}$, but at the probability level, we always have the terms which are of the order of $\alpha s_{13}$.
} 


$$
\begin{aligned}
\tan 2 \theta_{12}^{m}= & \frac{c_{13}^{m}}{\sqrt{2}\left(\lambda_{2}-\lambda_{1}\right)}\left[\left\{\sin 2 \theta_{13}\left(1-\alpha s_{12}^{2}\right) \cos \delta_{\mathrm{CP}}\left(c_{23}^{m}+s_{23}^{m}\right)+\alpha \sin 2 \theta_{12} c_{13}\left(c_{23}^{m}-s_{23}^{m}\right)\right.\right. \\
& \left.\left.+2 \sqrt{2}\left(\varepsilon_{e \mu} c_{23}^{m}-\varepsilon_{e \tau} s_{23}^{m}\right) \hat{A}\right\}^{2}+\left\{\sin 2 \theta_{13}\left(1-\alpha s_{12}^{2}\right) \sin \delta_{\mathrm{CP}}\left(c_{23}^{m}+s_{23}^{m}\right)\right\}^{2}\right]^{1 / 2}
\end{aligned}
$$

$\tan \delta_{\mathrm{CP}}^{m}=\tan \delta_{\mathrm{CP}}$

In the above equations, the $\lambda$ 's take the form,

$$
\begin{aligned}
& \lambda_{3}=\frac{1}{2}\left[c_{13}^{2}+\alpha c_{12}^{2}+(\beta+\gamma) \hat{A}+\sqrt{\left(c_{13}^{2}-\alpha c_{12}^{2}+2 \varepsilon_{\mu \tau} \hat{A}\right)^{2}+(\gamma-\beta)^{2} \hat{A}^{2}}\right], \\
& \lambda_{2}=\frac{1}{2}\left[c_{13}^{2}+\alpha c_{12}^{2}+(\beta+\gamma) \hat{A}-\sqrt{\left(c_{13}^{2}-\alpha c_{12}^{2}+2 \varepsilon_{\mu \tau} \hat{A}\right)^{2}+(\gamma-\beta)^{2} \hat{A}^{2}}\right], \\
& \lambda_{1}=\frac{1}{2}\left[\lambda_{3}+\hat{A}+s_{13}^{2}+\alpha s_{12}^{2} c_{13}^{2}-\frac{\lambda_{3}-\hat{A}-s_{13}^{2}-\alpha s_{12}^{2} c_{13}^{2}}{\cos 2 \theta_{13}^{m}}\right] .
\end{aligned}
$$

We consider the maximal value of $\theta_{23}\left(\theta_{23}=45^{\circ}\right)$ to derive the above expressions with non-zero $\delta_{\mathrm{CP}}$. From these expressions, one can get back the expressions derived in section 3 (eqs. (3.9) to (3.14)) for $\theta_{23}=45^{\circ}, \delta_{\mathrm{CP}}=0$ and retaining the terms which are of the order of $\alpha s_{13}$.

After the final rotation in $(1,2)$ block, the effective Hamiltonian is approximately diagonalized with two small non-zero off-diagonal elements which are of the order of $O\left(\Delta m_{31}^{2} \times \alpha^{2}\right)$ and $O\left(\Delta m_{31}^{2} \times \alpha s_{13}\right)$. Since these remaining off-diagonal terms are very small, we can safely neglect them. The modified mass-squared differences in the presence of non-zero $\delta_{\mathrm{CP}}$ can be expressed with the help of the following three diagonal elements

$$
\begin{aligned}
\frac{m_{3, m}^{2}}{2 E} & =\frac{\Delta_{31}}{2}\left[\lambda_{3}+\hat{A}+s_{13}^{2}+\alpha s_{12}^{2} c_{13}^{2}+\frac{\lambda_{3}-\hat{A}-s_{13}^{2}-\alpha s_{12}^{2} c_{13}^{2}}{\cos 2 \theta_{13}^{m}}\right], \\
\frac{m_{2, m}^{2}}{2 E} & =\frac{\Delta_{31}}{2}\left[\lambda_{1}+\lambda_{2}-\frac{\lambda_{1}-\lambda_{2}}{\cos 2 \theta_{12}^{m}}\right], \\
\frac{m_{1, m}^{2}}{2 E} & =\frac{\Delta_{31}}{2}\left[\lambda_{1}+\lambda_{2}+\frac{\lambda_{1}-\lambda_{2}}{\cos 2 \theta_{12}^{m}}\right] .
\end{aligned}
$$

In section 4 and section 5, we discuss in detail the evolution of three modified mixing angles and two mass-squared differences assuming $\delta_{\mathrm{CP}}=0$. Now, we show how the evolution of these mass-mixing parameters get affected in the presence of non-zero $\delta_{\mathrm{CP}}$. In figure 16, we show the evolution of three modified mixing angles as a function of neutrino energy for the baseline $1300 \mathrm{~km}$ for the SI case and considering one diagonal NSI parameter $\gamma$ and one off-diagonal NSI parameter $\varepsilon_{e \tau}$ with non-zero $\delta_{\mathrm{CP}}$. The black curve in each panel represents the SI case, which does not depend on $\delta_{\mathrm{CP}}$ as evident from eqs. (B.1) to (B.7), under the assumption that all the NSI parameters are zero. The red curve in each panel denotes the case with the NSI parameter $\gamma=0.1$ which is also almost independent of $\delta_{\mathrm{CP}}$ (see eqs. (B.1) to (B.7)). The evolution of $\theta_{23}^{m}$ does not depend on $\varepsilon_{e \tau}$ (see the extreme left panel of figure 16, where blue line completely overlap with black line) and it only affects the evolution of $\theta_{13}^{m}$ and $\theta_{12}^{m}$. The blue colored band in the middle panel is obtained by varying $\delta_{\mathrm{CP}}$ in the entire range of $-180^{\circ}$ to $180^{\circ}$ assuming $\varepsilon_{e \tau}=0.1$. Interestingly, near 

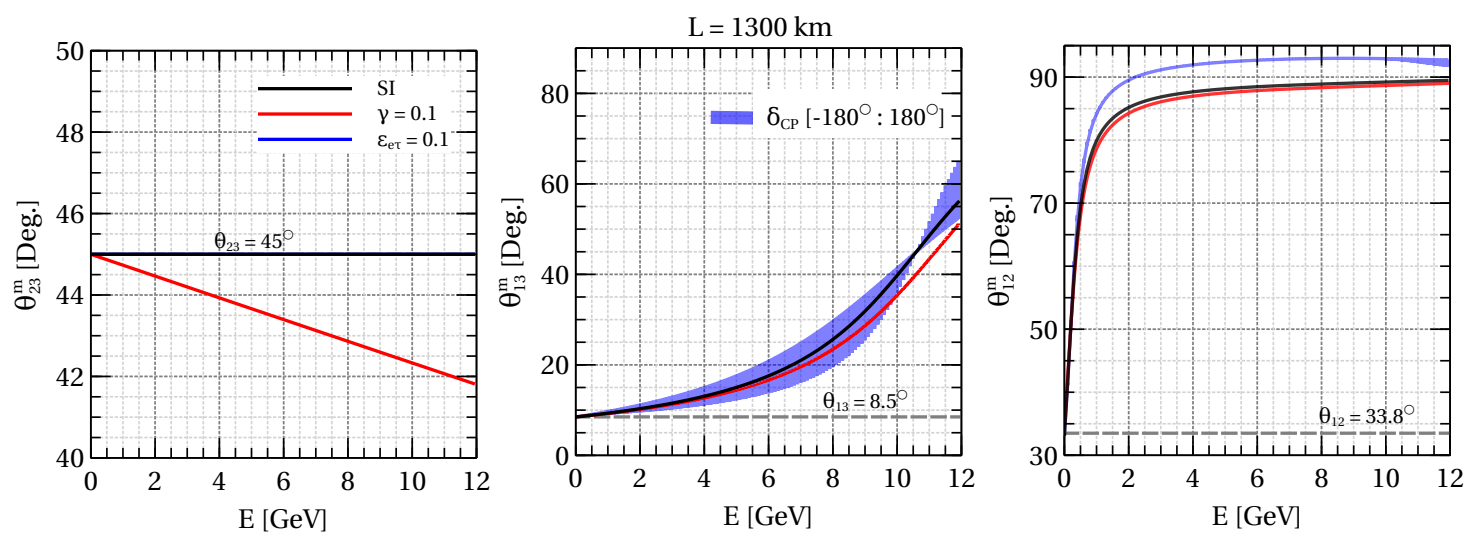

Figure 16. Evolution of three modified mixing angles $\theta_{23}^{m}$ (left panel), $\theta_{13}^{m}$ (middle panel), and $\theta_{12}^{m}$ (right panel) as a function of neutrino energy [see eqs. (B.1) to (B.7)] for the SI case (black curves) and SI+NSI cases considering the NSI parameters $\gamma$ (red curves) and $\varepsilon_{e \tau}$ (blue curves) with strength 0.1 for the baseline of $1300 \mathrm{~km}$. $\theta_{23}^{m}$ does not evolve in the SI case and with the NSI parameter $\varepsilon_{e \tau}$ (see the left panel where black and blue curves completely overlap with each other). In the middle and right panels, the blue colored bands are obtained by varying $\delta_{\mathrm{CP}}$ in the range of $-180^{\circ}$ to $180^{\circ}$ with $\varepsilon_{e \tau}=0.1$. Note that the evolution of $\theta_{23}^{m}$ does not depend on $\delta_{\mathrm{CP}}$ at all, whereas the evolution of $\theta_{13}^{m}$ and $\theta_{12}^{m}$ do not depend on $\delta_{\mathrm{CP}}$ for the SI case and with the diagonal NSI parameters $\gamma$. Values of the benchmark oscillation parameters in vacuum are taken from table 2 with $\theta_{23}=45^{\circ}$ assuming NMO.

$\theta_{13}$-resonance, the impact of $\delta_{\mathrm{CP}}$ vanishes as the denominator of the expression for $\theta_{13}^{m}$ becomes zero. The width of the blue color band due to the variation in $\delta_{\mathrm{CP}}$ is not significant in the case of $\theta_{12}^{m}$ evolution with $\varepsilon_{e \tau}=0.1$.

In figure 17, we show the evolution of two modified mass-squared differences $\Delta m_{31, m}^{2} \equiv$ $\left(m_{3, m}^{2}-m_{1, m}^{2}\right)$ and $\Delta m_{21, m}^{2} \equiv\left(m_{2, m}^{2}-m_{1, m}^{2}\right)$ as a function of neutrino energy (see eqs. (B.8) to (B.10)) for the SI case and considering one diagonal NSI parameter $\gamma$ and one off-diagonal NSI parameter $\varepsilon_{e \tau}$ for the baseline $1300 \mathrm{~km}$ with non-zero $\delta_{\mathrm{CP}}$. From figure 17, we observe that the evolution of $\Delta m_{31, m}^{2}$ (see left panel) do not depend on $\delta_{\mathrm{CP}}$ for the SI case and with the off-diagonal NSI parameter $\gamma$. Note that we observe similar features for these modified mixing angles in figure $16 . \delta_{\mathrm{CP}}$ only leave imprints in the evolution of $\Delta m_{31, m}^{2}$ and to some extent for $\Delta m_{21, m}^{2}$ when we consider the off-diagonal NSI parameter $\varepsilon_{e \tau}$.

In figure 18, we show a comparison between $\nu_{\mu} \rightarrow \nu_{e}$ appearance probabilities calculated using our approximate analytical expressions given in eqs. (B.1) to (B.10) (see dashed curves) and full numerical probabilities obtained from the publicly available software GLoBES (see solid curves). Here, we assume $L=1300 \mathrm{~km}, \theta_{23}=45^{\circ}$, normal mass ordering (NMO). The values of the other benchmark three-flavor oscillation parameters are taken from table 2 . We show a neck-to-neck comparison between numerical and analytical probabilities considering the off-diagonal NSI parameters $\varepsilon_{e \mu}$ (black lines) and $\varepsilon_{e \tau}$ (red lines) one-at-a-time with strength 0.1. Four different panels correspond to the four benchmark values of $\delta_{\mathrm{CP}}: 0,90^{\circ}$, $180^{\circ}$, and $-90^{\circ}$ (see legends). We observe that for all the four benchmark values of $\delta_{\mathrm{CP}}$, analytical oscillation probabilities obtained in this work have good agreement with the numerical oscillation probabilities. 

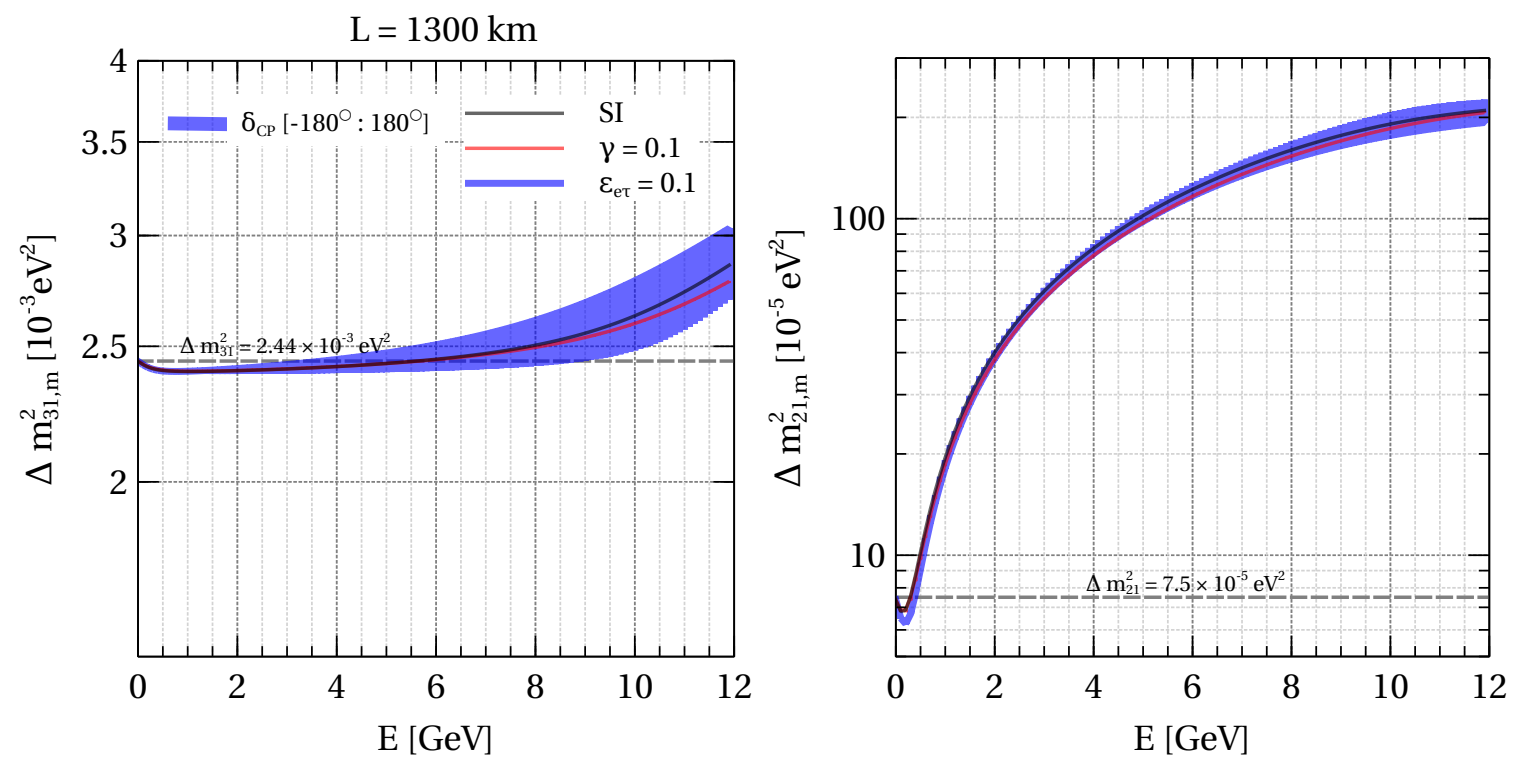

Figure 17. Evolution of two modified mass-squared differences $\Delta m_{31, m}^{2}$ (left panel) and $\Delta m_{21, m}^{2}$ (right panel) as a function of neutrino energy [see eqs. (B.8) to (B.10)] for the SI case (black curves) and SI+NSI cases considering the NSI parameters $\gamma$ (red curves) and $\varepsilon_{e \tau}$ (blue curves) with strength 0.1 for the baseline of $1300 \mathrm{~km}$. The blue colored bands are obtained by varying $\delta_{\mathrm{CP}}$ in the range of $-180^{\circ}$ to $180^{\circ}$ with $\varepsilon_{e \tau}=0.1$. Note that the evolution of $\Delta m_{31, m}^{2}$ and $\Delta m_{21, m}^{2}$ do not depend on $\delta_{\mathrm{CP}}$ for the SI case and with the diagonal NSI parameters $\gamma$. Values of the benchmark oscillation parameters in vacuum are taken from table 2 with $\theta_{23}=45^{\circ}$ assuming NMO.

\section{Calculation of the oscillation probabilities for neutrinos passing through various layers inside the Earth}

Matter density inside the Earth is not uniform and its value varies in the range of $2 \mathrm{~g} / \mathrm{cm}^{3}$ to $14 \mathrm{~g} / \mathrm{cm}^{3}$ as we move towards the center of Earth from the surface. It is also well-known that there is a sudden jumps in the Earth's matter densities at the boundaries of mantle-outer core and outer core-inner core (see figure 8). If a neutrino passes through only the crust or crust and mantle regions inside the Earth, then for those baselines, we can assume lineaveraged constant Earth matter densities to estimate the neutrino oscillation probabilities. When a neutrino penetrates the core (it happens for the baselines $L \gtrsim 10691 \mathrm{~km}$ ), then, for those baselines, the concept of line-averaged constant Earth matter density does not work since there is a sudden jumps in the matter density at the mantle-core boundary. In such cases, one needs to consider the exact PREM profile of the Earth as given in ref. [99].

In order to calculate the oscillation probabilities using our approximate analytical expressions for the modified mass-mixing parameters in matter, guided by the PREM profile of the Earth, we consider a simple four-layered profile consisting of crust, mantle, outer core, and inner core [100], which takes into account all the important characteristics of Earth. The radius and density of each layer of this four-layered profile are given in table 3 . To calculate the oscillation probability for a given baseline $L$, we first find out the length of the baseline which passes through each layer of this four-layered profile $\left(l_{x}\right.$ where $\left.x=1, \ldots 4\right)$. 

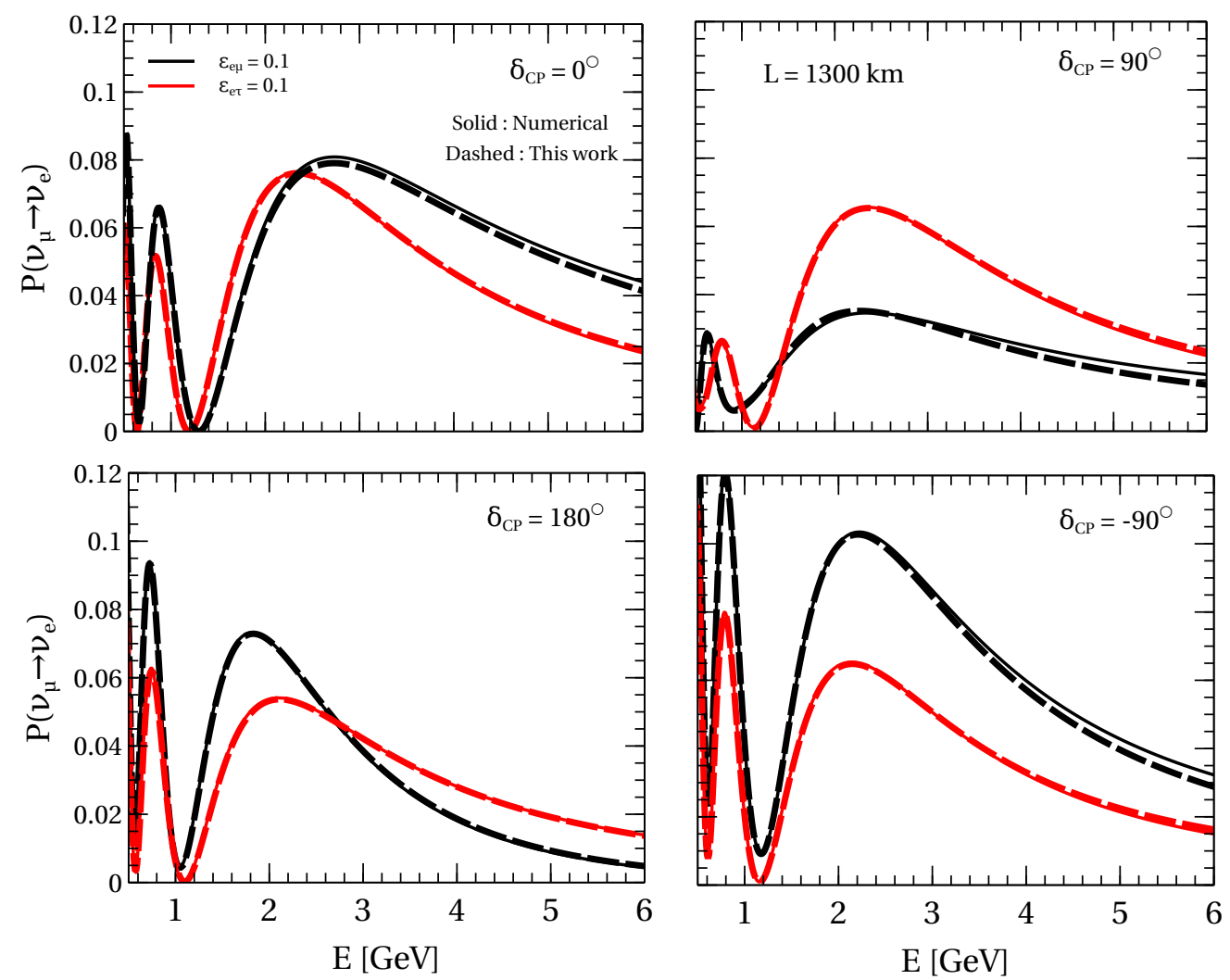

Figure 18. Comparison of $\nu_{\mu} \rightarrow \nu_{e}$ appearance probability estimated from our approximate analytical expressions (dashed curves), and the numerical calculations obtained from GLoBES (solid curves) in the presence of NC-NSI parameters $\varepsilon_{e \mu}$ (black curves) and $\varepsilon_{e \tau}$ (red curves) in the presence of non-zero $\delta_{\mathrm{CP}}$. We consider our benchmark values of $\delta_{\mathrm{CP}}: 0^{\circ}$ (upper left panel), $90^{\circ}$ (upper right panel), $180^{\circ}$ (lower left panel), and $-90^{\circ}$ (lower right panel). We assume $L=1300 \mathrm{~km}, \theta_{23}=45^{\circ}$, and NMO. The values of the other benchmark three-flavor oscillation parameters are taken from table 2 .

\begin{tabular}{|c|c|c|c|}
\hline Region & $\mathrm{R}_{\min }(\mathrm{km})$ & $\mathrm{R}_{\max }(\mathrm{km})$ & Average density $\left(\mathrm{g} / \mathrm{cm}^{3}\right)$ \\
\hline Inner core & 0 & 1220 & 13.0 \\
Outer core & 1220 & 3480 & 11.3 \\
Mantle & 3480 & 5701 & 5.0 \\
Crust & 5701 & 6371 & 3.3 \\
\hline
\end{tabular}

Table 3. The radius and average density of each layer of the four-layered profile of Earth which preserves all the important features of Earth. $R_{\min }\left(R_{\max }\right)$ is the radius at which a given layer starts (ends). 
Using the expressions of the modified mass-mixing parameters derived in this work, as given in eqs. (3.9) to (3.14) and eqs. (5.1) to (5.3), we calculate the probability amplitude matrix for each layer whose elements are given by the following expression ${ }^{15}[71]$

$$
A_{\beta \alpha}^{x}\left(L_{x}, \rho_{x}, E\right)=\sum_{j=1}^{3} U_{\beta j}\left(\rho_{x}\right) \exp \left(-i \frac{\Delta m_{j 1}^{2}\left(\rho_{x}\right)}{2 E} l_{x}\right) U_{\alpha j}^{*}\left(\rho_{x}\right) .
$$

To calculate the oscillation probability for a given baseline $L$, we first evaluate the resultant transition amplitude, which is the product of probability amplitude matrices estimated for various parts of the baseline passing through each layer. Then, we obtain the expression for the oscillation probability $\nu_{\alpha} \rightarrow \nu_{\beta}(\alpha, \beta=e, \mu, \tau)$ for a given baseline $L$ passing through the $n$ layers inside the Earth (where $n$ can be 1, 2, 3, 4)

$$
P\left(\nu_{\alpha} \rightarrow \nu_{\beta}, L\right)=\left|\prod_{x=1}^{n} A^{x}\left(L_{x}, \rho_{x}, E\right)\right|_{\beta \alpha}^{2},
$$

where $A^{x}\left(L_{x}, \rho_{x}, E\right)$ is the transition amplitude matrix for neutrino passing through the $x$-th layer. Following this prescription, for an example, $\nu_{\mu} \rightarrow \nu_{e}$ appearance probability for a baseline passing through all the four concentric layers inside the Earth can be written as

$$
P\left(\nu_{\mu} \rightarrow \nu_{e}, L\right)=\left|A^{1} A^{2} A^{3} A^{4} A^{3} A^{2} A^{1}\right|_{\mu e}^{2} .
$$

Similarly, one can calculate the $\nu_{\mu} \rightarrow \nu_{\mu}$ survival probability by squaring the $(\mu, \mu)$ element of the resultant probability amplitude matrix. We use this prescription to calculate the oscillation probabilities in our work (see figures 9, 10, and 13).

Open Access. This article is distributed under the terms of the Creative Commons Attribution License (CC-BY 4.0), which permits any use, distribution and reproduction in any medium, provided the original author(s) and source are credited.

\section{References}

[1] Particle Data Group collaboration, Review of Particle Physics, PTEP 2020 (2020) 083C01 [INSPIRE].

[2] Super-Kamiokande collaboration, Evidence for oscillation of atmospheric neutrinos, Phys. Rev. Lett. 81 (1998) 1562 [hep-ex/9807003] [INSPIRE].

[3] A. Marrone, Phenomenology of Three Neutrino Oscillations, talk given at The XIX International Workshop on Neutrino Telescopes, 18th to 26th February 2021, Padova, Italy [https://agenda.infn.it/event/24250/overview].

[4] NuFIT 5.0 (2020), http://www.nu-fit.org/.

\footnotetext{
${ }^{15} U\left(\rho_{x}\right)$ is estimated by replacing the vacuum oscillation parameters with their matter modified counterparts for each layer having path length $l_{x}$ and the corresponding density $\rho_{x}$ using eqs. (3.9) to (3.14). We calculate $\Delta m_{j 1}^{2}\left(\rho_{x}\right)$ for each layer having path length $l_{x}$ and the corresponding density $\rho_{x}$ using eqs. (5.1) to $(5.3)$.
} 
[5] I. Esteban, M.C. Gonzalez-Garcia, M. Maltoni, T. Schwetz and A. Zhou, The fate of hints: updated global analysis of three-flavor neutrino oscillations, JHEP 09 (2020) 178 [arXiv:2007.14792] [INSPIRE].

[6] P.F. de Salas et al., 2020 global reassessment of the neutrino oscillation picture, JHEP 02 (2021) 071 [arXiv:2006.11237] [INSPIRE].

[7] R.N. Mohapatra et al., Theory of neutrinos: A White paper, Rept. Prog. Phys. 70 (2007) 1757 [hep-ph/0510213] [INSPIRE].

[8] A. Strumia and F. Vissani, Neutrino masses and mixings and..., hep-ph/0606054 [INSPIRE].

[9] M.C. Gonzalez-Garcia and M. Maltoni, Phenomenology with Massive Neutrinos, Phys. Rept. 460 (2008) 1 [arXiv:0704.1800] [INSPIRE].

[10] SUPER-KAMIOKANDE collaboration, Evidence for an oscillatory signature in atmospheric neutrino oscillation, Phys. Rev. Lett. 93 (2004) 101801 [hep-ex/0404034] [INSPIRE].

[11] ICECUBE collaboration, Measurement of Atmospheric Neutrino Oscillations at 6-56 GeV with IceCube DeepCore, Phys. Rev. Lett. 120 (2018) 071801 [arXiv:1707.07081] [INSPIRE].

[12] ANTARES collaboration, Measuring the atmospheric neutrino oscillation parameters and constraining the 3+1 neutrino model with ten years of ANTARES data, JHEP 06 (2019) 113 [arXiv: 1812.08650] [INSPIRE].

[13] DAYA BAY collaboration, Measurement of the Electron Antineutrino Oscillation with 1958 Days of Operation at Daya Bay, Phys. Rev. Lett. 121 (2018) 241805 [arXiv: 1809.02261] [INSPIRE].

[14] RENO collaboration, Observation of Reactor Electron Antineutrino Disappearance in the RENO Experiment, Phys. Rev. Lett. 108 (2012) 191802 [arXiv:1204.0626] [INSPIRE].

[15] T2K collaboration, Constraint on the matter-antimatter symmetry-violating phase in neutrino oscillations, Nature 580 (2020) 339 [Erratum ibid. 583 (2020) E16] [arXiv:1910.03887] [INSPIRE].

[16] T2K collaboration, Improved constraints on neutrino mixing from the T2K experiment with $3.13 \times 10^{21}$ protons on target, Phys. Rev. D 103 (2021) 112008 [arXiv:2101.03779] [INSPIRE].

[17] NOvA collaboration, First Measurement of Neutrino Oscillation Parameters using Neutrinos and Antineutrinos by NOvA, Phys. Rev. Lett. 123 (2019) 151803 [arXiv: 1906.04907] [INSPIRE].

[18] DUNE collaboration, Deep Underground Neutrino Experiment (DUNE), Far Detector Technical Design Report, Volume II: DUNE Physics, arXiv:2002.03005 [INSPIRE].

[19] DUNE collaboration, Experiment Simulation Configurations Approximating DUNE TDR, arXiv:2103.04797 [INSPIRE].

[20] Hyper-Kamiokande Proto- collaboration, Physics potential of a long-baseline neutrino oscillation experiment using a J-PARC neutrino beam and Hyper-Kamiokande, PTEP 2015 (2015) 053C02 [arXiv: 1502.05199] [INSPIRE].

[21] HyPER-KAmiOKANDE collaboration, Physics potentials with the second Hyper-Kamiokande detector in Korea, PTEP 2018 (2018) 063C01 [arXiv:1611.06118] [INSPIRE].

[22] ESSNuSB collaboration, A very intense neutrino super beam experiment for leptonic CP-violation discovery based on the European spallation source linac, Nucl. Phys. B $\mathbf{8 8 5}$ (2014) 127 [arXiv:1309.7022] [INSPIRE]. 
[23] M.M. Devi, T. Thakore, S.K. Agarwalla and A. Dighe, Enhancing sensitivity to neutrino parameters at INO combining muon and hadron information, JHEP 10 (2014) 189 [arXiv: 1406.3689] [INSPIRE].

[24] ICAL collaboration, Physics Potential of the ICAL detector at the India-based Neutrino Observatory (INO), Pramana 88 (2017) 79 [arXiv:1505.07380] [INSPIRE].

[25] A. Kumar, A. Khatun, S.K. Agarwalla and A. Dighe, From oscillation dip to oscillation valley in atmospheric neutrino experiments, Eur. Phys. J. C 81 (2021) 190 [arXiv: 2006. 14529] [INSPIRE].

[26] JUNO collaboration, Neutrino Physics with JUNO, J. Phys. G 43 (2016) 030401 [arXiv: 1507.05613] [INSPIRE].

[27] TheIA collaboration, THEIA: an advanced optical neutrino detector, Eur. Phys. J. C 80 (2020) 416 [arXiv: 1911.03501] [INSPIRE].

[28] C.A. Argüelles et al., New opportunities at the next-generation neutrino experiments I: BSM neutrino physics and dark matter, Rept. Prog. Phys. 83 (2020) 124201 [arXiv:1907.08311] [INSPIRE].

[29] S.K. Agarwalla, BSM Searches in Neutrino Experiments, talk given at The XXIX International Conference on Neutrino Physics and Astrophysics (Neutrino 2020), 22nd June to 2nd July 2020, Fermilab, Chicago, U.S.A. [https://conferences.fnal.gov/nu2020/].

[30] L. Wolfenstein, Neutrino Oscillations in Matter, Phys. Rev. D 17 (1978) 2369 [InSPIRE].

[31] J.W.F. Valle, Resonant Oscillations of Massless Neutrinos in Matter, Phys. Lett. B 199 (1987) 432 [INSPIRE].

[32] M.M. Guzzo, A. Masiero and S.T. Petcov, On the MSW effect with massless neutrinos and no mixing in the vacuum, Phys. Lett. B 260 (1991) 154 [INSPIRE].

[33] E. Roulet, MSW effect with flavor changing neutrino interactions, Phys. Rev. D 44 (1991) R935 [INSPIRE].

[34] Y. Grossman, Nonstandard neutrino interactions and neutrino oscillation experiments, Phys. Lett. B 359 (1995) 141 [hep-ph/9507344] [INSPIRE].

[35] M.M. Guzzo, H. Nunokawa, P.C. de Holanda and O.L.G. Peres, On the massless 'just-so' solution to the solar neutrino problem, Phys. Rev. D 64 (2001) 097301 [hep-ph/0012089] [INSPIRE].

[36] P. Huber and J.W.F. Valle, Nonstandard interactions: Atmospheric versus neutrino factory experiments, Phys. Lett. B 523 (2001) 151 [hep-ph/0108193] [INSPIRE].

[37] A.M. Gago et al., Global analysis of the postSNO solar neutrino data for standard and nonstandard oscillation mechanisms, Phys. Rev. D 65 (2002) 073012 [hep-ph/0112060] [INSPIRE].

[38] F.J. Escrihuela, M. Tortola, J.W.F. Valle and O.G. Miranda, Global constraints on muon-neutrino non-standard interactions, Phys. Rev. D 83 (2011) 093002 [arXiv:1103.1366] [INSPIRE].

[39] M.C. Gonzalez-Garcia, M. Maltoni and J. Salvado, Testing matter effects in propagation of atmospheric and long-baseline neutrinos, JHEP 05 (2011) 075 [arXiv:1103.4365] [INSPIRE].

[40] T. Ohlsson, Status of non-standard neutrino interactions, Rept. Prog. Phys. 76 (2013) 044201 [arXiv:1209.2710] [INSPIRE]. 
[41] M.C. Gonzalez-Garcia and M. Maltoni, Determination of matter potential from global analysis of neutrino oscillation data, JHEP 09 (2013) 152 [arXiv:1307.3092] [INSPIRE].

[42] O.G. Miranda and H. Nunokawa, Non standard neutrino interactions: current status and future prospects, New J. Phys. 17 (2015) 095002 [arXiv:1505.06254] [INSPIRE].

[43] Y. Farzan and M. Tortola, Neutrino oscillations and Non-Standard Interactions, Front. in Phys. 6 (2018) 10 [arXiv:1710.09360] [INSPIRE].

[44] A. Khatun, S.S. Chatterjee, T. Thakore and S. Kumar Agarwalla, Enhancing sensitivity to non-standard neutrino interactions at INO combining muon and hadron information, Eur. Phys. J. C 80 (2020) 533 [arXiv:1907.02027] [INSPIRE].

[45] P.S. Bhupal Dev et al., Neutrino Non-Standard Interactions: A Status Report, SciPost Phys. Proc. 2 (2019) 001 [INSPIRE].

[46] A. Kumar, A. Khatun, S.K. Agarwalla and A. Dighe, A New Approach to Probe Non-Standard Interactions in Atmospheric Neutrino Experiments, JHEP 04 (2021) 159 [arXiv: 2101.02607] [INSPIRE].

[47] S.P. Mikheyev and A.Y. Smirnov, Resonance Amplification of Oscillations in Matter and Spectroscopy of Solar Neutrinos, Sov. J. Nucl. Phys. 42 (1985) 913 [inSPIRE].

[48] S.P. Mikheev and A.Y. Smirnov, Resonant amplification of neutrino oscillations in matter and solar neutrino spectroscopy, Nuovo Cim. C 9 (1986) 17 [INSPIRE].

[49] G. Barenboim, P.B. Denton, S.J. Parke and C.A. Ternes, Neutrino Oscillation Probabilities through the Looking Glass, Phys. Lett. B 791 (2019) 351 [arXiv: 1902.00517] [INSPIRE].

[50] S.T. Petcov and S. Toshev, Three Neutrino Oscillations in Matter: Analytical Results in the Adiabatic Approximation, Phys. Lett. B 187 (1987) 120 [INSPIRE].

[51] C.W. Kim and W.K. Sze, Adiabatic Resonant Oscillations of Solar Neutrinos in Three Generations, Phys. Rev. D 35 (1987) 1404 [INSPIRE].

[52] J. Arafune and J. Sato, CP and T violation test in neutrino oscillation, Phys. Rev. D 55 (1997) 1653 [hep-ph/9607437] [INSPIRE].

[53] J. Arafune, M. Koike and J. Sato, CP violation and matter effect in long baseline neutrino oscillation experiments, Phys. Rev. D 56 (1997) 3093 [Erratum ibid. 60 (1999) 119905] [hep-ph/9703351] [INSPIRE].

[54] T. Ohlsson and H. Snellman, Three flavor neutrino oscillations in matter, J. Math. Phys. 41 (2000) 2768 [Erratum ibid. 42 (2001) 2345] [hep-ph/9910546] [INSPIRE].

[55] M. Freund, Analytic approximations for three neutrino oscillation parameters and probabilities in matter, Phys. Rev. D 64 (2001) 053003 [hep-ph/0103300] [INSPIRE].

[56] A. Cervera et al., Golden measurements at a neutrino factory, Nucl. Phys. B 579 (2000) 17 [Erratum ibid. 593 (2001) 731] [hep-ph/0002108] [INSPIRE].

[57] E.K. Akhmedov, R. Johansson, M. Lindner, T. Ohlsson and T. Schwetz, Series expansions for three flavor neutrino oscillation probabilities in matter, JHEP 04 (2004) 078 [hep-ph/0402175] [INSPIRE].

[58] K. Asano and H. Minakata, Large $\theta_{13}$ Perturbation Theory of Neutrino Oscillation for Long-Baseline Experiments, JHEP 06 (2011) 022 [arXiv: 1103.4387] [INSPIRE].

[59] S.K. Agarwalla, Y. Kao and T. Takeuchi, Analytical approximation of the neutrino oscillation matter effects at large $\theta_{13}$, JHEP 04 (2014) 047 [arXiv: 1302.6773] [INSPIRE]. 
[60] H. Minakata and S.J. Parke, Simple and Compact Expressions for Neutrino Oscillation Probabilities in Matter, JHEP 01 (2016) 180 [arXiv: 1505.01826] [INSPIRE].

[61] P.B. Denton, H. Minakata and S.J. Parke, Compact Perturbative Expressions For Neutrino Oscillations in Matter, JHEP 06 (2016) 051 [arXiv: 1604.08167] [INSPIRE].

[62] M.C. Gonzalez-Garcia, Y. Grossman, A. Gusso and Y. Nir, New CP-violation in neutrino oscillations, Phys. Rev. D 64 (2001) 096006 [hep-ph/0105159] [INSPIRE].

[63] T. Ota, J. Sato and N.-a. Yamashita, Oscillation enhanced search for new interaction with neutrinos, Phys. Rev. D 65 (2002) 093015 [hep-ph/0112329] [INSPIRE].

[64] O. Yasuda, On the exact formula for neutrino oscillation probability by Kimura, Takamura and Yokomakura, arXiv:0704.1531 [INSPIRE].

[65] J. Kopp, M. Lindner, T. Ota and J. Sato, Non-standard neutrino interactions in reactor and superbeam experiments, Phys. Rev. D 77 (2008) 013007 [arXiv: 0708.0152] [InSPIRE].

[66] N.C. Ribeiro, H. Minakata, H. Nunokawa, S. Uchinami and R. Zukanovich-Funchal, Probing Non-Standard Neutrino Interactions with Neutrino Factories, JHEP 12 (2007) 002 [arXiv:0709.1980] [INSPIRE].

[67] M. Blennow and T. Ohlsson, Approximative two-flavor framework for neutrino oscillations with non-standard interactions, Phys. Rev. D 78 (2008) 093002 [arXiv:0805.2301] [INSPIRE].

[68] T. Kikuchi, H. Minakata and S. Uchinami, Perturbation Theory of Neutrino Oscillation with Nonstandard Neutrino Interactions, JHEP 03 (2009) 114 [arXiv: 0809.3312] [INSPIRE].

[69] D. Meloni, T. Ohlsson and H. Zhang, Exact and Approximate Formulas for Neutrino Mixing and Oscillations with Non-Standard Interactions, JHEP 04 (2009) 033 [arXiv:0901.1784] [INSPIRE].

[70] S.K. Agarwalla, Y. Kao, D. Saha and T. Takeuchi, Running of Oscillation Parameters in Matter with Flavor-Diagonal Non-Standard Interactions of the Neutrino, JHEP 11 (2015) 035 [arXiv: 1506.08464] [INSPIRE].

[71] V.D. Barger, K. Whisnant, S. Pakvasa and R.J.N. Phillips, Matter Effects on Three-Neutrino Oscillations, Phys. Rev. D 22 (1980) 2718 [inSPIRE].

[72] H.W. Zaglauer and K.H. Schwarzer, The Mixing Angles in Matter for Three Generations of Neutrinos and the MSW Mechanism, Z. Phys. C 40 (1988) 273 [INSPIRE].

[73] T. Ohlsson and H. Snellman, Neutrino oscillations with three flavors in matter: Applications to neutrinos traversing the Earth, Phys. Lett. B $\mathbf{4 7 4}$ (2000) 153 [Erratum ibid. 480 (2000) 419] [hep-ph/9912295] [INSPIRE].

[74] K. Kimura, A. Takamura and H. Yokomakura, Exact formula of probability and CP-violation for neutrino oscillations in matter, Phys. Lett. B 537 (2002) 86 [hep-ph/0203099] [INSPIRE].

[75] K. Kimura, A. Takamura and H. Yokomakura, Exact formulas and simple CP dependence of neutrino oscillation probabilities in matter with constant density, Phys. Rev. D 66 (2002) 073005 [hep-ph/0205295] [INSPIRE].

[76] Z.-z. Xing, S. Zhou and Y.-L. Zhou, Renormalization-Group Equations of Neutrino Masses and Flavor Mixing Parameters in Matter, JHEP 05 (2018) 015 [arXiv:1802.00990] [INSPIRE].

[77] X. Wang and S. Zhou, Analytical solutions to renormalization-group equations of effective neutrino masses and mixing parameters in matter, JHEP 05 (2019) 035 [arXiv:1901.10882] [INSPIRE]. 
[78] X. Wang and S. Zhou, On the Properties of the Effective Jarlskog Invariant for Three-flavor Neutrino Oscillations in Matter, Nucl. Phys. B 950 (2020) 114867 [arXiv:1908.07304] [INSPIRE].

[79] C. Jarlskog, Commutator of the Quark Mass Matrices in the Standard Electroweak Model and a Measure of Maximal CP-violation, Phys. Rev. Lett. 55 (1985) 1039 [INSPIRE].

[80] V.A. Naumov, Three neutrino oscillations in matter, CP-violation and topological phases, Int. J. Mod. Phys. D 1 (1992) 379 [INSPIRE].

[81] P.F. Harrison and W.G. Scott, $C P$ and T violation in neutrino oscillations and invariance of Jarlskog's determinant to matter effects, Phys. Lett. B 476 (2000) 349 [hep-ph/9912435] [INSPIRE].

[82] C.G.J. Jacobi, Über ein leichtes Verfahren, die in der Theorie der Säkularstörangen vorkommenden Gleichungen numerisch aufzuloösen, Crelle 30 (1846) 51.

[83] L. Wolfenstein, Neutrino Oscillations and Stellar Collapse, Phys. Rev. D 20 (1979) 2634 [INSPIRE].

[84] S.-F. Ge and S.J. Parke, Scalar Nonstandard Interactions in Neutrino Oscillation, Phys. Rev. Lett. 122 (2019) 211801 [arXiv:1812.08376] [INSPIRE].

[85] D. Aristizabal Sierra, V. De Romeri and N. Rojas, COHERENT analysis of neutrino generalized interactions, Phys. Rev. D 98 (2018) 075018 [arXiv: 1806. 07424] [INSPIRE].

[86] J. Heeck and W. Rodejohann, Gauged $L_{\mu}-L_{\tau}$ Symmetry at the Electroweak Scale, Phys. Rev. D 84 (2011) 075007 [arXiv:1107.5238] [INSPIRE].

[87] Y. Farzan and I.M. Shoemaker, Lepton Flavor Violating Non-Standard Interactions via Light Mediators, JHEP 07 (2016) 033 [arXiv: 1512.09147] [INSPIRE].

[88] Y. Farzan and J. Heeck, Neutrinophilic nonstandard interactions, Phys. Rev. D 94 (2016) 053010 [arXiv: 1607.07616] [INSPIRE].

[89] K.S. Babu, A. Friedland, P.A.N. Machado and I. Mocioiu, Flavor Gauge Models Below the Fermi Scale, JHEP 12 (2017) 096 [arXiv:1705.01822] [INSPIRE].

[90] M.B. Wise and Y. Zhang, Lepton Flavorful Fifth Force and Depth-dependent Neutrino Matter Interactions, JHEP 06 (2018) 053 [arXiv: 1803.00591] [INSPIRE].

[91] H. Minakata, Probing Non-Standard Neutrino Physics at Neutrino Factory and T2KK, in 4th International Workshop on Neutrino Oscillations in Venice: Ten Years after the Neutrino Oscillations, pp. 361-380 (2008) [arXiv:0805.2435] [INSPIRE].

[92] B. Pontecorvo, Inverse beta processes and nonconservation of lepton charge, Sov. Phys. JETP 7 (1958) 172 [INSPIRE].

[93] Z. Maki, M. Nakagawa and S. Sakata, Remarks on the unified model of elementary particles, Prog. Theor. Phys. 28 (1962) 870 [INSPIRE].

[94] B. Pontecorvo, Neutrino Experiments and the Problem of Conservation of Leptonic Charge, Sov. Phys. JETP 26 (1968) 984 [InSPIRE].

[95] I. Esteban, M.C. Gonzalez-Garcia, M. Maltoni, I. Martinez-Soler and J. Salvado, Updated constraints on non-standard interactions from global analysis of oscillation data, JHEP 08 (2018) 180 [Addendum ibid. 12 (2020) 152] [arXiv: 1805.04530] [INSPIRE].

[96] S.S. Chatterjee, A. Dasgupta and S.K. Agarwalla, Exploring Flavor-Dependent Long-Range Forces in Long-Baseline Neutrino Oscillation Experiments, JHEP 12 (2015) 167 [arXiv: 1509.03517] [INSPIRE]. 
[97] P. Huber, M. Lindner and W. Winter, Simulation of long-baseline neutrino oscillation experiments with GLoBES (General Long Baseline Experiment Simulator), Comput. Phys. Commun. 167 (2005) 195 [hep-ph/0407333] [INSPIRE].

[98] P. Huber, J. Kopp, M. Lindner, M. Rolinec and W. Winter, New features in the simulation of neutrino oscillation experiments with GLoBES 3.0: General Long Baseline Experiment Simulator, Comput. Phys. Commun. 177 (2007) 432 [hep-ph/0701187] [InSPIRE].

[99] A.M. Dziewonski and D.L. Anderson, Preliminary reference earth model, Phys. Earth Planet. Interiors 25 (1981) 297.

[100] Super-Kamiokande collaboration, Atmospheric neutrino oscillation analysis with external constraints in Super-Kamiokande I-IV, Phys. Rev. D 97 (2018) 072001 [arXiv:1710.09126] [INSPIRE].

[101] M.C. Banuls, G. Barenboim and J. Bernabeu, Medium effects for terrestrial and atmospheric neutrino oscillations, Phys. Lett. B 513 (2001) 391 [hep-ph/0102184] [INSPIRE].

[102] R. Gandhi, P. Ghoshal, S. Goswami, P. Mehta and S.U. Sankar, Large matter effects in $\nu_{\mu} \rightarrow \nu_{\tau}$ oscillations, Phys. Rev. Lett. 94 (2005) 051801 [hep-ph/0408361] [INSPIRE].

[103] R. Gandhi, P. Ghoshal, S. Goswami, P. Mehta and S.U. Sankar, Earth matter effects at very long baselines and the neutrino mass hierarchy, Phys. Rev. D 73 (2006) 053001 [hep-ph/0411252] [INSPIRE].

[104] J. Kopp and M. Lindner, Detecting atmospheric neutrino oscillations in the ATLAS detector at CERN, Phys. Rev. D 76 (2007) 093003 [arXiv:0705.2595] [INSPIRE].

[105] I. Mocioiu and W. Wright, Non-standard neutrino interactions in the $\mu-\tau$ sector, Nucl. Phys. B 893 (2015) 376 [arXiv:1410.6193] [INSPIRE].

[106] M.C. Gonzalez-Garcia and M. Maltoni, Atmospheric neutrino oscillations and new physics, Phys. Rev. D 70 (2004) 033010 [hep-ph/0404085] [InSPIRE].

[107] F. Capozzi, E. Di Valentino, E. Lisi, A. Marrone, A. Melchiorri and A. Palazzo, Unfinished fabric of the three neutrino paradigm, Phys. Rev. D 104 (2021) 083031 [arXiv:2107.00532] [INSPIRE]. 